\title{
CORRIGENDA
}

p.8 11.15-16. Delete "oither...or".

p.9 1.3. For "be determinate...meaning", read "have - forr and a meanine".

p.92 1.3. For "determinate", read "specifio".

p.130 11.16-17. For "the combination of the adjective", read "the combination of one of tine adjectives".

p.140, note 1. Fox "p.27", read "pp.23-27".

p.189, note 3. For "p.120", read "p.210".

p.201, 1.19. "Iakoff" = "Robin Lakoff", hence read "Bhe' for "hen throuchout section 4.251 .

p.214. 21.10-11. Por "equivalence in the force of epeecl ects", reed "equivelence of 1110cutionary coto".

p.214, note 1. For "On the force of speech neten", reed "On 1110cutionary e.cte".

D.215. 1.9. Por "locutionasy", read "Illocutionaxy", an delete "In torce".

p.217. 1.20. Delete "the force of".

p.261, 1.3. Fox "with the same foxce In speech acts", read "as equivalent $11100 u t 10$ nery cete".

p.261, 1.6. For "with difforeut force in speoch ecte", read "In nonequivalent 1llocutionsry sets".

.p.262, 1.16. A Iine has been onittod. Reads "Primatolo Is prinetolosw. 0.50 the 11locutionary act of expleiaing to $X$ las distinat from the perloc utionary act or isetting $\mathbf{X}$ to underatend) what prineto.10 EJ la..." 
ProQuest Number: 10672745

All rights reserved

INFORMATION TO ALL USERS

The quality of this reproduction is dependent upon the quality of the copy submitted.

In the unlikely event that the author did not send a complete manuscript and there are missing pages, these will be noted. Also, if material had to be removed, a note will indicate the deletion.

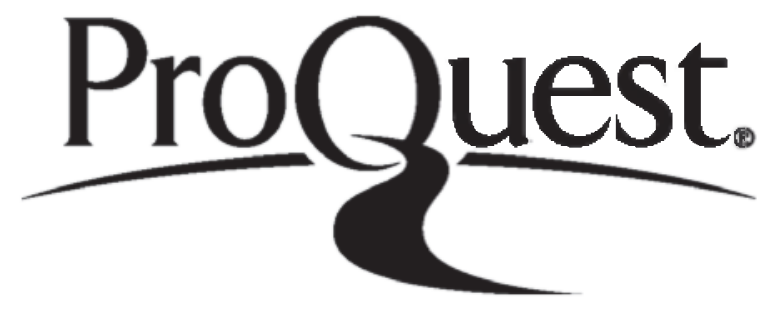

ProQuest 10672745

Published by ProQuest LLC (2017). Copyright of the Dissertation is held by the Author.

All rights reserved.

This work is protected against unauthorized copying under Title 17, United States Code Microform Edition (C) ProQuest LLC.

ProQuest LLC.

789 East Eisenhower Parkway

P.O. Box 1346

Ann Arbor, Ml 48106-1346 
SYNONYMY AND IINGUISTIC ANALYSIS

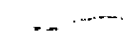

Roy Harris

Thesis submitted to the University of Iondon for the degree of Ph.D.

1970

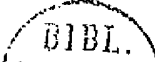


ABSTRACT

A synonymity statement presents a comparison of linguistic items in respect of their linguistic meanings, abstracting from the particular meanings in question and giving simply an assessment of 'semantic sameness' vs. 'semantic difference'. Questions connected with synonymity statements in linguistic analysis are considered in the present study under the following heads: (i) the justification of synonymity statements in terms of the postulates of linguistic analysis adopted; ( $i i)$ the role of synonymity statements in phonology; (iii) the role of synonymity statements in grammar; (iv) the role of synonymity statements in semantic analysis; and $(v)$ the justification of synonymity statements in terms of a concept of 'linguistic knowledge'. In each case, according as the synonymity statement functions as explicans or explicandum, the theoretical positions generally adopted are examined, and arguments for or against are analysed. The ultimate objective in view is to establish a viable basis for integrating a linguistic analysis which employs synonymity statements with a theory of speech acts which will account for communication between languageusers. 
TABLE OF CONTENTS

Introduction

Page

1

Ch.1 Synonymy, form and meaning

Ch.2 Synonymy and phonological analysis

Ch.4 Synonymy and semantic analysis

Ch.5 Synonymy and Iinguistic knowledge

Bibliography

276 
Introduction 
An attempt is made in the present study to survey the whole range of problems connected with developing an account of synonymy satisfactory for descriptive Iinguistic analysis. The main purpose of such an account will be the justification of the use of 'synonymity statements ${ }^{1}$ in linguistic analysis.

Synonymity statements may be characterized as statements cast in or reducible to the form

'a and $\underline{b}$ are synonymous in $\underline{L}$

or 'aㅡ and $\underline{b}$ are not synonymous in $\underline{\underline{I}}$ ',

where $I_{\text {is }}$ ise particular language under description and a and $\underline{b}$ are expressions ${ }^{2}$ of that language.

1. These statements formulate what are called 'synonymity hypotheses' in the terminology proposed by Naess (Naess 1953). The status of the hypotheses is comparable to that of hypotheses in other empirical sciences: they 'may be said to make claims about actual or possible interpretative processes in the same way as hypotheses about certain substances being explosives make claims about actual or possible explosions' (loc.cit. p. 17). A hypothesis of this kind may, of course, be involved in certain explanations without being overtly formulated: it would then be necessary, strictly, to speak of the use of a 'synonymity hypothesis' rather than the use of a 'synonymity statement', but for present purposes the distinction is of no consequence.

2. The term synonym is traditionally restricted to items of the kind which appear as lemmata in conventional dictionaries, chiefly common nouns, verbs, adjectives and adverbs. This usage goes back to the definition given in Aristotle's Rhetoric III, 2, 1404b, and is continued by the eighteenth-and nineteenth-century synonymists who compiled synonym dictionaries of the modern European languages. But it has been generally recognized more recently that 'synonymity occurs not only in the case of single words, but both above and below words' (Collinson 1939). In current discussions of the subject, 'synonymous' expressions may include words, bound morphs, phrases, clauses, sentences and sequences of sentences. We do not have to commit ourselves as to the exact grammatical status of the expressions asserted - or denied - to be synonymous (unless it is particularly relevant to do so). 
The questions arising with respect to synonymity statements fall into two groups and may be summarized as follows.

(1) What is the theoretical basis of a position which allows the possibility that natural languages may have words, sentences, etc. that differ in form but not in meaning?

Topics falling under this head are discussed in Ch. $I$.

(2) What particular purposes are synonymity statements called upon to serve in linguistic analysis, and what is their explanatory function?

Under (2) it is necessary to distinguish between the use of synonymity statements (i) in phonological analysis (in connexion with procedures for determining phonemes); (ii) in grammatical analysis (in connexion with the establishment of certain grammatical relations); and (iii) in semantic analysis (in connexion with certain features of semantic descriptions).

These three cases are considered separately in Chs. 2, 3, and 4.

In conclusion, the role of synonymity statements in relation to a theory of 'linguistic knowledge' is discussed in Ch. 5.

Attention is focussed throughout upon arguments and evidence which contribute to establishing the basic requirements of an account of synonymy, and topics have been included or omitted accordingly. A brief comments on two points may serve to indicate the scope of the inquiry.

1. We shall not be concerned with the verification of 'linguistic 
characterizations' in the sense proposed by Searle. Searle claims: 'As a native speaker of English, I know that "oculist" is exactly synonymous with "eye doctor"I, and further that 'the claim that "oculist" means eye doctor is not a claim that has to satisfy any criteria which philosophers might propose for synonymy, but rather any proposed criterion for synonymy has to be consistent with such facts as that "eye doctor" is synonymous with "oculist",". This standpoint is quite different from that taken in the present study. From our standpoint there is little or no interest in Searle's claim to know, qua native speaker of Inglish, that certain expressions 'are synonymous'. It might perhaps be urged that if eye doctor and oculist are in fact synonymous in English, then Searle is right so to claim. But if that is the case, the claim might just as well be made by a Martian; i.e. if we are interested in whether or not the claim is right, it does not matter who makes it or how he knows it. More to the point would be to discover what Searle thinks he is claiming when he claims that one expression is 'exactly synonymous' with another. But that also would be of merely subsidiary interest. Our concern, rather, is with the elucidation of what a linguist would be explaining if he attempted to account for certain observable facts about Searle's linguistic behaviour

1. Searle, 1969 p. 11.

2. Searle, 1969 p. 9. 
(and that of other speakers of English) by postulating the synonymity of oculist and eye doctor as part of Searle's (and others') linguistic knowledge.

2. By the same token, such matters as proposals concerning distributional criteria for synonymity, or the 'quantification' of synonymy, are of no interest here. In connexion with the former, Chomsky observes that 'many linguists have proposed that synonymy be somehow measured in terms of degree of distributional similarity (cf. e.g. H. M. Honigswald Language Change and Linguistic Reconstruction, Chicago, 1960; H. Frei 'Désaccords' Cahiers Ferdinand de Saussure 18.35-51, 1961) and have then concluded that such pairs as 'bachelor' and 'unmarried man' are not synonymous, since one, but not the other, can occur in the context hood etc... But all that this observation shows is that the proposed criterion is entirely wrong, as, indeed, it clearly is. "I The issue between Chomsky and the distributionalist is irresolvable in the sense that if the distributionalist is right then Chomsky's counterexample - or any other - must be mistaken. On the other hand, if the distributionalist thesis can be confirmed or refuted on the basis of examples or counterexamples, then it is both possible and necessary to establish synonymity independently of the distributional evidence. But the assumption that meanings determine distribution in such a way that difference in distribution is a

1. Chomsky 1962 p. 527. 
sufficient condition of difference in meaning simply leaves one puzzled as to what concept of 'meaning' is being invoked. It seems that what we have here is what amounts to equation of 'meaning' with, 'distribution', in which case the proposals concerning synonymy are trivial.

As regards 'quantification' of synonymy, it may be pointed out that drawing distinctions between various degrees of or approximations to synonymity on the basis of 'componential analysis' or 'semantic classification' (cf. Sparck Jones 1964) is in all essentials a continuation of the approach of the synonymists. But this approach presupposes rather than proposes answers to the questions we are trying to answer. It is evident that, given a system of semantic categorization, synonymous expressions will be those receiving identical characterizations under that system. Other degrees or varieties of synonymity can be duly distinguished if we wish. Weinreich proposes to call any pair of terms $\underline{A}$ and $\underline{A}$ ' 'immediate synonyms' if their designata differ by one component. ${ }^{6}$ Ducháček proposes a distinction between 'perfect synonyms', 'approximate synonyms', and 'words semantically related'; but concedes that there is room for disagreement over the criteria to be applied.' Abraham and Kiefer propose a distinction between 'full' and 'less-than-full' synonymy, with reference to tree diagrams representing the various grammatical and semantic categories of a word.

6. Weinreich $1963 \oint 4.2$.

7. Ducháček 1964. 
According to their definition, 'between two words, $w_{1}$ and $w_{2}$, a full synonymy holds if, and only if, their trees have exactly the same branching structure (i.e. the same paths) and exactly the same labels on the corresponding nodes', while 'an i-ways synonymy holds if, and only if, they have in their tree graphs $i$ paths in common'. The various paths distinguish between different uses of the word in question; but if we ask how these uses are determined, all we are told is that they can be found 'recorded in any good explanatory dictionary ${ }^{8}$. The point about all such proposals is that conditions of adequacy on any system of semantic categorization are imposed by considerations external to it. This means that instances of synonymity supply a criterion for the system, and not vice versa. If, for example, a semantic analysis is to be given of English terms for domestic animals, then $\underline{\operatorname{dog}}$ and cat will turn out to be synonymous if 'quadruped' and 'carnivorous' are the only two relevant semantic labels the system provides. To say that this result shows the system to be absurdly inadequate is doubtless correct; but that can only be said if it can

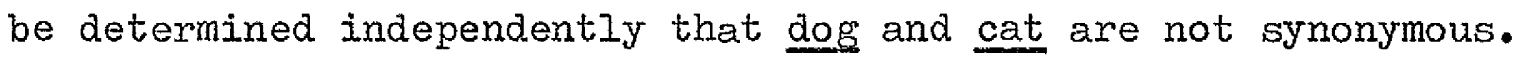

8. Abraham \& Kiefer 1966. 
$6 a$

1. Synonymy, form and meaning 
1.0 It is important to distinguish at the outset between two related but separate issues: (i) whether or not any language could have expressions differing in form but not in meaning, and (ii) whether or not any language does have expressions differing in form but not in meaning. The former is a matter of relations between definitions, i.e. the only sense in which no language could have expressions differing in form but not in meaning would be if we chose to define 'form' and 'meaning' in such a way that it followed from our definitions that any statement to the effect that expressions $\underline{a}$ and $\underline{b}$ differed in form but not in meaning would be selfcontradictory. If, on the other hand, we do not so define 'form' and 'meaning' the possibility is open that any language may have expressions differing in form but not in meaning. The question whether any language does have such expressions is a subordinate question, in that whether it arises at all depends on the answer propounded to the question of definitions.

1.I Let us consider the following postulate:

(PI) The expressions of a language differ one from another in form or meaning, but not necessarily in both.

That PI constitutes a reasonable and, for purposes of linguistic analysis, an indispensable minimal assumption may be concluded from the following argument.

If a linguistic expression is regarded as an item characterized in the dimensions of form and meaning, such that in order to recognize any expression it is necessary to know its form, and in order to understand any expression it is necessary to know its meaning, then 
unless we treat some expressions in a language as having different meanings from others, and some expressions as having different forms from others, it becomes impossible to explain how a language is successfully employed for purposes of communication. That is to say, either of the hypotheses (i) that all expressions of the language have the same form, or (ii) that all expressions of the language have the same meaning, fails to provide a satisfactory basis on which to account for the ways in which, by means of discourse, one languageuser understands and can be understood by others. For on neither of the hypotheses mentioned would difference in form be correlatable with difference in meaning. An analysis based on either hypothesis would thus be unable in principle to account for the successful use of a language in communication situations.

It follows from the assumption that some expressions differ in form and some in meaning that either some of those expressions differing in meaning will differ also in form, or some of those differing in form will differ also in meaning. Thus three possibilities must be envisaged. First, there will be cases in which two expressions differ both in form and meaning. Second, there will be cases in which a difference of meaning between two expressions is not accompanied by a difference of form: in such cases we may speak of 'homonymous expressions'. Third, there will be cases in which a difference of form between two expressions is not accompanied by a difference of meaning: in such cases we may speak of 'synonymous expressions'. 
The concept of synonymy thus takes its place in a framework of assumptions according to which any linguistic expression is taken to be determinate in respect of both form and meaning, but in which sameness in respect of form does not imply sameness in respect of meaning, nor vice versa.

1.2 Acceptance of the foregoing argument may appear to involve taking the position that 'synonymy' is a derived concept, defined in terms of the prior concepts of 'form' and 'meaning'l.

An objection to treating synonymy 'in terms of a prior notion of 'meaning' independently defined' is voiced by Lyons', who contends that if two expressions are synonymous this 'fact' must be regarded as 'part of' the meanings of the expressions in question.

This somewhat obscurely formulated doctrine seems to take it for granted that synonymous expressions do occur in (some) natural languages, and it must therefore assume postulates of linguistic analysis (although these are not explicitly stated) from which the possibility of synonymity may be inferred. Lyons is reluctant to accept, however, what he regards as the usual view of 'semanticists'. The typical approach of 'semanticists' is described by Lyons in the following terms:

1. A concept is derived if it is one defined in terms of prior concepts belonging to the science in question (e.g. 'velocity' in physics, defined in terms of the prior concepts of 'time' and 'distance') and primitive if it is not thus defined (as e.g. 'time' in physics).

2. Lyons $1963 \mathrm{Ch} .4$. Cf. also Lyons $1968 \mathrm{Chs} .9$ and 10. 
'the question whether two forms are synonymous (has a the same meaning as $\underline{b}$ ?) is reduced to the question whether the two forms designate the same entity, their 'meaning'. Thus, if it is established that a in one of its 'meanings' signifies $\underline{x}$ (the different theories being distinguished by the manner in which they describe $\underline{x}$ ) the test of synonymy of $\underline{a}$ with $\underline{b}$ is whether $\underline{b}$ also, in one of its 'meanings', signifies $x^{3}$.

This view Lyons contrasts with the one he is advocating, namely that: 'a is not synonymous with $\underline{b}$ because of its meaning; the fact of their synonymy is part of their meaning ${ }^{4}$.

3. Lyons 1963 \$4.3. Cf. Lyons 1968 \$9.4.2: The second assumption commonly made by semanticists is that synonymy is a relation of identity holding between two (or more) independently-defined senses. In other words, the question whether two words, $\underline{a}$ and $\underline{b}$, are synonymous is reduced to the question whether a and $\underline{b}$ denote. the same entity, their sense'. There appears, when one compares, Iyons 1963 with Lyons 1968, to be some reassessment of the 'semanticists' position (marked by restriction of the discussion to 'words', and the replacement of meaning by sense, and of designate by denote). But in both cases lyons is careful to attribute to the 'semanticists' a fallacy about the ontological status of meanings (cf. Alston 1964. pp. 19-22). The suggestion is, clearly, that their approach to synonymy must be wrong because their view of meanings is wrong. Put in to bat on this impossible wicket, the 'semanticists' are cheaply dismissed. But the tactic would not work against a team e.g. of Wittgensteinian 'semanticists'. For one can hold that the question whether $a$ and $b$ are synonymous can be reduced to ( $i$ ) what is the meaning of a?, $(i i)$ what is the meaning of $\underline{b}$ ? and ( $i i i$ ) are these two meanings identical? without simultaneously holding the view that meanings are entities. This is the real issue, which the manoeuvre against the 'semanticists' evades.

4. Lyons 1963 \$4.3. Cf. Lyons 1968 \$9.4.2: The synonymy of lexical items is part of their sense'. 
Two features of the contrast drawn by Lyons call for comment. First, there is a failure to distinguish between questions of definition and questions of establishing facts. The 'semanticist' who proceeds as described by Iyons is not by implication committed to the thesis that $\underline{a}$ is synonymous with $\underline{b}$ 'because of' its meaning (it would be nonsense to say that Jones is as tall as Smith because of his height) but to the thesis that a's being synonymous with $\underline{b}$ consists in their having the same meaning, although differing in form. That is to say, the 'semanticist' can legitimately regard himself as entitled to claim that $\underline{a}$ and $\underline{b}$ are synonymous when he has established that they have the same meaning. Whereas, according to Lyons, to establish the fact that $\underline{a}$ and $\underline{b}$ are synonymous is, presumably, mexely to establish 'part of' their meaning. But no more sense can be made of this contention than of the contention that to establish that Jones is as tall as Smith is to establish 'part of' their height. On the other hand, if all that Lyons is contending is that the fact that $\underline{a}$ and $\underline{b}$ are synonymous is merely one of many semantic facts about these expressions, doubtless the 'semanticist' would agree, and consequently the alleged contrast between his position and Lyons's collapses. Second, if we are not to set about determining synonymity by investigating whether $\underline{a}$ and $\underline{b}$ have the same meaning, i.e. if we are not allowed any appeal to a prior concept of meaning, it becomes incumbent to ask: what, then, are we asking when we ask whether a and $\underline{b}$ are synonymous? Lyons's answer to this, surprisingly, is that we are asking whether $\underline{a}$ and $\underline{b}$ are interchangeable without change 
of meaning, this being, allegedly, the criterion employed by 'unsophisticated speakers'.

'When questions of sameness of meaning arise for unsophisticated speakers, no appeal is made to an abstract entity of 'meaning': a given word or phrase is accepted as having the same meaning as another word or phrase if its substitution for the other in the given context yields an utterance which they will accept as having the same meaning as the first utterance. 5

This, claims lyons, is 'not so circular as it may sound'. It is, however, just as circular as it sounds, i.e. we cannot sensibly contend that we are not asking whether $\underline{a}$ and $\underline{b}$ have the same meaning if our explanation of what we are asking is merely that we are inquiring whether certain larger units, of which a and $\underline{b}$ are parts, have the same meaning. The question is merely deferred: it does not thereby change its character, and cease to be a question about 'meanings'. On the contrary, all that happens is that we have changed the units about whose 'meanings' we are inquiring. But the enterprise of defining 'synonymy' independently of a prior concept of 'meaning' has made no progress.

1.21 The main argument used by Lyons in support of his view of meaning relations is an argument from analogy, based on remarks by Reichenbach

5. Lyons $1963 \$ 4.46$. 
about the concept of 'weight'.

'What is the weight of a body? It is usually conceived as an abstract property of the body, recognizable from certain physical effects. Using Russell's principle of abstraction we can reduce the concept weight to the relation having the same weight. The weight of a body is the class of all objects having the same weight as this body. An adept in traditional logic would object that in order to define the same weight we must first define the weight, and then proceed by addition of the differentia specifica to the genus. But there is no reason to insist on this impractical method. It is admissible to conceive the notion of the same weight as prior to that of weight and to define the latter in terms of the former. This conception corresponds to the actual procedure used in the empirical ascertainment of the weight of a body. The balance is a device which indicates, not the weight, but equality of weight ${ }^{1}$.

\section{Lyons comments:}

'The difference between this view of the 'abstract qualities' and a more ancient conception, which regarded them as positive properties inherent in things, is

1. Reichenbach 1947 p. 210. 
readily appreciated. We have only to think, for example, of Plato's difficulty in connexion with the simultaneous predication of 'tallness' and 'shortness' of the same person. It seems to me that many of the difficulties experienced by semanticists in the treatment of meaning-relations such as synonymy or antonymy are of a similax nature, being caused by their view of 'meaning' as prior to these relations ${ }^{2}$.

But to argue thus is to leave out of account the most important feature of the case, namely the particular advantage of treating a concept like 'weight' as a comparative concept rather than as the concept of an inherent property. For the 'weight' of a body varies according to where it is weighed, the 'length' of a body is not invariant under all physical conditions, and so on. But to apply such considerations to the treatment of 'meaning' is to force a quite spurious analogy upon the case. For the option is not, as Iyons implies, a simple choice between treating 'meaning' as an inherent property of expressions and treating it as a comparative concept, as in the case of 'weight'. And since the idea that the 'meaning' of an expression is an inherent property of that expression (or is determined by inherent properties of the expression) can be taken no more seriously than the idea that being a kettle is an inherent property of a particular piece of metal, knocking down this Aunt Sally hardly

2. Iyons $1963 \$ 4.3$. 
vindicates Lyons's view of semantic relations 3 .

If, moreover, Lyons were genuinely basing his approach on Russell's 'principle of abstraction', he would have to argue that the meaning of an expression is a certain class of expressions 4 , whereas he wishes to argue for a different conclusion: that the 'fact' of expressions being synonymous is 'part of' their meaning.

But the insuperable objection to the argument from analogy is this: that the treatment Reichenbach describes is limited in its application to a particular type of empirical concept, namely measurement concepts in the physical sciences, i.e. concepts fundamental to which is the ultimate appeal to a standard 5 . There is simply no parallel here at all to the concept of 'meaning' in linguistics. In no sense do natural languages have 'standard meanings' arbitrarily set up, by reference to which other 'meanings' are measured, and since this is the plain fact of the matter, there is no sense in which 'sameness of meaning' takes priority over 'meaning' where natural languages are concerned.

That it is the absence of appeal to standards which vitiates Iyons's assimilation of the case of 'meaning' to the case of 'weight'

3. Iyons does not explain how the 'semanticist' is supposed to reconcile the view that meanings are inherent properties with the view that meanings are entities. Perhaps this is another of the 'semanticist's' confusions: or perhaps he is allowed to opt for one or other of two equally untenable positions.

4. i.e. 'the meaning of an expression is the class of all the expressions synonymous with it' (Quine 1943 p. 120).

5. Cf. Iyons 1968 10.1.1. 
is readily apparent if we compare accounts which might be given of 'knowing the weight of $\underline{x}$ ' and 'knowing the meaning of $\underline{x}$ '. Here it will be seen that to treat the $f$ of $x$ as the class of all objects having the same $\frac{\mu}{\tau}$ as $\underline{x}$ will not do at all. For example, we should certainly not regard someone as knowing the weight of $\underline{x}$ if all he knew was that it had the same weight as various other objects, and only those. Nor should we regard someone as knowing the meaning of $x$ if all he knew was that there were no other synonyms than $\underline{y}$ and $\underline{z}$. We should, however, regard someone as knowing the weight of $x$ if he knew that it weighed the same as any other single object of which he knew the standard weight value. This is the essential point for a correct evaluation of the comparison with 'synonymy' and 'meaning'. In other words, we should not regard someone as knowing the meaning of $\underline{x}$ if all he knew was that it was synonymous with $\underline{y}$, but did not know the meaning of $\underline{y}$ either.

Finally, it must be observed that even in the case of concepts of measurement in the physical sciences, to talk e.g. of 'sameness of weight' as taking priority over 'weight' is a mere façon de parler. What this facon de parler is intended to bring out is the nature of the concept 'weight' as employed in these sciences. But to assert that 'weight' is conceptually derived from 'sameness of weight' would be, stricto sensu, as untenable as to assert that 'aeroplane' is conceptually derived from a prior 'same aeroplane'. We cannot, under pain of self-contradiction, incorporate into the analysans of a prior concept any concept which we claim to be derived therefrom. 
If we accept the façon de parler, however, it may be noted that there is no difficulty with the concept of 'form' which parallels that arising over 'meaning': 'sameness of form' is, on the contrary, most satisfactorily regarded as prior to 'form'. When we ask whether a person knows whether the lecturer said "bust" or "must", i.e. when we require someone to make an identification of linguistic form, we are indeed asking him to do no more and no less than identify the class of items having the same form. For there would be no sense in the objection that he might only produce further examples of items having the same form, but not know what form they had either.

This difference between the concepts of 'form' and 'meaning', whereby 'form' is to be treated as derived from 'sameness of form' but 'meaning' as prior to 'sameness of meaning', would appear to be fundamentally characteristic of the conceptual framework upon which linguistic analysis rests.

1.3 If PI (1.1) is accepted, the question of excluding the possibility of synonymous expressions arises only if it is maintained that linguistic analysis requires some further postulate or postulates compatible with PI but precluding the possibility of difference in form without difference of meaning.

The view is advanced by Bloomfield ${ }^{l}$ that linguistic analysis is based upon a postulate which requires us to suppose that within a given natural language there are no synonymous expressions. Bloomfield 
does not make out a detailed case, but the postulate in question is stated to be:

(P2) In certain communities (speech-communities) some speech-utterances are alike as to form and meaning'.

Bloomfield maintains (a) that this postulate obliges us to suppose that phonemic difference is invariably concomitant with difference of meaning, e.g. 'that each one of a set of forms like quick, fast, swift, rapid, speedy differs from all the others in some constant and conventional feature of meaning', and (b) that the postulate implies that difference of meaning is compatible with phonemic identify, e.g. 'in English, the phonetic form [be a] occurs with three different meanings: bear 'to carry, to give birth to', bear 'ursus', and bare 'uncovered''. Thus, according to Bloomfield, homonymity is possible, but synonymity excluded.

However, this conclusion does not in fact follow from the single postulate as Bloomfield formulates it, since the assertion that some utterances are alike in form and meaning does not preclude the possibility of synonymous expressions. There would be no difficulty in formulating a postulate which did, e.g.

(P3) The expressions of a Ianguage differ one from another in form or meaning, and if in form then al.so in meaning.

But the question which must be considered is whether there is any good reason for adopting a postulate such as P3. 
1.31 The denial that synonymous expressions occur in natural languages might be based on the adoption of a definition of 'meaning' such that it would then be inconsistent to claim that two expressions had the 'same meaning', as e.g. if the 'meaning' associated with any given form were to be defined as 'the total network of relations entered into' 1 by that form. However, a definition of 'meaning' which has the effect of rendering difference of form a sufficient condition of difference of meaning makes it impossible to have synonymous expressions even in a constructed communication system or symbolic language. This consequence shows that any such definition is not a definition of 'meaning' in the sense with which we are here concerned; for if one thing is clear it is that in principle we can, arbitrarily, modify a constructed language by the introduction of synonymous expressions ${ }^{2}$, (just as a government can introduce new coins having the same value as old coins, and thus modify the existing currency system).

1. This 'Firthian' definition is offered by J. C. Catford (Catford 1965, \$5.I). If the 'meaning' of a is the total network of relations entered into by $a$, and the 'meaning' of $b$ is the total network of relations entered into by $b$, and $a$ and $\bar{b}$ are nonidentical, then the 'meaning' of the one cannot be the same as the 'meaning' of the other.

2. E.g. Dr. Zamenhof might have decided (as a whimsical afterthought) to have two Esperanto words for 'electricity' instead of one. The committee responsible for the International Code of Signals might decide to introduce a new signal as an optional alternative to one of the existing signals. (If it is denied that 'synonymous expressions' can thus be created by fiat (cf. Quine 1961 \$2), we must simply abandon the term 'synonymous expressions' as being tied to an inappropriate concept of 'meaning', and find some alternative way of talking about the relation in question). 
1.32 A more interesting denial of synonymity would be one based upon the thesis that a natural language, unlike a constructed language, is such that the conditions for synonymity of two expressions are never fulfilled. P3, it might be suggested, can be supported by an argument along the lines advanced by White ${ }^{1}$. White's analysis of synonymy may be summarized as follows.

Synonymy is held to be sameness of meaning of different expressions. If this is correct, then to interpret aright claims that a is synonymous with $\underline{b}$ we need to clarify (i) the 'sameness' involved, and (ii) the 'meaning' involved.

(i) At least four different kinds of 'sameness' may be distinguished. We use the word 'same' in connexion with various parts of the history of one continuous thing. For example, to whatever position in the room a chair is moved, we still regard it as the same chair. We will correct an acquaintance who mistakenly supposes that the chair that used to stand by the window has been sold and that there is a new chair that stands by the door. "No," we say, "it's the same chair". Similarly, we refer to "the same man we saw yesterday". Such cases are examples of 'type-l sameness'. Second, there is the kind of case in which we have two or more instances of non-continuous things ('type-2 sameness'), as when we talk about "the same dance step" or "the same experiment", alluding to a repetition of previous actions. Then there is the kind of case where we recognize sameness in two or more coexistent copies of one thing ('type-3 sameness'), as when we talk 
about "the same newspaper", "the same curtains", "the same motor car" or "the same gramophone record". In such cases there may or may not be a prototype over and above the copies. Finally, there is the kind of case where we say that at least two continuous things are the same in a given respect ('type-4 sameness'), as when we tell someone that he has the same eyes, or the same bearing, or the same manner as his father. We use this formula even when the comparison between $A$ and $B$ hinges on a detachable object which is not part of them, as when we say "they have the same room", meaning that they share it. There are, however, English idiomatic differences corresponding to this latter distinction. For if the respect in question is a non-detachable, non-interchangeable characteristic (like manner, limp, hair, or even as a marginal case - income) it is usual to say not only that $A$ and $B$ "have the same X", but also that they are "alike in" or "in respect of" $\mathrm{X}$; whereas if the respect involves a detachable, interchangeable object (Iike room, car or hat) it is usual to say that $A$ and $B$ "have the same X", but hardly that they are "alike in" or "In respect of" X. 'Type-4 sameness' may be further analysed as follows. If $A$ and $B$ are the same in respect of a given object, we may classify the sameness of that object under 'type-1 sameness' or 'type-3 sameness'. That is, it will either be one and the same object which $A$ and $B$ share (e.g. room or garden fence) or, on the other hand, there will be two distinct copies of the same sort (e.g. motor car). But the question of whether the object counts as one or two does not depend on how many possessors it has. However, if $A$ and $B$ are the same in respect of a given charac- 
teristic, it is not always easy to decide whether we are dealing with one characteristic or two, and the question of how many possessors it has becomes more important. There are two general arguments for classing such characteristics under 'type-3 sameness'. One is that it makes perfectly good sense to say in such cases that there is one $X$ (e.g. Iimp, interest, hair-do) which $\mathrm{A}$ has, and another $\mathrm{X}$ which $\mathrm{B}$ has, but no difference between them. This is supported by the second argument, that it is possible for someone to know that both $A$ and $B$ have $X$, but not to recognize it as the same characteristic.

The sameness involved when it is claimed that two expressions "have the same meaning" is 'type-4 sameness', i.e. the claim maintains that two given expressions $\underline{a}$ and $\underline{b}$ are the same in respect of $x$, their meaning. If we accept this, then the further question arises of assessing 'meaning' as a respect in which instances of 'sameness' may occur.

(ii) 'Meaning', in the sense in which it may be claimed that two expressions "have the same meaning", is most satisfactorily regarded as a characteristic in respect of which the expressions are the same. This characteristic is the way or ways in which the expressions are employed by language-users. Thus there are general arguments (see above) for classing the meaning which expressions share under 'type-3 sameness'.

But this conclusion is just the one which raises insuperable difficulties as far as determining synonymity is concerned. For 'type-3 sameness' for characteristics is the case where sameness is in the eye 
of the beholder, i.e. where there is no objectively verifiable 'same object' involved, and all depends on whether we choose to pay attention to the similarities and ignore the differences. It is typical of 'type-3 sameness' for characteristics that there is always some viewpoint from which two allegedly same respects can, in principle, be differentiated. Hence if we believe that there are instances where two expressions cannot be differentiated in respect of meaning, we must be deceiving ourselves. This is not to say that we shall not find instances where a plausible case can be made out for saying that two expressions are synonymous: but that the decision will be arbitrary, in the sense that there will always be - if we choose to look for it some reason for denying that the meaning is 'the same'.

Thus the case of allegedly synonymous expressions is like that of the spanner and the wrench. We may say that a spanner and a wrench have the same use, namely to tighten and loosen nuts, or that they have different uses, because the way a spannex tightens a nut is not exactly the same as the way a wrench does, and besides there are other uses which wrenches have and spanners do not. Similarly, e.g. the expressions brother and male sibling may be alleged to be synonymous, and there is a good reason for this, namely that both are used to specify a certain family relationship. On the other hand, reasons may be found for denying that they are used in exactly the same way, e.g. that one expression provides a translation of the French word frère, while the other does not, or that one expression explicitly analyses the relationship in question, whereas the other does not. We thus have 
a choice of asserting or denying that these two expressions are synonymous, depending on whether we wish to call attention to the similarities of meaning, or the differences.

In general, the following are ways by one or other of which we may always differentiate between the meanings of any two expressions if we so wish: (a) by showing that the meaning of a is known to a person $X$, when the meaning of $\underline{b}$ is not; ( $b$ ) by showing that the meaning of a may be rendered in some other language by an expression which is not an exact translation of $\underline{b}$; (c) by showing that a differs in the organization of meaningful elements from $\underline{b}$; (d) by showing that the meaning of a differs in some respect other than intension or extension (e.g. emotively) from the meaning of $\underline{b}$; (e) by showing that a has for a person $X$ associations not shared by $\underline{b}$.

Thus there will always be something true of the meaning of a which is not true of the meaning of $\underline{b}$, even in cases where some other consideration might induce us to say that the meanings of $\underline{a}$ and $\underline{b}$ were 'the same'.

1.33 White's position may be compared with that taken up by Nida, who holds that the non-occurrence of synonymous expressions in natural languages is a 'principle of semantic analysis'. In support of this contention, Nida observes that certain expressions 'ordinarily listed as synonyms' turn out, upon investigation, not to be identical in meaning. Bxamples are: peace and tranquillity, childish and puerile, truth and reality. This holds also, in Nida's view, for variant pronunciations of a given word: 
'For example, the alternant pronunciations of duty (1)/duwtiy/ and (2)/dyuwtiy/ carxy certain distinct connotations. In some circumstances the form /dyuwtiy/ induces an unfavorable response from the listener, who interprets it as pedantic or associated with people whose culture he does not appreciate. On the other hand, among a certain small set of speakers of American English the form/duwtiy/ is a mark of educational and cultural inferiority. The alternant pronunciations of creek / kriyk/ and / krik/ bear similar distinctions, but to different types of speakers. If alternant pronunciations of morphemes do nothing more than identify the dialect area, they are to that extent nonequivalent, 1 .

Thus according to both White and Nida close investigation of the use of expressions in a natural language will always reveal some reason for denying their synonymity.

But pointing out various ways of distinguishing between alleged synonyms is the traditional pastime of synonymists. Collinson lists nine possible differentiae:

(1) One term is more general and inclusive in its applicability, another is more specific and exclusive, e.g. refuse / reject. Cf. seaman / sailor, ending /inflexion, go on foot / march.

(2) One term is more intense than another, e.g. repudiate 


\section{/ refuse. Cf. immense / great, towering / tall.}

(3) One term is more highly charged with emotion than another, e.g. repudiate or reject / decline. Cf. looming / emerging, louring / threatening.

(4) One term may imply moral approbation or censure where another is neutral, e.g. thrifty / economical, eavesdrop / listen.

(5) One term is more "professional" than another; e.g. calcium chloride / chloride of lime / bleaching powder; decease / death; domicile / house; to ordain a priest, institute or induct a vicar, consecrate or instal a bishop / appoint a professor.

(6) One term belongs more to the written language, it is more literary than another, e.g. passing / death. The literary language includes further distinctions like the poetical and the archaic.

(7) One term is more colloquial than another, e.g. turn down / refuse. The spoken language, too, includes further distinctions like the familiar, slangy and vulgar.

(8) One term is more local or dialectal than another, e.8. Scots flesher / butcher, or to feu / to let.

(9) One term belongs to child-talk, is used by children or in talking to children, e.g. daddy, dad, papa / father (in which different social levels are 


$$
\text { discernible), teeny / tiny, etc. }{ }^{2}
$$

Ingenuity might find ways of adding considerably to this list. But however long the list of differentiae, it will still fall short of providing the required support for P3, unless reason is shown for supposing that any pair of expressions must be differentiable on at least one such count. 3 For it would be a misapplication of Leibniz's law to proceed on the assumption that establishing that something is true of the meaning of a which is not true of the meaning of $\underline{b}$ is sufficient to show that $\underline{a}$ and $\underline{b}$ differ in meaning ${ }^{4}$. To clinch White's

2. Collinson $1939 \mathrm{pp}$. 61-62. The analysis is based on that given by Devoto in the article "Sinonomia" in the Enciclopedia Italiana Vol. XXXI, p. 857.

3. This applies whether or not we accept the validity of particular differentiae. T.g. one might question the assumption, apparently accepted by Collinson, Nida and White, that to establish that different speakers have different attitudes to the use of $a$ and $\underline{b}$ is sufficient to establish that $a$ and $b$ differ in meaning. (This leads Ziff (Ziff 1960, $\$ 179$ n.3) to dismiss Nida's conclusion that natural languages do not have synonyms as based on a confusion between 'meaning' and 'connotation'.)

4. Analogously, one could never conclude that two articles in a shop cost the same, since certain things might be true of the price of one article which were not true of the price of the other, e.g. that one price had been determined by the shop manager and the other by the wholesaler, or that one price was regarded by the customer as cheap, and the other as dear, etc. But to anyone who argued thus, it would be correct to reply: these are certainly possible ways of differentiating between the two prices, but they have nothing to do with establishing whether or not the prices are the same. 
argument, it would need to be shown that the various criteria for synonymity are interrelated in such a way that any pair of naturallanguage expressions satisfying one or some of these criteria necessarily fail to satisfy another or others. This final step in the argument is the step missing.

1.34 A prima facie case for rejecting P3 can be made out if it can be show that natural languages have pairs of sentences whose difference in meaning depends on a condition that could be falsified. This seems plausible in the case of many pairs of sentences containing extensional expressions ${ }^{1}$. For example, the difference in meaning between

\section{(1) John wore shoes}

and (2) John wore boots seems to depend entirely on the difference in extension of the words shoe and boot; for we cannot detect any differences in e.g. grammatical structure, idiomatic usage, register, 'emotive overtones' etc. which contribute in any way to differentiating the meaning of (1) from the meaning of (2). Thus we can say that if - contrary to the facts of the case - shoe and boot were co-extensional terms, then (1) and (2) would not differ in meaning. But to say this is to concede that there could be a natural language exactly like English except in that shoe and boot would be, in this hypothetical language, co-extensional terms. In such a language (1) and (2) would be synonymous.

1. Extensional expressions are such expressions as chair, which is said to have as its 'extension' the class of chairs, so that when we apply this predicate to some object (by saying of it that is "is a chair") we are asserting that the object in question is a member of the class of chairs. 
Granted the reasonableness of the premisses, we should nonetheless be prevented from reaching this conclusion if some general reason could be adduced for supposing that no two extensional expressions can have the same meaning, even if they do not differ in idiomatic usage, register, 'emotive overtones' etc.; since if shoe and boot, although co-extensional, differed in meaning this would provide grounds for denying the synonymity of (1) and (2).

The only serious argument in support of the claim that extensional expressions which differ in form must also differ in meaning (even when identical in respect of idiomatic usage, register, 'emotive overtones' etc.) is one advanced by Goodman ${ }^{2}$. Goodman points out that identity of extension would be an adequate criterion of synonymity for most pairs of extensional expressions, at least if we were restricting our interest to such aspects of meaning as affect the truth or falsehood of assertions. Even so, a problem arises if we wish to include within the scope of our definition extensional expressions of which the extension is the null class. For according to the criterion proposed, such expressions as unicorn and mountain higher than Everest would be synonymous, since they coincide in extension. But if it is the case that Mary thought she saw a unicorn in the garden, she would be telling the truth if she said "I thought I saw a unicorn in the garden", but uttering a falsehood if she said "I thought I saw a mountain higher than Everest in the garden".

2. Goodman 1949. 
Goodman's resolution of this difficulty is to propose the following amendment of the suggested account of synonymy for extensional expressions. An expression such as chair may be regarded not only as having an extension in the usual sense, but also as having 'secondary extensions'. A secondary extension will be defined as the extension of a compound expression in which the expression chair appears as a constituent part, e.g. the expression picture of a chair. The amended criterion of synonymity proposed is: 'identity of primary and secondary extensions'. That is, $\underline{a}$ and $\underline{b}$ are synonymous if and only if they coincide not simply in respect of their extension in the usual sense, but also in respect of the extensions of all matching compounds which include $\underline{a}$ and $\underline{b}$ as parts. The amended criterion now seems adequate to cope with cases like unicorn and mountain higher than Everest, since there will be matching compounds (e.g. picture of a unicorn and picture of a mountain higher than Iverest) which do not agree extensionally and thus rule them out as a synonymous pair.

However, acceptance of the amendment has the consequence that no two extensional expressions $\underline{\mathrm{a}}$ and $\underline{\mathrm{b}}$ can be regarded as synonymous. For the expression chair that is not a stool can be correctly applied to any chair but to no stool: it qualifies, so the argument runs, as a chair-description but not a stool-description. Thus the expressions chair and stool differ in secondary extension, for there are matching compounds, namely chair-description and stool-description, which differ in extension. Now in the expression chair that is not a stool we might substitute for chair and stool any two nonequiform expressions $\underline{\mathrm{a}}$ and $\underline{\mathrm{b}}$, 
and arrive at the same result. Whereas if we insert the same expression a in both places, we have an expression of the type chair that is not a chair - and such an expression, whatever its status, clearly cannot simultaneously be and not be a chair-description. The conclusion is that no two extensional expressions can be synonymous.

Goodman's case must, however, be rejected. For what is not explained is why anyone should accept failure to differ in secondary extension as a sufficient condition for difference of meaning between two expressions which coincide in primary extension and differ in secondary extension only in virtue of the fact that they differ in form. For example, granted that the words furze and gorse have the same primary extension, it seems that the case for saying that furze that is not gorse counts as a 'furze-description' but not as a 'gorsedescription' must rest ultimately upon formal features of that expression, i.e. 'furze-description' must here be explicated in some such fashion as 'description using the word furze ...'. For if any other word than furze were used, then by Goodman's own argument we should not have a 'furze-description' that was not a 'gorse-description'. But if that is so, then all the difference in secondary extension assures us of is that furze and gorse differ in form, which was known at the outset. If we take difference of secondary extension in such cases to give a guarantee of difference in meaning, we reach the paradoxical position that under no circumstances - e.g. in a fairy tale, or a science fiction story - could an author invent a race of imaginary creatures and give them two alternative names (e.g. trugs or 
bogglewits) without ipso facto creating two words with different meanings. But this conclusion conflicts, clearly, with the principle that synonymous expressions may be created by fiat (cf. 1.31); for neither English nor any invented language with the grammatical apparatus for constructing 'trug-descriptions' and 'bogglewit-descriptions' could have trug and bogglewit as synonyms. Thus, even as regards the meanings of expressions having the null class as their extension, Goodman's proposal is defective. For while it supplies an 'extensional' explanation of the difference between, say, unicorn and cockatrice, it does so at the expense of not being able to account for the lack of difference between cockatrice and basilisk. A picture of a unicorn is not a picture of a cockatrice: but a picture of a cockatrice is a picture of a basilisk.

If the argument from secondary extensions is rejected, it seems that we can say ( $i$ ) that there are expressions (belonging to the class of extensional expressions) which differ in meaning only if they differ in (primary) extension, and ( $i i)$ that there may be some such expressions which do not in fact differ in (primary) extension, and thus do not differ in meaning. 3

3. Other arguments which would support P3 are difficult to find. Lyons observes that 'although it is frequently asserted ... that there are no 'real.' synonyms in natural languages, I am aware of no argument that gives a Iinguistically useful interpretation to the term 'synonymy' to support this assertion' (Lyons 1963 \$4.46). Ullmann says that instances of 'total synonymy' are 'a luxury which language can ill afford' (UlImann 1959 p. 108). But this is scarcely an argument; moreover, it is not clear what 'price' a language would have to 'pay' in order to 'afford' synonyms. Pike asserts that 'it is ultimately from an etic point of view, 
1.4 The case presented thus far in favour of accepting P1 but rejecting $P 3$ has assumed that a satisfactory account of some kind could be given of synonymous expressions in a constructed as opposed to a natural language. For the objection to positions which render difference in form a sufficient condition of difference in meaning was based on the consequence that synonymy would then be impossible even in the case of constructed languages. To make good this objection, the notion of synonymous expressions in a constructed language requires some clarification, and a fuller supporting account of 'form' and 'meaning' must be provided.

We may begin by pointing out that all that has so far been taken for granted about 'form' and 'meaning' is that it is in virtue of knowing the form of an expression that language-users are able to recognize instances of that expression, and in virtue of knowing the meaning of an expression that they are able to understand it when used in discourse. These assumptions fit equally well the case of a natural language or of a constructed sign system of any kind.

As regards natural languages, a distinction between formal and

alone, that one may claim that there are never any genuine synonyms' (Pike 1967 p. 613). Again, it is not clear what such a claim amounts to; nor are any supporting arguments given. Dixon says that 'there is no absolute synonymy' and links this with the assertion that 'there are patently no two lexical items which can occur in exactly the same linguistic environments' (Dixon $1963 \mathrm{pp} .43-44$ ). Why this must be the case is not explained. According to Southworth, 'it is probably true, as many linguists assume, that no two lexical items in any language are exact synonyms' (Southworth 1967 p. 346). But no grounds for regarding this as 'probable' are stated. 
semantic knowledge answers to certain elementary criteria one would apply in assessing language learning. For example, a Frenchman learning English might be taught the correct pronunciation or spelling of the word eight; but one would not wish to say that he knew the meaning of the word simply on the basis of his success in recognizing tokens of the type, or producing such tokens himself when called upon to do so. Or, on the other hand, he might be taught that there was an English word for the cardinal number ' 8 ', and he might even be taught, by means of partial translations with blanks, where that word ought to occur in English sentences as an equivalent of French huit. He might perhaps then be described as knowing the meaning of a certain Inglish word; but one would not wish to say he knew the form of the word in question until he knew e.g. how to fill in the blanks translating French huit. The distinction also matches different ways in which one would describe features of success or failure in language-using. For example, if I have temporarily forgotten the name of the small Hampshire village where my Aunt May lives, and so cannot comply with your request for her address, it is a form, not a meaning, which I cannot recall. But it is a meaning not a form which I have (partially) forgotten if I used to know the definition of typhlitis, but cannot now remember whether the term applies to cases of appendicitis or not.

Thus a distinction between formal and semantic knowledge may be supported in various ways. These may be paralleled in the case of constructed sign systems even of a very elementary kind; for example, the 'builder's Ianguage' described by Wittgenstein in $\$ 2$ of the 
Philosophische Untersuchungen ${ }^{1}$. One might in such a case describe the relevant linguistic knowledge by specifying (i) what patterns of articulated sound count as instances of utterance of the expressions Würfel, Säule, Platte and Balken, and (ii) what requirement the utterance of each of these expressions indicates. For this is the knowledge which would enable anyone to assume the role either of the builder or of his assistant in the communication situation envisaged. All that falls under (i) comprises formal knowledge, and all that falls under (ii) semantic knowledge. Someone might have the requisite formal knowledge without the requisite semantic knowledge, e.g. he might be able to recognize utterances of Würfel, Säule, Platte and Balken, but not know, or be mistaken about, which kind of item was to be brought in response to which kind of utterance. This is to be distinguished from not knowing the difference between utterances of Würfel, Säule, etc. From the point of view of an outside observer trying to construct a hypothesis about the Iinguistic knowledge shared by the builder and hi.s assistant, it would suffice to 'set up' four linguistic expressions, each differing from the others both in 'form' (i.e. in respect of what

1. 'Die Sprache soll der Verständigung eines Bauenden A mit einem Gehilfen B dienen. A führt einen Bau auf aus Bausteinen; es sind Würfel, Säulen, Platten und Balken vorhanden. B hat ihm die Bausteine zuzureichen, und zwar nach der Reihe, wie A sie braucht. Zu dem Zweck bedienen sie sich einer Sprache, bestehend aus den Wörtern: "Würfel", "Säule", "Platte", "Balken". A ruft sie aus; - B bringt den Stein, den er gelernt hat, auf diesen Ruf zu bringen. - Fasse dies als vollständige primitive Sprache auf.' 
sound sequences count as utterances of the expression) and in 'meaning' (i.e. in respect of what requirement the utterance of the expression indicates).

This situation is in all essentials that of the linguist engaged in the description of a natural language. From his point of view, Iinguistic expressions may be defined as theoretical constructs set up as units in terms of which to state linguistic knowledge, this knowledge being postulated to account for interpersonal communication by speech or writing.

The mechanism of explanation based upon such constructs is typically as follows. If on a given occasion $A$ communicates successfully with $B$ by uttering a certain sequence of sounds, we explain this by supposing that $B^{\prime}$ s linguistic knowledge enables him to recognize the sounds as a token of a certain type, and, in vixtue of belonging to that type, as being understandable in a certain way. We also suppose that $A^{\prime}$ 's linguistic knowledge enables him to select the appropriate sequence of sounds to utter, on the assumption that $B$ would recognize them as a token of the type in question, and understand accordingly. Mutatis mutandis, similar suppositions apply in the case where A communicates with $B$ by means of marks on a surface, or other visual as opposed to auditory signals. Communication is explained, in short, by supposing that acts of communication (speech acts, acts of writing) involve the instantiation of linguistic expressions by $A$, and $B^{\prime} s$ application of his own linguistic knowledge to their recognition and interpretation. For each linguistic expression set up, therefore, a specification 
is required of both 'form' and 'meaning'. A language I may be described by specifying a set of linguistic expressions, each characterized in respect of form and meaning.

Clearly, it does not advance the explanation of communicationin-I to multiply 'linguistic expressions' unnecessarily. If different linguistic expressions of $\underline{I}$ (the language under description) are to be set up to accommodate various formal and semantic distinctions, when we speak of two linguistic expressions, it is implied that there is a difference in form or in meaning, ox both. If there is no difference either in form or in meaning, there is no sense in which two linguistic expressions are involved, as distinct from two instantiations of the same expression. This proviso corresponds to the way in which rules are to be envisaged as governing a constructed communication system. E.g. it would be nonsense for Wittgenstein's builder and his assistant to agree (a) that Balken was the word to be uttered by the builder when he wanted a beam brought, and furthermore (b) that the requirement of a beam by the builder was to be indicated by his uttering the word Balken. To agree to both (a) and (b) would be merely to reiterate agreement to one and the same rule (i.e. the rule for the expression Balken), not to set up rules for the two expressions Balken and BaIken. To create synonymous expressions in a constructed language would be to agree that one and the same semantic rule should apply to two or more expressions recognized as distinct. E.g. the builder and his assistant, dissatisfied with Wittgenstein's meagre and unimaginative provision for their intercourse, might agree that (a) the requirement 
of a beam by the builder might be indicated by his uttering the word Balken, and that furthermore (b) alternatively, the very same requirement might also be indicated by his uttering the word Austerlitz. And they might proceed to agree upon using the names of other famous battles as optional alternatives to the other words, thus providing each of the expressions Würfel, Säule, Platte and Balken with a synonym. (They would thus be instituting rules in a manner no different from the mathematician who stipulates (i) 'Let the value of $\underline{x}$ be $3^{\prime}$ and (ii) 'Let the value of $\underline{y}$ be 31 ; nor from the logician who sets up an interpreted logistic system in which ' $\underline{E}$ ' and ' $\underline{G}$ ' are constants standing for the same two-place predicate, so that 'Fab' and 'Gab' are both interpreted as 'a is the father of $\underline{b}$ '.) Correspondingly, the linguist constructing a hypothesis about the builder's language, based on observation of its use, would be led to set up Balken and Austerlitz as synonymous expressions in order to account for the fact that the communicational purpose served by both words appeared to be the same.

From this point of view, the concept of synonymy may be considered part of the structure of explanatory hypotheses about communicationin-I.

The less complex the internal organization of $\underline{I}$, and the more limited the range of communication situations in which $I$ is used, the clearer will be the application of a distinction between expressions which differ in meaning and those which do not. We may expect matters to be complicated in the case of natural languages by (i) the formal complexity of natural languages, (ii) the semantic complexity of natural 
languages, and ( $\mathrm{iii}$ ) the fact that the scope and purpose of communicational exchanges may be far less clearly demarcated than in the use of a restricted sign system of a very simple kind. 
2. Synonymy and Phonological Analysis 
2.0 Questions of synonymy arise in connexion with phonology insofar as the assignment of a particular phonological form to an expression is not entirely independent of the assignment of a meaning to that expression. The interdependence of assignments of form and meaning characterizes what may be termed 'semantically based phonology'.

2.01 By 'semantically based phonology' is here meant the view that the basis for phonological classification of the sounds of a language is the extent to which meanings of words, phrases or sentences in the language remain invariant under sound substitution, phonologically distinctive and non-distinctive sounds being distinguished in terms of the possibilities of interchange without change of meaning. It is characteristic of semantically based phonology to define phonological units, such as the phoneme, in such a way as to involve an appeal to meanings, whereas it is characteristic of non-semantically based phonology to deny that appeal to meanings enters into such definitions ${ }^{1}$. It is assumed in semantically based phonology that at least some information about how speakers use words for communication is essential for phonological analysis, whereas it is claimed by the advocates of non-semantically based phonology that, at least in principle, correct phonological analysis is possible without

1. '... the phoneme is essentially a phonetic conception. The fact that certain sounds are used in a language for distinguishing the meanings of words doesn't enter into the definition of a phoneme' D. Jones Le Maŝtre Phonétique 3.7.44 (1929): quoted in Bloch 1948 p. 5 . 
any semantic information ${ }^{2}$. For non-semantically based phonology, therefore, questions of synonymy do not arise.

2.02 It falls outside the scope of the present study to adjudicate in general between the claims of a semantically based and a non-semantically based phonology. However, arguments invoking synonymy have sometimes been advanced as reasons for rejecting a semantically based phonology, and these merit examination in the present context. 2.I According to Chomsky', 'the central objection to meaning as a criterion of analysis has always been the obscurity of semantic notions' and synonymy is 'the most dubious part' of semantic theory. We must therefore realize what we are conceding if we claim 'that in order to construct a phonemic system it is necessary to know which utterances are different in meaning. To know difference in meaning is to know synonymy, and this is the central term of the theory of meaning. If accepted, then, this claim is an open admission that linguistic analysis must be based on precisely the most dubious part of semantic theory'.

2. 'It would be possible to group the sounds of a language into phonemes without knowing the meaning of any words' D. Jones loc. cit. 'It is certainly possible to establi.sh phonemic systems without having recourse to meaning at all' Ebeling $1960 \mathrm{p} .83$. Bloch 1948 admits the utility of semantic knowledge for the phonologist 'as a shortcut in the investigation of phonemic structure' but insists on the possibility in principle of dispensing with it - with, however, one important proviso: 'Theoretically it would be possible to arrive at the phonemic system of a dialect entirely on the basis of phonetics and distribution, without any appeal to meaning - provided that in the utterances of the dialect not all the possible combinations of phonemes actually occurred' (BIoch 1948 p.5 n.8).

1. Chomsky 1955, pp. 141-142. 
This general objection is backed up by more specific theoretical and methodological considerations. Semantically based phonology is represented by Chomsky as entailing acceptance of a particular 'synonymity criterion' 2 , namely

SCl two utterances are phonemically distinct if and only if they differ in meaning 3

or, in an alternative and somewhat more exact formulation,

SC2 'given two utterance tokens $U 1$ and U2, U1 is phonemically distinct from U2 if and only if U1 differs in meaning from $\mathrm{U}_{2}{ }^{4}$.

The interpretation of the 'synonymity criterion' as applying to utterance tokens is stressed; SCl 'cannot be accepted, as it stands, as a definition of phonemic distinctness. If we are not to beg the question, the utterances in question must be tokens, not types'. 5 However, explicated thus the 'synonymity criterion' is open to the fundamental objection that 'there are utterance tokens that are phonemically distinct and identical in meaning (synonyms) and there are utterance tokens that are phonemically identical and different in meaning (homonyms). 6 The proposed criterion is therefore 'false in both

2. Chomsky 1957, p. 95 n.3.

3. Chomsky 1.957, p. 94.

4. Chomsky 1955, p. 143.

5. Chomsky 1957, p. 95.

6. Chomsky 1957, p. 95. 
directions'7. Specific counterexamples cited are the following. 'Let $U 1$ be the utterance I saw him by the bank, meaning the bank of the river, and let $\mathrm{U} 2$ be the utterance I saw him by the bank, i.e. the First National Bank. Clearly the two utterances are different in meaning. Nevertheless they are phonemically identical. Thus it is not the case that if $\mathrm{UL}$ and $\mathrm{U} 2$ differ in meaning, then they must be phonemically distinct. Notice that we cannot appeal here to the fact that these physically distinct utterances are two occurrences of the same sentence, two tokens of the same type, because the problem at issue is precisely to determine which utterances (i.e. which distinct pieces of tape) are repetitions of one another or tokens of the same type. To make this appeal is thus to beg the question at issue completely'. 8 Synonyms provide counterexamples falsifying the 'synonymity criterion' in the other respect mentioned. 'Let UI and U2 be any two expressions with the same meaning, e.g: "he is a bachelor" and "he is an unmarried man." Or, if one is inclined to deny the existence of absolute synonyms, consider such pairs as /ekənamiks/ and /iykanamiks/, "ádult" and "adúlt", "advértisement" and "advertísement", / ræšən/ and /reyšan/, /rædiyeytar/ and /reydiyeytor/, etc., which often coexist in one person's speech and are clearly synonyms. Such pairs have the same meanings but are phonemically distinct. Hence it is not the case that if two utterances are phonemically distinct, then they must differ in meaning' 9 . It is thus clear, in Chomsky's view, that if we adopt

7. Chomsky 1955, p. 143; Chomsky 1957, p. 95.

8. Chomsky 1955, p. 143. Another homonymous counterexample cited in Chomsky 1957, p. 95 is that of "'metal" and "medal" (in many dialects)'.

9. Chomsky 1955, pp. 143-144. 
the synonymity criterion 'we simply get the wrong classification in a large number of cases ${ }^{10}$. He rejects in advance the defence that such cases are simply exceptions. 'We cannot circumvent this argument by holding that this rule ... holds for all cases except the rather special case of homonyms and synonyms. For one thing, these are by no means peripheral cases. For another, 'homonymity' and 'synonymity' are simply the names we give to exceptions to this rule, and any rule works except for its exceptions'. 11

2.11 Having argued against a semantically based definition of phonemic distinctness, Chomsky also attacks on similar grounds the thesis that the phonologist in practice requires semantic information in order to determine phonemic contrasts. 'Lounsbury argues in kis "A semantic analysis of the Pawnee kinship usage", Language 32.158-94 (1956), p. 190, that appeal to synonymity is necessary to distinguish between free variation and contrast: "If a linguist who knows no English records from my lips the word cat first with a final aspirated stop and later with a final preglottalized unreleased stop, the phonetic data will not tell him whether these forms contrast or not. It is only when he asks me, his informant, whether the meaning of the first form is different from that of the second, and I say it is not, that he will be able to proceed with his analysis" ${ }^{\sharp}$ As a general method, this approach is untenable. Suppose that the linguist records/ekinamiks/ and /iykinamiks/, /viksin/

10. Chomsky 1957, p. 95.

11. Chomsky 1955, p. 144 . 
and /fiymeyl in meaning. He will learn that they are not, and will incorrectly assign them the same phonemic analysis, if he takes this position Iiterally. On the other hand, there are many speakers who do not distinguish "metal" from "medal", though if asked, they may be quite sure that they do. The responses of such informants to Lounsbury's direct question about meaning would no doubt simply becloud the issue.

'We can make Lounsbury's position more acceptable by replacing the question "do they have the same meaning?" with "are they the same word?" This will avoid the pitfalls of the essentially irrelevant semantic question, but it is hardly acceptable in this form, since it amounts to asking the informant to do the linguist's work; it replaces an operational test of behavior (such as the pair test) by an informant's judgment about his behavior. The operational tests for linguistic notions may require the informant to respond, but not to express his opinion about his behavior, his judgment about synonymy, about phonemic distinctness, etc. The informant's opinions may be based on all sorts of irrelevant factors. This is an important distinction that must be carefully observed if the operational basis for grammar is not to be trivialized. ${ }^{1}$. 2.12 As a non-semantic method of determining phonemic contrast, Chomsky advocates the 'pair test' in the following form. 'Suppose that a linguist is interested in determining whether or not "metal" and "medal" are phonemically distinct in some dialect of English. He will not investigate the meanings of these words, since this information is clearly irrelevant to his purpose. He knows that the meanings are different (or he is simply not concerned with the question) and he is interested in determining 
whether or not the words are phonemically distinct. A careful field worker would probably use the pair test, either with two informants or with an informant and a tape recorder. For example, he might make a random sequence of copies of the utterance tokens that interest him, and then determine whether or not the speaker can consistently identify ther. If there is consistent identification, the linguist may apply an even stricter test, asking the speaker to repeat each word several times, and running the pair test over again on the repetitions. If consistent distinguishability is maintained under repetition, he will say that the words "metal" and "medal" are phonemically distinct. The pair test with its variants and elaborations provides us with a clear operational criterion in completely non-semantic terms ${ }^{1}$. This holds true, in Chomsky's view, even if the informant is asked to distinguish the utterance tokens in terms of meaning. 'One should not be confused by the fact that the subject in the pair test may be asked to identify the utterance tokens by meaning. He might just as well be asked to identify them by arbitrarily chosen numbers, by signs of the zodiac, etc. We can no more use some particular formulation of the pair test as an argument for dependence of grammatical theory on meaning than as an argument that linguistics is based on arithmetic or astrology'?

2.13 Chomsky examines, and also rejects, a somewhat different version of the 'synonymity criterion'. 'A weaker claim ... might be advanced as

1. Chomsky 1957, pp. 96-97.

2. Chomsky 1957, p. 99 n.1. 
follows. Suppose that we have an absolute phonetic system given in advance of any language, and guaranteed to be detailed enough so that every two phonemically distinct utterances in any language will be differently transcribed. It may now be the case that certain different tokens will be identically transcribed in this phonetic transcription. Suppose that we define the "ambiguous meaning" of an utterance token as the set of meanings of all tokens transcribed identically with this utterance token'. It might now be proposed that two utterances are phonemically distinct if and only if they differ in "ambiguous meaning." Chomsky concedes that such a proposal 'might provide an approach to the homonymity problem, if we had an immense coxpus in which we could be fairly sure that each of the phonetically distinct forms of a given word occurred with each of the meanings that this word might have. It may be possible to elaborate this approach even further to cope with the problem of synonyms. In such a way one might hope to determine phonemic distinctness by laborious investigation of the meanings of phonetically transcribed items in a vast corpus. The difficulty of determining in any precise and realistic manner how many meanings several items may have in common, however, as well as the vastness of the undertaking, make the prospect for any such approach appear rather dubious' ${ }^{I}$. 2.14 Apart from the practical difficulties involved, Chomsky advances a further reason for rejecting the attempt to elaborate a semantically based phonology. 'There is one further difficulty of principle that should be mentioned in the discussion of any semantic approach to phonemic distinctness. We have not asked whether the meanings assigned 
to distinct (but phonemically identical) tokens are identical, or merely very similar. If the latter, then all of the difficulties of determining phonemic distinctness are paralleled (and magnified, because of the inherent obscurity of the subject matter) in determining sameness of meaning. We will have to determine wen two distinct meanings are sufficiently similar to be considered 'the same'. If, on the other hand, we try to maintain the position that the meaning of a word is a fixed and unchanging component of each occurrence, then a charge of circularity seems warranted. It seems that the only way to uphold such a position would be to conceive of the meaning of a token as "the way in which tokens of this type are (or can be) used," the class of situations in which they can be used, the type of response that they normally evoke, or something of this sort. But it is difficult to make any sense at all out of such a conception of meaning without a prior notion of utterance type. It would appear, then, that even apart from our earlier objections, any approach to phonemic distinctness in semantic terms is either circular or is based on a distinction that is considerably more difficult to establish than the distinction it is supposed to clarify" 2.2 Chomsky's thesis may be considered as falling into two parts, one concerned with the definition of phonological units, the other with discovery procedures for the determination of such units in particular languages. Both parts involve arguments which invoke the concept of synonymy, and the arguments in question are in both cases open to objections, which must now be examined. 
2.21 A general objection which may be raised against Chomsky's thesis concerns the alleged difficulty of semantic investigations, and, in particular, of establishing cases of synonymity. It is doubtless true that if one does not have a clear concept of synonymy, one will find it difficult to establish criteria for instances of synonymity: but this trivial observation applies equally to the concept of phonemic distinctness and to any other concept. Quite a different question is the question whether an investigator who does have a clear concept of synonymy, or of meaning, or of any other semantic concept, will find it difficult to establish criteria because the concepts in question are semantic concepts. Chomsky's arguments appear to assume an affirmative answer to this question, but the case for this answer is never satisfactorily made out. The use of phrases such as 'inherent obscurity' seems to suggest the existence of some fundamental difficulty attaching to semantic concepts, but the nature of the difficulty is never made clear. In practice, there would appear to be no greater order of difficulty involved in establishing whether $\underline{a}$ and $\underline{b}$ differ in meaning than in establishing whether $\underline{a}$ and $\underline{b}$ are phonemically distinct, granted the possibility of carrying out practical tests with informants. If an investigator has a clear concept of the semantic information he wishes to elicit, there seems no general reason why, either in principle or in practice, he may not devise testing techniques to elicit it ${ }^{1}$. In the particular case of testing for sameness of meaning of utterance tokens, Chomsky raises an objection which reveals a misconception of

1. Such techniques need not be open to the kind of objection Chomsky raises against Lounsbury's direct question about meaning (2.II). 
the question at issue; for, he alleges, such tests are complicated by the possibility of recognizing various 'degrees' of similarity of meaning. The assumption is, clearly, that unless we have a prior theory of synonymy which settles this and similar issues, then we do not know what we are testing for. But the 'difficulty' Chomsky raises is an irrelevance. No semantically based phonologist need waste time wondering whether, because ['Iaijan] and ['taigo] in a particular context appear to relate to very similar animals, he ought to count the meanings as 'the same'; nor, mutatis mutandis, for any other 'semantic similarity'. In phonology, the theoretical question 'how similar' meanings must be to be 'the same' simply does not arise: all the phonologist need be concerned with is establishing whether or not, in that context, his informants make a distinction. If they do, then ['laijon] and ['taige] count as 'different': if not, they count as 'the same'. (On theoretical aspects of testing for differences of usage in semantically based phonology, see further below 2.42).

2.22 The strategy of Chomsky's argument against the hypothetical defender of the 'synonymity criterion' contains a number of other unsatisfactory features, of which the most relevant for present purposes concern the implausibility of Chomsky's concept of synonymy. It appears that Chomsky wants to include under the term 'synonymy' any type of instance where one utterance-token is - in some sense - assigned the same meaning as some other utterance-token, as well as meaning-equivalence of corresponding types. What exactly is involved in assigning meanings is left unclarified, but Chomsky's examples include very disparate types of 
case. If mere instances where there is a variation of linguistic norm as regards the pronunciation of an expression (e.g. /ekinamiks/, /iykinámiks/) count as synonyms, they must be synonyms of a quite different kind from vixen and female fox. For while someone might just conceivably ask "Is / ekinámiks/ the same subject as /iykinámiks/?", no-one, unless he were joking, would give the definition "/ekinámiks/ is /iyktnámiks/". Whereas a quite standard way of defining 'vixen' is to say: "A vixen is a female fox". It seems, therefore, that before one could profitably discuss the 'synonymity criterion' and its intended application, various distinctions would need clarification, including the following.

At least three types of question about meaning may be asked in respect of a given utterance-token. (1) We may be asked to identify the meaning of a given utterance-token in terms of the meaning or meanings assigned to a corresponding utterance-type: e.g. 'Does pink here used of a newspaper mean pink as regards the colour of the paper it is printed on, or pink as regards the political views it expresses?'. (2) We may be asked to identify the meaning of an utterance-token by specifying the paradigm of non-anomalous semantic choices available in the context, and indicating which possibilities are excluded by the occurrence of the token in question. E.g. 'He rang off, replaced the receiver, and walked out of the telephone -.---..' What meanings does kiosk here exclude?' (Answer: kiosk here excludes 'booth', 'room' etc. N.B. The context already excludes 'wire', 'engineer', 'call' and other meanings which might otherwise go with telephone.) (3) We may be further asked to 
specify, in as great detail as may be required, the actual interpretation of that semantic choice, i.e. we may be required to elucidate in full, paying due attention to the speaker, place, time and social setting of the occasion, the implications which the utterance-token would have and have been intended to have - for the audience to which it was addressed. E.g. 'What speed / length of time taken was implied by fast journey in that context?'

It will be relevant to the following discussion to distinguish by referring to questions of types (2) and (3) as questions of tokenmeaning' and questions of type (1) as questions of 'type-meaning classification'. These designations are intended to point to the fact that (1), although ostensibly a question about the meaning of the utterancetoken, is in fact a disguised question about (exemplification of) a type-meaning ${ }^{1}$. This is expedient in order to avoid confusion arising between questions about the meaning of an utterance-token, and questions about the meaning of an utterance-type, which are categorically quite different questions. For to ask about the meaning of an utterance-token is to ask about a particular spatio-temporally unique event; whereas to ask about the meaning of an utterance-type is to ask about an abstraction which may correspond to very many particular events, or to none.

Now Chornsky is insistent that the 'synonymity criterion' must be interpreted as a thesis about the meanings of utterance-tokens. But his

1. Question (1) asks, in effect, 'Which of pink-type's two meanings does this pink-token bear?'. Whereas (2) does not ask 'Which of kiosk-type's two (three...) meanings does this kiosk-token bear?' Nor does (3) ask 'Which of fast-type's two (three..) meanings does this fast-token bear?': for $180 \mathrm{~m} . \mathrm{p.h} . '$ is not a meaning of the English type fast. 
own use of the term synonymity and its cognates appears to cover (and thus fails to differentiate) at least three kinds of equivalence: (a) token-meaning equivalence, (b) type-meaning classification equivalence, and (c) equivalence in meaning of utterance-types ${ }^{2}$. For present purposes this would not matter much if (a) and (b) entailed (c), and likewise 'not (a)' and 'not (b)' both entailed 'not (c)'. But it is important to note that neither set of implications holds 3 .

It might of course be argued that if there were no instances where the meaning of a token $x$ was identical with the meaning of a token $\underline{y}$, then we should have no grounds for calling the corresponding types $X$ and $\underline{Y}$ synonyms at al1. But this is a consideration which cannot be advanced in Chomsky's favour, since the claim he makes is that the existence of certain synonymous expressions in some varieties of English 'falsifies' (sic) the 'synonymity criterion'. But the synonymous expressions cited are types (/ektnámiks/, /iykinámiks/ etc.), whereas the 'synonymity criterion', as already noted, is said to be a thesis about tokens. The assumption that one can argue from synonymity of types to equivalence of meaning of tokens simply begs the question against the defender of

2. E.g. 'there are utterance tokens that are phonemically distinct and identical in meaning (synonyms)' and - in the same paragraph 'such absolute synonyms as/ekinámiks/ and /iykinámiks/.' (Chomsky 1957 p. 95). The former use of synonym seems to cover either (a) or (b), or perhaps both, while the latter corresponds to (c).

3. The token-meaning equivalence of $x$ and $y$ does not entail the synonymity of types $X$ and $Y$, nor vice versa. Similarly, the synonymity of types $\bar{X}$ and $\bar{Y}$ does not entail and is not entailed by the type-meaning $\vec{c}$ lassification equivalence of $x$ and $y$. 
the 'criterion' since, obviously, no grounds have been advanced for claiming that some particular token [ekinámiks] has the same meaning as some other token [iyktnámiks], other than the (alleged) synonymity of the corresponding types. The most that can be made of Chomsky's case on this point would seem to be some such assertion as the following: 'I am inclined to believe that in some varieties of Fnglish instances could be found where a token [ekinámiks] would prove to have no difference in meaning from another token [iykinámiks] .'

But all our attention is being drawn to here is a phenomenon of speech variation. No such instance will 'falsify' the 'synonymity criterion', any more than instances of dialect mixture or bilingualism 'invalidate' the enterprise of phonological analysis. To content otherwise is simply to confuse the notions 'false' and 'inapplicable' 4 . In cases like [ektnámiks]/ [iyktnámiks] the investigator will conclude, if his researches are sufficiently extensive, that one or other pronunciation of the word must be excluded as intrusive. Or, if we insist that both be included within the same phonological description, we must clearly allow him to mark the two pronunciations, along with any similar hesitations for equivalent, eclectic, effectual, etc., as variants. We cannot expect the 'synonymity criterion', or any other proposed phonological criterion, to be capable of consistent application if we suppose the data to include phonologically incompatible realizations

4. Cf. Jones: 1962 \$647: The speech of those whose pronunciation is unstable cannot be reduced to phonemes at all.' This is not an admission that the assumptions of phonology are false, but simply that their applicability is limited. 
of expressions ${ }^{5}$. No linguistic criterion is deemed to hold for whatever data the linguist happens to have in front of him: all that is required is that it hold for all data conforming to a single linguistic norm ${ }^{6}$.

It might be urged on Chomsky's behalf that no such explanation is available for cases like [vikstn] and [fiymeyl faks], supposing that instances could be found where two such tokens did not differ in meaning. But this brings us to a point which raises the question why Chomsky has selected a hypothetical opponent who is not in fact a phonologist at all but a lunatic, i.e. someone who is, apparently, proposing to make a phonological analysis of English with total disregard for any phonetic similarities or dissimilarities between items in the data. One does not wish to defend the views of lunatics. On the other hand,

5. It might perhaps be argued that [ekinámiks] and [iykinámiks] do belong to the same phonological. system in the sense of being statable in terms of different sequences of phonemes drawn from the same (English) inventory. But this is a contingent fact which does not affect the issue: the case is in principle no different from one in which a speaker sometimes does and sometimes does not observe a certain phonemic distinction.

6. There is no presumption that collecting material from one informant guarantees its linguistic uniformity. The irrelevance of Chomsky's objection is indicated by the fact that if the data include speech variations which remain undetected the resultant phonological analysis will be 'wrong' regardless of whether the criteria used are semantic or nonsemantic; e.g. if we ask an informant to pronounce economics on various occasions and, receiving sometimes the answer [ekinámiks] and sometimes the answer [iykinámiks], conclude that [e] and [iy] are members of the same phoneme. Naturally, if the phonologist thinks that by using semantic criteria he will automatically avoid the problem of speech variation he is mistaken. But that is a quite different matter (and a claim which, in any case, no advocate of semantically based phonology makes). 
refuting them is of no great interest?. If the argument is intended as a reductio ad absurdum it fails, since non-semantically based phonology which ignored phonetic similarities and differences would be equally absurd.

Next, it must be observed that Chomsky's claim against the 'criterion' is that it is false both (i) as a necessary condition of phonemic distinctness (against which he adduces synonyms), and also (ii) as a sufficient condition of phonemic distinctness (against which he adduces homonyms). But it would go ill with Chomsky's argument against any sane defender of the 'criterion', since the case against (i) or against (ii) might hold, but just what could not plausibly hold is the simultaneous case against both. To adopt Chomsky's language, the 'criterion' may perhaps be 'false in one direction': precisely what seems unlikely is that it could be shown to be, as Chomsky asserts, 'false in both directions'. This is because, as emerges from the following considerations, at least one part of Chomsky's dual case must be abandoned.

Before any substantive point could be debated between Chomsky and his opponent, they would have to agree on the question: 'Are we concerned with difference of meaning of tokens in identical contexts, or with difference of meaning of tokens irrespective of context?' The answer

7. This would hardly excuse anyone from regarding the lunatic's thesis as one open to refutation by counterexample, which it is not. Qua counterexample, [viksin] / [fiymeyl faks] is just as vain as [ekinámiks] / [i.ykłnámiks] . (It would be equally vain to argue against someone who said "A triangle has four sides" by claiming "I can show you some which have only three".) 
must be that they are concerned with difference of meaning of tokens in identical contexts, since if context is disregarded questions about token-meanings become conflated with questions about type-meanings. The distinction is no longer viable ${ }^{8}$.

Now if context is to be taken into account, the defender of the 'criterion' can claim that the argument from homonymy collapses straight away (since 'I saw him by the bank' i.e. river bank, and 'I saw him by the bank' i.e. First National, patently occur in different contexts: or, should they occur in identical contexts, then the token-meanings do not differ). It is pointless to invoke here the semantic difference 'bank (finance)' Vs. 'bank (river)', since to apply that distinction to tokens in identical contexts is to impose a type-meaning classification, when what is at issue is a token-meaning difference. If, however, someone were to insist that homonyms are nonetheless token-meaning-different in identical contexts, the defender of the 'criterion' would then be justified in maintaining the counterclaim that any expressions are tokenmeaning-different in identical contexts (including synonyms). He does not have to be able to show what the difference is in order to substantiate such a claim: for that cannot be shown either in the case of homonymous tokens in identical contexts.

8. If, for example, we have simply a fragment of undated manuscript bearing just the words milites moriuntur, we are simply not in any position to contrast the token-meaning of these words with the type-meaning of the Latin words milites moriuntur, since the context is unknown. A similar position is reached by disregarding known contexts. 
In short, the argument from homonymy can be pressed, ultimately, only at the expense of sabotaging the argument from synonymy. Likewise, if the argument from synonymy is maintained (by showing lack of token-meaningdifference between items in identical contexts), the argument from homonymy must be abandoned (since there will be parallel lack of tokenmeaning-difference between items in identical contexts).

Chomsky's best move here would be to press the argument from synonymy and drop the argument from homonymy. This means attacking the 'criterion' as a necessary condition of phonemic distinctness, at the expense of conceding it to provide a sufficient condition. But, for reasons to be examined below (2.23), it is precisely the 'criterion' as a necessary condition which is the least indispensable part of any case for a semantically based phonology. Indeed, it was a misrepresentation to contend that it constituted part of that case in the first place (cf. 2.23 n.2).

2.23 A further comnent must be added concerning the question of equivalence (c), i.e. type-meaning equivalence. Here three points should be noticed. First, it is incorrect to claim, as Chomsky does, that the 'synonymity criterion' is properly applicable only to tokens. The phonologist who inquires, for example, whether [pil] and [bil] are phonemically distinct in English is not primarily concerned with tokens that is, with single particular instances of these utterances - but rather with a general question of classification. He begs no questions by distinguishing between a type [pil] and a type [bil], since the types in question are phonetic types. And, in general, one may legitimately 
define an utterance-type in purely phonetic terms without in any way prejudging the question of its phonological status ${ }^{1}$. Second, if the 'synonymity criterion' is interpreted in this sense, i.e. as a thesis about phonetically defined utterance-types, it becomes irrelevant to cite cases of homonymy as weighing against it (since it will not prove possible to assign different meanings to distinct phonetic types of a given homonym.) Ihirdly, granted this interpretation, the 'synonymity criterion' does not in fact represent the position for which advocates of a semantically based phonology have usually argued or need argue. Specifically, it is not usually argued that a phonemic distinction must differentiate meanings (of phonetically defined utterance types) but that such a distinction may differentiate meanings and (sometimes)

1. Various phonetic but non-phonological typologies for utterances might be suggested: cf. Rosetti 1963. In general, the typetoken distinction may be set up in various ways with respect to any particular item. This point may perhaps best be illustrated by analogy. E.g. an English shilling may be regarded as a token of two or more coin-types, according to our interest in coin classification. It may or may not be relevant that it is made of silver alloy, or bears a certain monarch's head, or is valuable by reason of the small number of shillings minted in its particular year. Thus it may be regarded as a token of the type 'English silver shilling', or of the type 'George $V$ shilling', or of the type ' 1930 shilling'. All the relevant characteristics are manifested in praesentia in the token. 
does so in the language under investigation? ${ }^{2}$. To bring up such examples as English economics, adult, etc. is thus to miss the point, since a semantically based phonological analysis of Inglish will have no difficulty in establishing that in (many) other instances, the phonetic differences in question do distinguish meanings, and hence a correct phonological analysis of these differences will result. 2.24 A semantically based phonology is compatible with the denial of synonymy, provided phonological units can be established without exclusive reliance on controversial cases. The Bloomfieldian position ${ }^{1}$ with regard to such pairs as /ekinámiks/ and /iykinámiks/ may be represented as follows: 'I assume that the two differ in meaning, although I am unable to ascertain what the difference is.' 'This may be an unprofitable assumption for purposes of semantic analysis; but that is not the

2. Fischer-Jørgensen 1956 p. 144: 'it is only required that a replacement should be capable of entailing a difference in the content, not that it should always do so.' Chomsky's formulation of the 'synonymity criterion' makes the exror of representing the condition of meaning-difference as a necessary condition as well as a sufficient condition. Chomsky is not the only opponent of semantically based phonology guilty of this misrepresentation. Cf. Bloch 1948 32.2: IIt is customary to base the difference between distinctive and non-distinctive sounds on meaning: to say, for instance that any interchange of distinctive sounds will affect the meaning of a word or phrase, while any interchange of nondistinctive sounds will leave the meaning unaffected.' It is ironical that Bloch should refer for confirmation of this statement to Bloch and Prager 19423.1 , where, however, the promised confirmation is not forthcoming. The position stated in Bloch and Trager 19423.1 coincides in fact with that of Fischer-Jørgensen (above): distinctive differences or contrasts are there said to be 'capable of distinguishing one meaning from another', but there is no claim that they must invariably do so.

1. C.f. 1.3 . 
question here. The issue between a Bloomfieldian phonologist and Chomsky must be whether or not such an assumption is consonant with a correct phonological analysis of English. If indeed all the pairs exhibiting the same phonetic differences as [ekinámiks] and [iykinámiks] were controversial cases, in the sense that no semantic distinction between the members of such pairs could be clearly established, a Bloomfieldian phonologist would have no ground for recognizing the distinction as phonological. But he is not committed to the proposition that failure to discover a semantic difference in one such case is sufficient to justify classing the members of that pair as phonemically identical. Furthermore, for the Bloomfieldian it is only on condition that a semantic difference can be established for at least one such case that any question of assuming that /ekinámiks/ and /iyktnámiks/ differ in meaning arises.

2.25 Finally, the theoretical obscurity of Chomsky's own position must be noted. It is far from clear why anyone should regard the "pair test" in the form described by Chomsky as a test of phonemic distinctness, when what it is is manifestly something quite different, namely a test of auditory distinguishability of utterances. Such a test would, of course, become a test of phonemic distinctness if we had some guarantee that the utterance-tokens in question were not distinguishable by any other than phonemically relevant features. But any such proviso must of necessity involve the phonologist in circularity.

Nor can this circularity be broken by the proposal to define the phoneme simply by reference to the "pair test": e.g. by saying 'Let us 
mean by phonemically distinct nothing more than distinct as measured by the pair test.' For the effect of such a proposal would be simply to equate 'phonological unit' with 'auditorily distinguishable unit', and for purposes of phonological analysis this equation is clearly intolerable.

2.26 To summarise, Chomsky's arguments, considered as an attack upon semantically based phonology, are misdirected inasmuch as the positions attributed to his opponents are not, on the whole, those which semantically based phonologists have defended, nor in any case those which they need defend. As a positive phonological programme, on the other hand, Chomsky's appears to lack precisely the theoretical foundations required to validate an independent branch of scientific inquiry. With regard to synonymy, his thesis reduces to the trivial truth that if there are phonologically different expressions with the same meaning, sameness of meaning is not a sufficient condition of phonological identity. 2.3 It will at this point be appropriate to examine a divergence of opinion among semantically based phonologists as to the scope of phonology.

2.31 The fact that in any given language certain utterances do, while others do not, have the same meaning, is fundamental to the distinction between phonology and phonetics as drawn by Trubetzkoy:

'Die Phonologie hat zu untersuchen, welche Lautunterschiede in der betreffenden Sprache mit Bedeutungsunterschieden verbunden sind, wie sich die Unterschiedungselemente (oder Male) zueinander verhalten und nach welchen Regeln sie miteinander zu Wörtern (bezw. Sätøen) kombiniert werden dürfen'. ${ }^{1}$ 
A characteristic feature of phonetics is its lack of concern with meanings:

Hesonders kennzeichnend für die Phonetik ist die vollkommene

Ausschaltung jeder Beziehung zur sprachlichen Bedeutung der untersuchten Lautkomplexe'. 2

Whereas the basis of phonological description is the discovery that certain phonetic contrasts differentiate meanings:

'Der Anfang jeder phonologischen Beschreibung besteht in der Aufdeckung der in der betreffenden Sprache bestehenden bedeutungsdifferenzierenden Schallgegensätze' ${ }^{3}$

2.32 The notion 'capacity to differentiate meanings' thus enters into the definition of certain basic phonological concepts in Trubetzkoy's theory, notably that of 'phonological opposition' ('phonologische Opposition'), which Trubetzkoy introduces as follows:

'Schallgegensätze, die in der betreffenden Sprache die intellektuelle Bedeutung zweier Wörter differenzieren können, nennen wir phonologische (oder phonologisch distinktive oder auch distinktive) Oppositionen'. I

The same applies to Trubetzkoy's concept of the phoneme, which is derived from that of phonological opposition, via that of 'phonological unit': 'Unter (direkt oder indirekt ${ }^{2}$ ) phonologischer Opposition verstehen

2. Trubetzkoy 1939, p. 13.

3. Trubetzkoy 1939, p. 17.

1. Trubetzkoy 1939, p. 30 .

2. The distinction between direct and indirect phonological oppositions is that the terms of indirect oppositions occur in complementary distribution but share no common phonetic features distinguishing them in the sound system of the language, whereas the terms of direct oppositions are not in complementary distribution (Trubetzkoy 1939, p. 32). 
wir also jeden Schallgegensatz, der in der gegebenen Sprache eine intellektuelle Bedeutung differenziexen kann. Jedes Glied einer solchen Opposition nennen wir phonologische (bezw. distinktive) Einheit' 3

'Phonologische Einheiten, die sich vom Standpunkt der betreffenden Sprache nicht in noch kürzere aufeinanderfolgende phonologische Einheiten zerlegen lassen, nennen wir Phoneme'. 4 The phoneme is thus essentially a meaning-distinguishing unit. 5 2.33 If, then, a language has a given distinction $D$ and a set of pairs $\underline{a}_{1}$ vs. $\underline{b}_{1}, \underline{a}_{2}$ vs. $\underline{b}_{2}, \underline{a}_{3}$ vs. $\underline{b}_{3} \ldots$ such that both members of each pair are realizations of meaningful expressions, and each pair is differentiated solely by the distinction D (as e.g. English [ $p^{h}$ in] vs. [bin], $\left[p^{h} i: k\right]$ vs. $[b i: k],\left[p^{h} æ t\right]$ vs. $[b æ t] \ldots$ where $\underline{D}=\left[p^{h}\right]$ vs. $\left.[b]\right)$, it follows from the definitions cited in 2.32 that $\underline{D}$ will constitute a phonological opposition if and only if for at least one pair a vs. $\underline{b}$ the expressions realized by $\underline{a}$ and $\underline{b}$ differ in meaning. For if this is not the case then $\underline{D}$ cannot be held to differentiate meanings. But since $\underline{a}$ and $\underline{b}$ cannot differ in meaning if they are realizations of synonymous expressions, it is a necessary condition that the expressions realized by some pair $\underline{a}$ and $\underline{b}$ should not be synonymous. Strictly, since the meanings Trubetzkoy specifies are 'intellectual meanings' (intellektuelle

3. Trubetzkoy 1939, pp. 32-33.

4. Trubetzkoy 1939, p. 34.

5. The credit for being the first to advance this concept of the phoneme is given by Trubetzkoy to L. V. Ščerba (Trubetzkoy 1939, p. 34 n.I). 
Bedeutung) the relationship in question between the expressions realized by $\underline{a}$ and $\underline{b}$ will be that of 'intellectual synonymy'. Thus, for every case in which $\underline{D}$ has the status of a phonological opposition, there must be at least one pair $\underline{a}$ and $\underline{b}$ which do not realize 'intellectually synonymous' expressions (although there may be other pairs in the same set which do). For every case in which $\underline{D}$ does not have the status of a phonological opposition, no pair in the set will realize 'intellectually synonymous' expressions. Thus determining the phonological status of $\underline{D}$ is tantamount to determining whether or not the relationship of 'intellectual synonymy' holds between expressions realized by the members of some pair $\underline{a}$ and $\underline{b}$ in the set. 2.34 The significance of the term 'intellectual meaning' (2.33) derives from Trubetzkoy's acceptance of a distinction between phonology and phonostylistics, based on Bühler's analysis of the three functions of speech ${ }^{1}$. Phonology, in Trubetzkoy's view, is concerned only with the 'intellectual meanings' of expressions, that is to say with the 'representative' function of speech. All phonetic distinctions which serve the 'expressive' and 'appellative' functions are the concern of phonostylistics, not phonology. Thus from the phonological point of view any two expressions $\underline{a}$ and $\underline{b}$ may be considered synonymous if they differ in respect of the expressive or appellative functions, provided that they do not differ in respect of the representative function (whatever that is taken to be in a given case). E.g. if it is agreed that a

1. Trubetzkoy 1939, pp. 17-29. 
and $\underline{b}$ have different meanings, but that the difference of meaning consists solely in that a expresses emotional involvement on the part of the speaker, whereas $\underline{b}$ is emotionally 'neutral' (cf. French [e'ppuvätabl] vs. [epuvã'tabl] ), this difference of meaning does not count against the assessment of $\underline{a}$ and $\underline{b}$ as 'intellectual synonyms.'

2.35 The importance of the function of distinguishing between 'intellectual meanings' is further evident in the rules given by Trubetzkoy for the determination of phonemes. The first rule is as follows:

'Wenn zwei Laute derselben Sprache genau in derselben lautlichen Umgebung vorkommen und mit einander vertauscht werden dürfen, ohne dabei einen Unterschied in der intellektuellen Wortbedeutung hervorzurufen, so sind diese zwei Laute nur fakultative phonetische Varianten eines einzigen Phonems ${ }^{1}$.

This rule is a statement of a sufficient condition for classifying the distinction between sound $\underline{\mathrm{a}}$ and sound $\underline{\mathrm{b}}$ as non-phonemic. By implication it states also a sufficient condition for refusing to classify two phonetically distinct meaningful items as realizations of synonymous expressions (i.e. if the two items differ solely in respect of sounds which always occur in the same environments and may always be interchanged without altering the 'intellectual meanings' in question, then the two items are formally (phonologically) identical, and hence there is no question of synonymity.)

1. Trubetzkoy 1939, p. 42. 
Irubetzkoy does not always, however, take the existence of synonyms sufficiently into account, as in the formulation of the following rule:

'Wenn zwei Laute genau in derselben Lautstellung vorkommen und nicht mit einander vertauscht werden können, ohne dass sich dabei die Bedeutung der Wörter verändern oder das Wort unkenntlich werden würde, so sind diese zwei Laute phonetische Realisationen zweier versichiedener Phoneme' ${ }^{2}$.

This rule states a sufficient condition for classifying the distinction between sound $\underline{a}$ and sound $\underline{b}$ as phonemic. But it falls foul of cases of the following kind. Suppose we are investigating the [t]/ [d] distinction in a language in which all words are monosyllabics having the structure CV. We find that for some values of $\mathrm{V}$ there corresponds to [tV] a word pronounced [dV]with a different 'intellectual meaning'. In other cases there corresponds to $[t V]$ no word * [dV]. But in a third class of cases there corresponds to $[\mathrm{tV}]$ a synonym pronounced $[\mathrm{dV}]$. Under Trubetzkoy's rule cited above, the $[t] /[d]$ distinction fails in this language to satisfy the sufficient condition for classification as a phonemic opposition. Yet, ex hypothesi, it is a distinction capable of distinguishing between 'intellectual meanings'. It must be assumed that Trubetzkoy either overlooked the possibility of such cases in formulating his rule, or else believed that they never occurred. To take such cases into account, the rule would need to be amended by adding some such proviso as 'or substitute for one word its intellectual synonym'.

2. Trubetzkoy 1939, p. 44. 
2.36 The scope of phonology according to Trubetzkoy is more restricted than in the view of those linguists (e.g. Bloomfield) who draw no distinction between phonology and phonostylistics. The distinction between phonology and phonetics, on the other hand, is drawn very similarly in both cases. According to Bloomfield:

'The study of language can be conducted without special assumptions only so long as we pay no attention to the meaning of what is spoken. This phase of language study is known as phonetics ${ }^{1}$... The study of significant speech-sounds is phonology or practical phonetics, Phonology involves the consideration of meanings' ${ }^{2}$. Like Trubetzkoy, Bloomfield distinguishes between distinctive and nondistinctive sound-features, and assigns the study of the former to phonology:

The features of sound in any utterance, as they might be recorded in the laboratory, are the gross acoustic features of this utterance. Part of the gross acoustic features are indifferent (non-distinctive), and only a part are connected with meanings and essential to communication (distinctive) ${ }^{3}$.

Bloomfield further states:

'It is important to remember that practical phonetics and phonology presuppose a knowledge of meanings: without this knowledge we

1. Bloomfield 1935, p. 75 .

2. Bloomfield 1935, p. 78 .

3. Bloomfield 1935, p. 77. 
could not ascertain the phonemic features ${ }^{4}$

But the meanings relevant to a Bloomfieldian analysis are not the 'intellectual meanings' of Trubetzkoy. Bloomfield's definition of meaning is a very broad one: 'the meaning of a linguistic form (is) the situation in which the speaker utters it and the response which it calls forth in the hearer'. ${ }^{5}$ Bloomfield further specifies that 'a very important part of every situation is the state of the speaker's body' which includes 'the predisposition of his nervous system, which results from all his experiences, linguistic and other'6. It appears from this that the meaning of an expression for Bloomfield encompasses both what Trubetzkoy terms its 'intellectual meaning' and also elements which belong to the 'expressive' and 'appellative' functions of speech. While admitting the difficulty of obtaining reliable data about meanings 7 , Bloomfield nonetheless insists on the importance of information about meanings as a prerequisite of phonological analysis. 'Since we can recognize the distinctive features of an utterance only when we know the meaning, we cannot identify them on the plane of pure phonetics' 8 . 'The observer who hears a strange language, notices those of the gross acoustic features which represent phonemes in his own language ox in other languages he has studied, but he has no way of knowing whether these features are significant in the language he is observing ${ }^{9}$. However, it is not

4. Bloomfield 1935, pp. 137-138.

5. Bloomfield 1935, p. 139.

6. Bloomfield 1935, p. 141.

7. Bloomfield 1935, pp. 139m142.

8. Bloomfield 1935, p. 77 .

9. Bloomfield 1935, p. 93. 
necessary to start with a comprehensive account of the meaning of every utterance under investigation: what is needed to discover the phonemic distinctions is sufficient information to determine 'which utterances are alike in meaning, and which are different ${ }^{10}$.

2.37 Thus Bloomfield places the phonologist in essentially the same position as Trubetzkoy: he is given sets of pairs of phonetically different items and must determine which pairs are realizations of expressions differing in meaning, and which are not. Since, however, Bloomfield explicitly rejects the view that natural languages have synonyms as untenable for purposes of linguistic analysis ${ }^{1}$, it follows that the phonologist's task is to assign phonetically different pairs to one or other of two classes; namely, pairs which are and pairs which are not variant realizations of one and the same phonological form. But Bloomfield's broader view of the scope of phonology means that it will be sufficient for the phonologist to discover any consistent difference between the situations in which members of a given pair are uttered in order to claim that there is a difference of meaning and thus of (phonological) form. On this view, if it can be established that French [epuvãtabl] is emotionally 'neutral' whereas [e'ppuvätabl] is emotionally 'committed', then /epuvấtabl/ and /e'ppuvátabl/are different non-synonymous expressions.

10. Bloomfield 1935, p. 93.

1. Bloomfield 1935, p. 145. Cf. above 1.3 
2.38 A semantically based phonology is, then, compatible with somewhat varying criteria for synonymy. According to one view, it is possible that two phonetically different types may be realizations of different synonymous expressions, although conveying different 'phonostylistic' information. According to another view, any systematic correlation between two different phonetic types and two different situational features or sets of features counts against the classification of those types as realizations of different synonymous expressions. And 'in between' these views one can envisage others, the criteria for synonymy varying according as the scope of phonology tends to be 'restricted' (Trubetzkoyan) or 'unrestricted' (Bloomfieldian). We may speak, then, of synonymy as related to the assumed scope of phonological investigations in any given case. Accordingly, the statement that $\underline{\mathrm{a}}$ and $\underline{\mathrm{b}}$ are synonymous may or may not imply the absence of distinctions of an expressive or appellative nature. In every case, however, the phonologist's concern with meanings ends with his postulating a sameness or difference of meaning between certain expressions. He is not further concerned with any 'positive' characterization of the meaning(s) in question, nor with justifying his postulation other than by reference to the analytic procedures which, qua phonologist, he employs. Such procedures must now be considered in some detail, since upon the nature of the procedures depends the question whether a clear operational crtiexion for synonymity can be given, or whether the semantically based phonologist is reduced to borrowing (with or without acknowledgment) from some (specified or unspecified) prior semantic theory. 
2.4 It must first be noted that the claim that phonemic distinctions, by definition, distinguish meanings does not commit the claimant to the view that establishing a phonemic distinction between $\underline{a}$ and $\underline{b}$ is dependent on the prior discovery of a difference in meaning between a and $\underline{b}$. This latter view has, however, been independently maintained. A well known article by Pike ${ }^{l}$ contends that ascertainment of certain semantic information is essential for the determination of phonemes: 'The phonemic analysis cannot be completed until some initial grammatical steps are taken. Perhaps the most important of these is the identification of at least a limited number of morphemes. Thus Hockett, and Bloch and Trager utilize pairs of utterances which (1) have different meanings, and (2) are minimally different in their pronunciation.

Recognizing that the utterances are different in meaning is a grammatical process, not a phonemic one. It is thus absolutely essential that a minimal grammatical identification be achieved before phonemic analysis can be carried on: the irreducible minimum prerequisite is that the investigator know enough about two items to be sure that they are 'different''.

But if we must discover differences in meaning before we can establish phonemic distinctness, then since we cannot discover differences in meaning if we do not know what differences in meaning are, phonology becomes dependent upon a prior theory of meaning, or at least upon

1. Pike 1947, p. 158 . 
the prior acceptance of some partial theory of meaning - that part which deals with differences of meaning and thus with non-synonyms. But since synonymy and non-synonymy are heads and tails of the same coin, a theory of synonymy then becomes a prerequisite of phonological analysis. Some critics ${ }^{2}$ of semantically based phonology evidently believe that appeal to meanings in phonological definitions and analytical. procedures renders this conclusion inevitable, and, seeing no well established prior theory of synonymy available, reject semantically based phonology for this reason. Nor are matters improved by the fact that some semantically based phonologists commit the theoretical error of equating the discovery of differences in meaning with the discovery of informants' beliefs about differences in meaning. Or, if not equating the two, they are at least content to treat the former as directIy derivable from the latter. Thus, for example, Fischer-Jórgensen contends that the necessary information can be obtained by the phonologist by 'eliciting short utterances and presenting them to a native who has to decide whether their meaning is the same or different ${ }^{3}$. Specifically, the native speaker merely has to say 'yes' or 'no' to a direct question of the form 'Does a mean the same as b?' Fischer-Jфrgensen goes to some lengths to justify this procedure and to explain why a simple 'yes/no' answer is adequate. The reason is that in the direct question 'the utterances are taken out of their natural (Iinguistic and non-linguistic) context. Consequently,

2. ..g. Chomsky, Cf. 2.1.

3. Fischer-J фrgensen 1956, p. 142. 
these utterances have no contextual differences of meaning, and the native will react to them as representatives of classes of utterances and will react to their meanings as being the same (in the sense of representatives of one class of meaning) or different (in the sense of representatives of different classes of meaning). In most cases it is possible to leave this decision of being the same or different to the informant, simply because the relation between sound and meaning in the linguistic sign is arbitrary and unsystematic, so that it is rare that small differences of sound (which will be the crucial ones for the investigator) correspond to small and dubious differences of meaning, whereas it will normally happen that they correspond to obvious differences of meaning .... 4

It appears from this that the linguist, in Fischer-Jørgensen's view, is engaged in the enterprise of discovering the proclaimed semantic beliefs ${ }^{5}$ of speakers, in order to differentiate thereby phonemically distinct from non-phonemically distinct utterances. This is confirmed by the following revealing comment:

'Of course, if the linguist himself masters the language,

4. Fischer-Jørgensen 1956, p. 142. Fischer-Jprgensen concedes that there may be borderline cases 'where gradual differences of sound will correspond to gradual differences of meaning'.

5. Examples of statements which proclaim semantic beliefs are: (i) 'To disembark' means ' to go on shore from a ship', (ii) 'Rejection' does not mean the same as 'refusal', (iii) There is no French word which means the same as 'chair', (iv) 'Slithy toves' doesn't mean anything in English. 
the problem of avoiding meaninganalysis is of no practical importance ${ }^{6}$.

The implication is, clearly, that if the linguist speaks the language himself, then simple introspection will tell him which utterances differ in meaning and which do not. In other cases, where informants can be interrogated, it would seem that semantic distinctness - and hence phonemic distinctness - is established directly by informants' responses to questions about sameness of meaning.

Such a view is unacceptable as a basis for phonology, for at least two reasons. First of all, phonology cannot, any more than any other branch of linguistics, afford to ignore the distinction between fact and belief. Just as in grammar a distinction must be drawn between granmatical sentences and sentences which informants are willing to call 'grammatical', so here there must be no confusion between a difference of meaning and what informants are willing to call a 'difference of meaning'. Equally, in the case where the linguist is his own informant, there must be no equating the ascertainment of semantic facts with introspection. By introspection, the linguist merely substitutes his own semantic beliefs for those of an informant - and this will not do, if linguistic analysis is to be counted as different from autobiography. Second, even if semantic beliefs as proclaimed by speakers were held to reflect certain facts about meaning in a reliable way ${ }^{7}$, it is

6. Fischer-Jørgensen 1956, p. 142 .

7. This, of course, could never be established if we refused to make the distinction between semantic facts and semantic beliefs in the first place. 
clear that the naive pre-theoretical concept of 'meaning' appealed to in the question 'Does a mean the same as $\underline{b} ?$ ' - as put to an unsophisticated informant - is simply not the concept of meaning in which the linguist is interested (any more than the naive pre-theoretical concept of a 'sound' corresponds to the linguist's 'phoneme': one might as well go about phonological analysis by asking informants directly 'Would you say these sounds are the same?'). But since, into the bargain, we have no guarantee that semantic beliefs - either in general or in particular instances - are based upon some uniform concept of meaning which language-users happen to have, exactly what it is that has been established when informants agree verbally upon (or when the linguist is himself introspectively sure of) a 'difference of meaning' is itself obscure. Nor can it be argued that at least the linguist's own introspections are exempt from this obscurity. For to the extent that he is able to give some account of what it means to assert or deny sameness of meaning he is acting as his own analyst, not his own informant. The fact that the semantic beliefs under analysis are his own becomes at that point irrelevant.

2.41 The attempt to base phonological analysis upon direct establishment of the proclaimed semantic beliefs of native speakers destroys a parallelism which it is important to preserve between that side of the analysis which deals with sounds and that side which deals with meanings. The semantically based phonologist should start on the one hand from certain observable phonetic differences, and on the other from certain observable differences of usage. It will be his ultimate objective to 
determine how these may best be accounted for (i) by postulating phonemic differences, and (ii) by postulating differences in meaning. But are these two objectives attainable independentily? If (i) is regarded as attainable independently of (ii), then the case for a semantically based phonology collapses. If $(i, i)$ is regarded as attainable independently of (i), it becomes incumbent upon the theoretician of a semantically based phonology to set out and justify the semantic theory which guarantees his procedures and conclusions under (ii). But in fact he usually omits to do this, starting his discussion at a point where it is assumed that one already has a fund of semantic information available $e^{2}$.

1. Trubetzkoy is a notable example. Another is Twaddell, who attempts to bypass the problem as follows:

The events which correspond to the 'same' form are phonetically significantly alike, for by 'significant' we indicate the correlation of an utterance to a social situation. The phonetic events 'Light the lamp' as produced by two different individuals are objectively very different; in so far as these events evoke similar responses in similar social situations, the two events are phonetically significantly alike.' (Twaddell $1935 \mathrm{p}$. 4l). But taken literally this position allows for indefinitely many degrees of phonemic distinctness, since different events, different social situations and different responses will always be similar up to a point, but up to a different point in different cases. To say that the phonologist recognizes formal identity 'in so far as' events have certain similarities dispenses from the obligation to specify the semantic conditions further; but 'in so far as' wins this dispensation at the cost of opening a Pandora's box full of disparate similarities.

2. It is undoubtedly this, in part at least, which has led Chomsky and others to view semantically based phonology with scepticism. One cannot take seriously a claim to the effect that phonemes distinguish meanings, when it rests merely upon a procedural assumption which no-one ever justifies. 
There is, however, a third possibility, which allows the interdependent attainment of both objectives. The account which follows $(2.42-2.43)$ argues that this third passibility is theoretically and practically viable, and that a set of procedures is available which begs none of those questions which are indeed begged if we suppose that the phonologist has available some separately acquired fund of semantic information. This argument, if successful, affords the semantically based phonologist a stance which is not vulnerable to the objections previously discussed. It will also be argued that one can arrive in this way at a criterion of synonymity which is adequate in the context of phonological analysis but which is not derived from a prior theory of the meanings of linguistic expressions. 2.42 Let the phonologist begin by establishing that there is a nonphonorelative difference of usage ${ }^{l}$ between a phonetic type a and some grossly dissimilax phonetic type $\underline{b}$. This difference of usage is estab-

1. A difference of usage is established by finding correlations of the following kind which hold for all the data under observation. Let ap be the proposition that for all the a-tokens, an a-token occurs if and only if $p$, and pa the proposition that iff $\bar{p}$ then no a-token occurs. Similarly for $\underline{b}$ and $\mathrm{q}$ the propositions $\mathrm{bq}$ and $\mathrm{qb}$. For $\underline{a}, \underline{b}, \underline{p}$ and $q$ togethe $\bar{r}$, we have eight such propositions: $\mathrm{ap}, \underline{\mathrm{pa}}, \underline{\mathrm{aq}}, \mathrm{qa}, \underline{\mathrm{bp}}, \mathrm{pb}, \underline{\mathrm{bq}}$ and $\mathrm{qb}$. Then a difference of usage between the phonetic types a and $b$ is defined as any difference which can be stated in a conjunction of the following form: ap. $\underline{\mathrm{bq}}$. qa. pb. Since the satisfaction of such a conjunction depends simply on establishing the truth values for the component propositions, we in no way prejudge the question whether items for which a difference of usage is established are realizations of expressions having the same meaning or different meanings. A difference of usage is phonorelative if $\underline{p}$ or $\underline{q}$ include reference to phonic features of $\underline{a}$ or $\underline{b}$, or of the contexts in which they occur. 
lished without prejudice to the question whether it will ultimately be accounted for in terms of a difference in meaning. For example, if the phonologist's informants are willing to apply a-tokens to members of a certain class of objects $\underline{a}$ but reject their application to members of a disjoint class of objects $\mathcal{Q}$, but, conversely, apply b-tokens to objects of class $\hat{q}$, while rejecting their application to members of class $\underline{\alpha}$, such tests could reasonably be taken as establishing a difference of usage between $\underline{a}$ and $\underline{b}$. The phonologist may then bring forward a phonetic type '' having some minor point of phonetic difference with $\underline{b}$, in order to determine whether or not $\underline{b}$ and $\underline{b}$ ' are phonologically distinct. The tests with $\underline{x-o b j e c t s}$ and $\beta$-objects may be repeated, substituting $\underline{\mathrm{b}}$ ' for $\underline{\mathrm{b}}$. If exactly the same results ensue ${ }^{2}$, the phonologist may then formulate a provisional hypothesis (H) as follows:

$\mathrm{H}$ 'Either (i) $\underline{b}$ and $\underline{b}$ ' do not contrast phonologically, or (ii) $\underline{b}$ and $\underline{b}$ ' are realizations of synonymous expressions.' He must then test $H$ by attempting to discover values of $\underline{p}$ and $\underline{q}$ for which the conjunction $\underline{b p}$. $\mathrm{b}^{\prime} \mathrm{q} \cdot \underline{\mathrm{gb}}$. $\mathrm{pb}^{\prime}$ issatisfied. On failure to discover appropriate values for $\underline{p}$ and $\underline{q}, H$ may be considered established. 2.43 The next step is to determine which of the alternatives $H(i)$ or H (ii) is preferable. This may be done as follows. Let the phonetic difference between $\underline{b}$ and $\underline{b}$ ' be called $\underline{D}$. Further pairs of items $\left(\underline{k}-\underline{k}^{\prime}, \underline{I}-\underline{I}^{\prime} \cdot.\right)$, each pair differing solely by $\underline{\mathrm{D}}$, must be examined ${ }^{\mathrm{I}}$.

2. i.e. if $\underline{a p} \cdot \underline{b q} \cdot \underline{q a} \cdot \underline{p b} \cdot \underline{b^{\prime} q} \cdot \underline{p b^{\prime}}$ with values of $\mathrm{p}$ and $q$ held constant, allowing for the substitution of $\underline{b}$ ' for $\underline{b}$.

1. The problem of defining $D$ is for present purposes irrelevant: i.e. whichever value(s) of $\underline{D}$ the phonologist decides to investigate, the procedures here described are valid. 
Wach pair will be tested against other phonetic types $(\underline{\mathbf{r}}, \underline{\mathbf{s}} \ldots)$ when a difference of usage can be demonstrated between such a type and either member of the pair. These tests will show whether or not the hypothesis $\mathrm{H}$ can be established for $\underline{k} \underline{k}^{\prime}$ ( $i . e$. that $\underline{k}$ and $\underline{k}^{\prime}$ either (i) do not contrast phonologically, or (ii) realize synonymous expressions). By testing $\underline{k}-\underline{k}^{\prime}, \underline{I}-\underline{I}^{\prime}$... the phonologist can establish a matrix of the values of $\underline{D}$ for a number of phonetic pairs. This matrix will show the regularity with which $\mathrm{D}$ is associated with differences of usage for various phonetic types in the language. Such a matrix must be interpreted by the phonologist in the light of the following two methodological principles:

1. Any assignment of phonological status which has the effect of proliferating synonyms in the lexicon is suspect.

2. Any such assignment which creates a substantial class of synonym pairs in the lexicon which may be subsumed under a general rule of form $F$, where $F$ is 'Tor every lexical entry having the phonological characteristic(s) $\mathrm{Ni}$ there is a synonymous entry having the phonological characteristic(s) $\underline{N}^{\prime}$ must be rejected ${ }^{2}$.

2. Two explanatory comments on Principle 2 may be offered at this point. First, the provision 'a substantial class of synonym pairs' takes care of the kind of objection raised by tbeling to the employment of semantic criteria to determine phonological. differences: 
'Usually linguists refer

in this connection to meaning. But this cannot be done consistently. An example may illustrate this: the Russian words skkaf and škap are usually regarded as synonyms. If we base, however, our criterion for the distinction between relevant and nonrelevant differences of sound upon meaning, we must admit that in Russian immediately after ška- the opposition stop versus continuant is neutralized in contact with labiality and orality. Nevertheless, nobody seems to accept this formulation. To every Russian [škaf] and [škap] are no doubt two different forms. This criterion should prevail everywhere as it does in this case.

'I take an Finglish example from lockett: root may be pronounced [ruwt] or [rut]. In such cases the environment, ska- or $\underline{r-t}$, is usually considered too complicated or too specific for the assumption of a neutralizing influence, but this can only mean that probably a native speaker will not be inclined to interpret the forms as the same: we can in no way dispense with an appeal to his interpretation'. (Ebeling 1960 p. 38).

Evidently, the exact number which counts as a 'substantial class' is open to argument; but it is nonetheless a different way of deciding the issue from appealing to the native speaker's Sprachgefühl. To reject škaf and škap as an example of neutralization because it would be a unique example is in no sense equivalent to admitting škaf and škap as synonyms because that is how Russian speakers feel about the matter.

Second, there will doubtless be cases in which the admission of two sounds as belonging to different phonemes creates a substantial class of synonym pairs, even though that solution is imposed by other examples in which a difference of usage can be shown to depend solely on the distinction between the sounds in question. (Such cases may even be common in a language where the phonological system is undergoing a change at certain points). It will be the duty of the phonologist to draw attention to such 'inconsistencies' in the way the language utilizes sound differences; but the validity of the principle invoked above is in no way called in question by these cases. 
In short, the phonological status of $\underline{D}$ will be decided in such a way as to comply with the rejection of $H$ (ii) wherever possible. 2.44 The justification for the two methodological principles mentioned above is obvious, for the principles themselves are essentially no different from those implicit in the application of such phonological concepts as 'free variation' and 'neutralization'. There is in semantically based phonology a close connexion between these concepts and that of 'synonymy.' 'Free variation' and 'neutralization' may both, from a certain viewpoint, be seen as safeguards against the proliferation of synonyms. For example, if a linguist investigates the hypothesis that a language has the phonemic oppositions /p/ vs. /b/, /t/ vs. /d/ and $/ \mathrm{k} /$ vs. $/ g /$, and finds that as regards the /p/ vs. /b/ opposition (but not the other two) all pairs of items differentiated solely by that opposition show no difference of usage, this will be a reason in favour of modifying the hypothesis by treating [p] and [b] as free variants ${ }^{1}$. By this revision the provisional phoneme inventory is reduced by one, and the provisional lexicon is consequently alleviated by the removal of a set of potential synonym pairs involving simply the /p/ vs. /b/ distinction, each such pair being now replaceable by a single entry. Similarly, in such a language as Spanish we have the choice between accepting a great proliferation of synonym pairs of the type /ganar/ 'to gain': /ganar/ 'to gain', and saying that the opposition/r/ vs.

1. Assuming there are no features of the distribution of [p] and [b] which argue against their treatment as free variants. 
$/ s /$ is neutralized in final position. The reason why it is preferable to accept the latter alternative is twofold. First, back door admissions make nonsense of front door refusals. By adhering rigorously to the principle 'one a phoneme always a phoneme' we would simply be achieving a certain 'front door' uniformity of phonological description at the expense of a very great 'back door' multiplication of lexical entries for Spanish. Second, the principle itself is brought in disrepute if the class of candidates admitted at the back door contains all and only those refused at the front. The possibility in this hypothetically expanded Spanish lexicon of subsuming the relevant synonym pairs under a general rule of form $F$ (i.e. 'for every lexical entry ending in / $r$ / there is a corresponding synonym ending in $/-s / 1$ ) shows that we are dealing with a fact which should properly be accounted for at the level of phonology. In short, a semantically based phonology may be said to operate under the standing prohibition: synonyma non multiplicanda praeter necessitatam. 2.45 On this view, the 'sameness of meaning' which the phonologist postulates reduces solely and simply to failure to meet certain criteria of difference of usage as evidenced by test procedures employed. It remains to stipulate two general conditions concerning non-phonorelative differences of usage in phonology.

The first condition is that the differences of usage which the phonologist establishes in investigating the phonological status of a given phonetic difference or set of differences must show 'typological. similarity'. 'This condition is simply a safeguard against conflating in one and the same analysis facts which properly belong to separate analyses. 
The condition would be infringed, for example, if the phonologist admitted simultaneously as evidence bearing on the phonological status of one distinction both (i) a difference in usage between $\underline{a}$ and $\underline{b}$ which consisted in their use by speakers of different regional provenance and (ii) a difference in usage between $\underline{\mathrm{c}}$ and $\underline{\mathrm{d}}$ which consisted in their application by speakers of whatever regional provenance to different objects. Two such differences show too great a typological dissimilarity to be reliably included in the same analysis ${ }^{1}$.

The second condition is that the differences of usage must be 'informational': i.e. we assume that no conventional ${ }^{2}$ correlation between phonetically differing items and different usages is the concern of the linguist unless there is evidence to show that speakers are aware of the correlation. This condition, in appearance trivial, is in fact not so, since it can be shown that some accounts of linguistic analysis, e.g. Bloomfield's, fail to satisfy it. It would, for example, be sufficient in principle for a Bloomfieldian linguist to establish

1. It is easy to exemplify relatively gross infringements of the condition; but no detailed typological theory of usage seems to have been worked out by linguists. Conceivably, there might be relevant phonological differences within the 'representative' function of speech which Trubetzkoy correlates with the province of phonology: e.g. there might be, to adopt Malinowski's distincttions, a phonology of pragmatic utterances, a phonology of narrative, and a phonology of phatic communion - at least for certain languages. Similarly, there might be some point in distinguishing a phonology of statements, a phonology of questions, a phonology of exclamations, and so on. Such matters have not been at all fully explored.

2. i.e. non-natural. Phonology has no concern with phonic differences attributable to natural causes (e.g. age, sex, physiognomy). 
any consistent correlation between two phonetically different items and two different situational features in order to conclude that the expressions in question differed in meaning (2.37). The difference in usage need not, in Bloomfield's formulation, be informational. But if speakers are unaware of the situational difference in question, no information about such a difference is conveyed by any linguistic forms they use. The reason for saying this is simply the commonsense reason that if we are prevented by ignorance from drawing even a hazardous inference from $\underline{p}$ to $q$, or the probability of $q$, then $\mathfrak{p}$ can in no sense inform us that q. For example, for a strictly monogamous community unaware of the connexion between sexual intercourse and childbirth, it would be nonsense to identify the informational difference between their use of the word for a woman's offspring and the word for a woman's male consort after the birth of an offspring with that between English father and child. The condition of 'informationality' stipulates that it would be irrelevant for the linguist to establish that the one word applied only to fathers and the other only to their children; since the community ex hypothesi lacks the relevant concepts, whatever informational difference there might be uses of the two words, it could not be, either in whole or in part, that difference. Likewise, if in a given language there is only one mid back vowel, and in the north it is realized as [0], whereas in the south it is realized as [0] the correlation is 'informational' only if there is recognition of [o] as a 'northern' pronunciation and [J] as a 'southern' pronunciation. For without such recognition, the difference in pronunciation between e.g. [pot] and 
[pot] cannot in the relevant sense give information about the speakers, i.e. information about their provenance: it thus fails to qualify as a conventional phonic difference relevant to the analysis of the expressive function of speech in that community.

The condition of 'informationality', it should be noted, is not based upon any specific view of the proper way to define the 'meaning' of an expression, but simply upon the point that a language is an instrument of communication and the epistemological principle that what is not known cannot be communicated. 2.46 Subject to these two conditions, the phonologist's criterion of synonymity may be formulated as follows:

'Two phonetic types are realizations of synonymous expressions if they show no difference of usage and the phonetic difference between them is also that between other pairs of phonetic types which do show a difference of usage.'

This criterion goes no further than giving a sufficient condition for synonymous expressions, and this is adequate for purposes of phonological analysis, since the aim of the phonologist is here limited to discovering the phonological distinctions of I. To draw up a complete inventory of synonymous pairs for L lies outside his province qua phonologist. He is interested merely in such syhonymous pairs as have phonetic realizations sufficiently alike to raise the question of whether or not he is dealing with phonologically identical items. These are the cases in which it may be in doubt whether it is correct to account for the facts of communication by setting up one expression, or two expressions differing in form but not in meaning. 
2.47 Thus the essential feature of synonymity statements here is their role as providers of an alternative explanation of the fact that two phonetic types may have no discoverable difference of usage.

The role is a vital one because without this alternative the phonological analysis breaks down. If synonymity statements are eliminated, the case of phonetic types not differing in usage can be dealt with in only one of two ways; either (i) by assigning the same phonological form to both members of the pair, irrespective of other pairs showing the same phonetic difference, or (ii) by treating the pair in question as realizations of expressions differing in both form and meaning. But ( $i$ ) will lead to inconsistency in the phonological classification of the phonetic difference should there be other pairs where the same difference proves to be correlatable with a difference in usage, while accepting (ii) introduces the paradox of two expressions allegedly differing in both form and meaning but not differing in usage. To admit either possibility would be tantamount to admitting the inadequacy of the analytic procedures employed. 
3. Synonymy and grammatical analysis 
3.0 At the level of grammatical analysis, there are intimate connexions between the concept of synonymy and those of morpheme variant (or allomorph), grammatical ambiguity, and optional transformation.

3.1 The question of morpheme variants raises a prior general issue: to what extent is 'sameness of meaning' relevant at all to morphological analysis? As in the case of phonological analysis, two views stand in conflict. On the one side it is maintained that morphological analysis requires semantic information about the data under analysis, and that semantic considerations enter into the definition of morphological units. Hence such definitions of the morpheme as 'a linguistic form which bears no partial phonetic-semantic resemblance to any other form ' I, or 'any form... which cannot be divided into smaller meaningful parts' ${ }^{2}$, or, 'the smallest individually meaningful elements in the utterances of a language ${ }^{3}$. On the other side, it is maintained that criteria of form and distribution alone are adequate for morphological analysis, and so morphological units may be defined without reference to semantic considerations. Hence such assertions as 'distribution suffices to determine phonemes and morphemes and to state a grammar in terms of them ${ }^{4}$, or 'it would certainly not be impossible to determine roughly the morphemes of long enough printed texts without taking meaning into consideration ${ }^{5}$.

1. Bloomfield 1935 p. 161.

2. Bloch \& Trager $1942 \$ 4.2$.

3. Hockett 1958 14.1.

4. Harris $1954 \$ 5$.

5. Bazell 1954. 
Two questions concerning synonymy therefore arise: (i) is it possible to make good the claim, inherent in the procedures of nonsemantically based morphology, that morphological analysis does not require a concept of synonymy? (ii) supposing this claim to be false or irrelevant, what then is the function of a concept of synonymy in relation to morphological analysis?

3.11 The answer to the first of these questions depends on how the scope of morphological analysis is envisaged. To avoid involvement with the varying interpretations of the terms 'morpheme', 'morph', 'allomorph', etc., we may call the units with which morphology deals 'M-units', and distinguish between various views of the scope of morphological analysis by reference to different concepts of such a unit. If it is observed that in a language there are certain restrictions on the occurrence of phonemes or sequences of phonemes which cannot be brought within the scope of plausible phonological rules, and if it is desired to account for these restrictions in terms of units of a different order from the phoneme, such units (M-units) may be set up without appeal to meanings. 1 This assertion commits us to no assumption about the structure of the language in question, nor about the structure of language in general (apart from propositions to the effect that languages

1. No need arises to offer an argument in support of this proposition. Since the sequences governed by the restrictions are given as sequences of non-meaningful units (phonemes), the problem is no different from that of stating macro-unit combinatorial rules for any set of non-linguistic micro-unit sequences (e.g. the marks of a wallpaper pattern). Argument, on the other hand, would be required in oupport of the opposite view, namely the assumption that semantic information is necessary in order to do this. 
manifest, or may manifest, nonphonological distributional constraints). Quite a different matter is whether the M-units set up to account for nonphonological patterns of distribution have determinate meanings, and an answer, whether affirmative or negative, to this question ${ }^{2}$ would commit us to a proposition about linguistic structure. A different question again would be whether, supposing the answer to the latter question to be 'yes', M-units are better determined by means of semantic or non-semantic criteria. A view about this has no implications concerning linguistic structure, however, for the question belongs to the empirical methodology of morphological analysis.

3.111 Clearly, the decision to set up M-units in a particular case will depend on the usefulness of so doing in order to account for certain facts of (non-phonologically determined) distribution. But these include facts of varying generality and different kinds of importance. It is therefore slightly beside the point to cite, as does Zellig Harri. ${ }^{1}$, cases such as English persist and person, in an attempt to show the superiority of non-semantically based morphology. Harris argues that 'if the morphemic composition of a word is not easily determined, we cannot decide the matter by seeing what are the component meanings of the word and assigning one morpheme to each. Do persist, person contain one morpheme each or two? In terms of meaning, it would be difficult to decide, and the decision would not necessarily fit

2. Strictly speaking, there are at least two questions here: (i) whether M-units have meanings, (ii) whether M-units as such have meanings.

1. Harris $1954 \oint 4$. 
into any resulting structure. In terms of distribution we have consist, resist, pertain, contain, retain, and the like (related in phonemic composition and in sentence environment), but no such set for person; hence we take persist as two morphemes, person as one'. But all this example shows is that there is a non-semantic criterion available which gives a certain solution. There is also, however, a semantic criterion available which gives a different solution (i.e. persist and person as single M-units on the ground that no cut yields independently meaningful segments). What needed demonstration to prove Harṛis's point was not the availability of some non-semantic (distributional) criterion, but either (a) the existence of a 'correct' answer to which the distributional criterion leads directly, but the semantic criterion dubiously, if at all, or (b) that the solution given by the distributional criterion is useful, whereas that given by the semantic criterion is not, or less so. But, as regards (a) it would clearly be question-begging to assume that the analysis per-sist is correct and per-son incorrect in order to show the improbability of producing a correct analysis by semantic criteria. As regards (b), no attempt at all is made to assess the utility of the preferred analysis, which in fact turns out to be of a somewhat low order. We can thereby, it is true, subsume certain morphological facts about English verbs under a general rule (e.g. 'Inglish verbs admit the structure $A B$ but not $B A$ where $A=$ 'per-, re-, con-...' and $B=$ '-sist, -tain....'); but the same facts can be stated hardly less economically on the supposition that the forms are monomorphemic 
('The class of English verbs includes persist, resist, consist, pertain, retain, contain .... but excludes *sistper, *sistre, "sistcon, *tainper, *tainre, *taincon ..." $)^{2}$.

Moreover, the facts in question are relatively unimportant in the sense that an infringement of the rule merely produces a non-Bnglish lexical item in an otherwise grammatical snglish arrangement (e.g. *The defenders did not sistre for The defenders did not resist), which is tantamount to saying that no basic grammatical distinction of English would be obliterated if the language admitted both the permitted and the excluded sequences. Admission of the excluded sequence would merely require that the lexicon of Inglish be expanded to accommodate such synonym pairs as resist/sistre, contain/taincon, etc. 3

2. There is no comparison here with the situation which would arise if a criterion gave he eats, they eat etc. as monomorphemic, and a rule consequently had to exclude specifically by listing whe eat, *they eats etc.

3. Furthermore, the apparent determinacy of the non-semantic solution in Harris's example is to some extent illusory. It is doubtless easy to find analogies which will support splitting persist into per-sist. Nonetheless, some can be found, if one looks hard enough, to support splitting person into per-son (e.g. parson, mason, pervert, Persian). What remains unanswered, if one relies on non-semantic criteria, is the question how many supporting analogies have to be found, and how closely they have to conform to the distribution of the form under analysis. Any investigation of such questions is likely to reveal a continuous scale of 'phonetic-distributional Iikeness', which leaves the investigator - not the criterion to decide when there is a 'sufficient likeness' to support one analysis rather than another. 
3.112 On the other hand, if morphology is envisaged as extending to include what may be called 'M-relations' (e.g. 'plural of', 'past tense of'), then it becomes questionable whether non-semantic criteria can produce an adequate analysis. It would seem that the most powerful theory of $M-$ relations achievable on the basis of non-semantic criteria is one which would be incapable of explaining e.g. what is neant by describing went as the past tense of go.

The units between which M-relations hold are items which traditional grammar normally treats as different morphological forms of the same 'word', e.g. look and looked, bonus and bonum, je and moi, and it is relevant to observe that the metalanguage of traditional grammar acknowledges the distinction between M-relations and mere grammatical classification: e.g. one says that looked is the past tense of look, or that bonum is the neuter of bonus, or that moi is the strong form (disjunctive form) of je, whereas of looked and see, or of bonum and clarus, or of moi and $\underline{\text { il }}$, one does not say that one is any kind of form of the other - instead, the similarities or differences in their grammatical behaviour are described by simple classification (e.g. by categorizing them as 'verb', 'past', 'adjective', 'neuter' etc.) ${ }^{\mathrm{I}}$.

1. The term 'grammatical synonym' has been proposed by Winter for sets like English walked, went and came, and 'may be applied equally well to entire constructions and to fractions of constructions, whenever these can be isolated by strictly formal procedures' (Winter 1964 pp. 14-15). By this is meant that the forms 'are synonymous in respects other than lexical meaning'. This proposal, however, stands in need of considerable clarification, since according to Winter 'the basic characteristics of a set of synonyms are that they have a comparable range of dj.stribution (or ne:zining) - they are synonymous only to the extent that they do just that -'. It is not clear exactly how to take the 'or' here in 'distribution (or meaning)', and this is one reason why it is not clear whether e.g. pairs like boy and girl would 
But it is a non-trivial fact about natural languages that M-relations may obtain between items not identifiable by criteria of formal resemblance, and this fact precludes one possible basis for an account of M-relations which would exclude semantic considerations. This is perhaps best illustrated by means of a hypothetical example.

Suppose that ziff is an English verb occurring in such sentences as I usually prefer to ziff it, whey turn out better if you ziff them, etc., and the question arises how we may determine whether or not ziffed is the past tense of ziff. The claim that we may resolve this question by using exclusively non-semantic criteria implies of course that we do not need to take into consideration the distribution of these items in semantically acceptable contexts, but simply their distribution in the grammatical sentences of English. Now if ziffed is in fact the past tense of ziff, ziffed will occur in a set of environments in which it is substitutable salva grammaticalitate for such forms as spliced, crossed, missed etc., which are not presumably correctly describable as 'past tense forms of ziff.' That is to say, granted distributional definitions of the notions 'past tense form' and 'present tense form' such that ziff, splice etc. count as presents and ziffed, spliced etc. as pasts, we are still left without a criterion to effect unique M-relation pairings between members of the two classes. Therefore, to be the past tense of ziff cannot be simply to be a member of a certain class of forms

count as 'grammatical synonyms'. Another reason is that unless some criterion is proposed for determining exactly what is included in 'lexical meaning', it is difficult to decide whether forms 'are synonymous in respects other than lexical meaning'. 
interchangeable salva grammaticalitate. But all that apparently remains to distingui.sh (non-semantically) ziffed from spliced, crossed, missed, etc. in a way that clearly links ziff with ziffed and excludes the pairings ziff - spliced etc. is the formal (i.e. phonological) constitution of ziffed. If, however, this is the sole differentia which singles out ziffed as that member of the class of past tense forms which is the past tense of ziff, it becomes apparent that the notion 'past tense of' reduces to the conjunction of a distributional classification (i.e. 'past tense form') and a phonological classification (i.e. $1 / z i f /+\ldots 1)$.

The example may be taken as illustrating a general strategy for reducing any M-relation to concepts which remain within the limits of non-semantically based morphology. But against anyone who is tempted to defend this reduction by maintaining that an M-relation is just the conjunction of a distributional and a phonological classification, two points must be made. First, that if so, then there are indefinitely many M-relations, most of which no-one would dream of calling a grammatical relation at all (e.g. that between an English noun and English adjective with a phonologically common segment: dent, dental; gent, gentle; roof, rueful; etc.) $)^{2}$ Second, that in cases of the relationship between forms

2. If it is counterargued that reducing M-relations to distributionalcum-phonological classifications does not commit us to the view that all such classifications define M-relations, the question then arises how we know which classifications do and which do not, and to this there seems to be no answer which can be arrived at by invoking solely non-semantic criteria. The reductionist cannot both have his bun and eat (part of) it. 
where the phonological resemblance involved is nil (as e.g. in saying that went is the past tense of go) there is no basis for identifying the invariant which is implied in the concept of an M-relation. For any useful concept of an M-relation must be essentially that of a two-place predicate $\underline{M}$ such that to assert $\underline{M}(\underline{a b})$ implies the existence of some linguistic entity realized in common by $\underline{a}$ and by $\underline{b}$. Pursuing the analysis further, it is evident that statements to the effect that $\underline{b}$ is the past tense of a have the underlying logical form:

$$
\exists \times \quad\left(\underline{M}_{1}(\underline{a x}) \cdot M_{2}(\underline{b} x)\right)
$$

- that is to say, we assert that there is an entity $\underline{x}$ (in this case a verb) such that $\underline{a}$ is its infinitive form and $\underline{b}$ its past tense form. But if there were to be cases where phonological resemblance or lack of it between $\underline{a}$ and $\underline{b}$ just did not count, then the M-relation would amount simply (by the reductionist thesis) to a distributional classification. This means that the past tense of a in such a case must be reckoned to be any item which falls into the distributional class 'past tense form', and this consequence is clearly intolerable. W.g. if we suppose that ziff may have a past tense form phonologically unrelated to ziff, we are left with no plausible method of identifying the form in question among the members of the class of English past tense forms, whether or not that class includes ziffed.

Nor can this reductionist dilemma be avoided by treating such instances as deviations from a standard case in which both phonological. and distributional criteria are involved, as e.g. to say that went is to go 
as looked is to look $^{3}$, except that the phonological resemblance in the former case is nil. For if appeal to meanings is dispensed with, then went can no more be established as the past tense of go than as the past tense of any other verb lacking a phonologically 'regular' past tense form. (If there happens to be only one 'irregular' pair in the system, a simple process of elimination will ensure the correct pairing; but good luck should not be confused with adequacy of the criterion employed). We cannot in any sense 'suspend' the requirement of phonological resemblance in order to link went with go, as we might e.g. suspend a colourbar rule in order to let black Mrs. Smith accompany her white husband. Waiving a general rule in favour of a particular relationship assumes that the relationship is established - or in principle establishable in advance of the rule. But this will not do where the purpose of the rule is precisely to establish such relationships.

Parallel arguments can be adduced for any M-relation. It must be concluded that a theory of M-relations which explicates then solely in terms of phonological-cum-distributional classification is ill suited to the purposes of linguistic analysis, since it embraces on the one hand relations of no conceivable linguistic interest, while sometimes excluding on the other hand relations between items having functions exactly analogous to those between which M-relations hold. There is simply

3. The proportion is inexact in that went does not function as a past participle, as does looked; but this may be ignored for purposes of the example. 
no point in having such a theory of M-relations to account for how natural languages work. For all it can do in the way of accounting for non-phonologically conditioned distribution patterns can already be done without reference to M-relations at all ${ }^{4}$. Whereas if we seek to account for the morphological phenomena of 'variable words' in general, we shall need a more powerful theory which covers both cases where there is and cases where there is not a parallelism between form and function. 3.113 A more radical reductionist thesis, avoiding appeal to phonological resemblance altogether, is embodied in the proposal that the morpheme, as distinct from the morph, be regarded as a factor of distributional proportion. Iyons argues in favour of this proposal as follows:

'To say that worse is composed of two morphemes, one of which it shares with bad (and worst) and the other of which it shares with taller, bigger, nicer, etc., is equivalent to saying that worse differs from taller, bigger, nicer, etc. in grammatical function (that is, in its distribution throughout the sentences of English) in the way that bad differs from tall, big, nice, etc. (and worst from tallest, etc.). This is commonly expressed as a

4. E.g. by recognizing as M-units such forms as he, she, they, and also eat, eats, find, finds, and formulating a selectional rule which forbids the combinations *he eat, *they eats, etc., we can account for restrictions of great generality in English. But in order to do this we do not need to say e.g. that they is the plural of he, she, or that eats is the third person singular of eat; introducing M-relations adds nothing to the precision or generality of the selectional rule. 
proportion of grammatical, or distributional, equivalence...

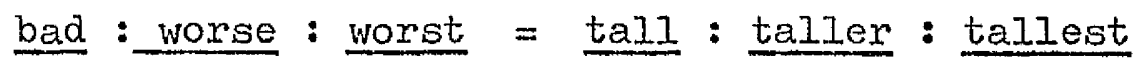
This proportion expresses the fact that, for example, worse, and taller (as well as bigger, nicer, etc.) are grammatically alike in that they are the comparative forms of the adjective they can occur in such sentences as John is worse (taller, etc.) than Michael, It is getting worse (taller, etc.) all the time. Worse and taller (as well as bigger, nicer, etc.) differ from one another, however, in that they cannot occur in exactly the same set of sentences - for instance, as traditional grammarians would say, they cannot 'qualify' exactly the same set of nouns. In so far as the class of nouns which can be qualified by a particular adjective is grammatically determined ... this feature of their distribution is accounted for by postulating a particular morpheme as a component of one adjective and another morpheme as a component of another adjective which 'qualifies' a different class of nouns ${ }^{1}$. Interpreted as a theory of M-relations, this means that a statement to the effect that the comparative of bad is worse (or 'bad + -ex $\rightarrow$ worse') reduces to a distributional proportion bad : worse $=\underline{\text { tall }}:$ ta.lier etc. ${ }^{2}$

1. Lyons $1968 \$ 5.3 .3$.

2. i.e. the invariant implied in saying that $a$ is the comparative of $\underline{b}$ is identified by means of a distributional factor common to $a$ and $\underline{b}$ : the $\underline{x}$ of which a and $\underline{b}$ are both forms is assumed to be characterized by this distributional property. 
The first point which calls for comment about this proposal is that, conspicuously, one of the environments in which tall occurs is the environment '...-er', and it is just the fact that bad does not occur in this environment which gives rise to the need for factorization. Thus it would seem that the proportion bad : worse $=\underline{\text { tall: }}$ taller cannot be an identical proportion (in spite of the sign ' $={ }^{\prime}$ ), for it is possible to cite a respect in which the distribution of bad is not to that of worse as the distribution of tall is to that of taller, namely in the respect that in the one case there is and in the other case there is not an environment which, by insertion of the first form, yields the second form (which is a distributional feature of very many Inglish positivecomparative pairs).

This observation prompts the further question: what, then, exactly is the statement of proportion intended to assert? Is the exception cited above the sole exception to an otherwise identical proportion? If we exclude by stipulation from the distribution that counts just any contexts which occur as morphs in the proportion, we presumably arrive at a statement of the following kind: 'Excluding the environment '...-er', the distribution of $\underline{\mathrm{bad}}$ is to that of worse as that of tall is to that of taller, etc.' This is a statement which appears to make an empirical claim of a quite specific kind, yet when we come to consider how. we might test it, it emerges that it is by no means clear precisely what the claim is.

According to (part of) Lyons's explanation, worse is alleged to differ distributionally from taller in the same way as bad differs from 
tall. But what, in turn, does that mean? Is to say that A differs distributionally from $B$ in the same way as does $C$ from $D$ to say that any environment in which $A$ occurs but not $B$ is also an environment in which $C$ occurs but not D? Or is it to say that those environments in which $A$ occurs but not $\mathrm{B}$ are all and only the environments in which $\mathrm{C}$ occurs but not D? Or is it to say merely that a common feature can be found which characterises those environments in which $A$ occurs but not $B$ and also those in which $\mathrm{C}$ occurs but not $\mathrm{D}$ ? Or is to to say, even more merely, that a condition $q$ can be established such that if $q$ then either $A$ but not $B$ or $C$ but not $D$ ? lyyons introduces the notion of 'similarity of distributional differences' by way of explanation: but until this notion itself is given some substantive content we cannot tell what it is that statements of distributional proportion assert.

However, it is not necessary to wait upon this enlightenment in order to see that, as a theory of M-relations, any attempted reduction to distributional factors has a fundamental flaw. In a proportion of the form 'A : $\underline{x}=C: D$ ' where, for the sake of argument, the distributional relation of $\mathrm{C}$ to $\mathrm{D}$ is that of distributional identity, any candidate for $x$ must be distributionally identical with $A$. If there is no form in the language with such a distribution, no $\underline{x}$ will be forthcoming. But if there is more than one such form, more than one $x$ will be forthcoming. The same holds, mutatis mutandis, for cases of inclusive distribution, overlapping distribution and complementary distribution - in short, for whatever condition is imposed by the distributional proportion. It follows that the equation of M-relations with distributional relations 
yields absurd consequences, e.g. that any English form which happens to have a distribution identical with that of bad must (irrespective of form and meaning) have worse as its comparative. That there may be no such form in English does not affect the argument. The existence or nonexistence of a form distributionally identical with bad is an empirical fact, and the theory provides no guarantee against contradiction by such facts. There is an assumption, in other words, that it is a property of natural languages that any two forms $\underline{a}$ and $\underline{b}$ uniquely paixed by an M-relation are terms of a distributional relation which obtains for at least one other pair of forms, and that either a or $\underline{b}$ is distributionally unique. But this does not follow from any postulate of linguistic analysis; nor is any general reason apparent why it should be held to be true - except, circularly, to validate the theory of M-relations under discussion.

3.114 It might perhaps be urged that at least a semantically based theory of M-relations is no better off, since it leads to the parallel conclusion that any English form which happens to be synonymous with bad must have worse as its comparative. But no-one is obliged to argue that M-relations are determinable by semantic criteria alone: the point at issue is whether semantic criteria can be excluded. If anyone did maintain that an English adjective synonymous with bad must ipso facto have worse as its comparative, this would not be totally absurd, and well known facts of historical linguistics might be adduced to support it. A word a may 'take over' or 'assimilate' formally disparate elements from another word $\underline{b}$ if the semantic contrast between $\underline{a}$ and $\underline{b}$ lapses: 
e.g. the Spanish verb ir 'to go' (from Latin ire) has acquired present tense forms which originally belonged to Latin vadere ( $\mathrm{Sp}$. voy, vas, etc.), and this replacement presupposes a transitional state in which eo and vado were both 'first person present indicative' of ire. 3.12 Reverting now to the question of M-units, it is clear that if M-units may be set up to account for non-phonologically conditioned distributional patterns without recourse to meanings, then since such an enterprise needs no analysis of meanings, a fortiori it requires no concept of synonymy. This, however, leaves open the question of whether the enterprise could be carried out with M-units established on a semantic basis. It is not difficult to imagine a language structured in such a way that it would be quite impractical to attempt to state all or even most of the non-phonological sequence restrictions in terms of semantically determined units. (Such a language would have a 'third articulation', two of its three articulations being non-meaningful). A semantically based morphology (of which the general feasibility is not denied by 'distributionalists ${ }^{\prime}$ ) is intimately related to the assumption of dual articulation as a universal property of natural languages. But it does not follow from this assumption, granted its correctness, that

1. Harris $1951 \oint 12.41 \mathrm{n} .67$ : '..there is in general a close correspondence between the morpheme division we might establish on a meaning basis and that which results from our distributional criteria. This is so because in general morphemes which differ in meaning will also differ in their environments, if we take sufficiently long environments and enough of them.' But this is not in fact a reason unless the point is tacitly conceded that natural languages have no important classes of non-meaningful morphemes. 
units established on a semantic basis are readily available for stating every grammatical fact. In support of this, one need look no further than the frequent discrepancies between proposed semantic definitions in morphology and some of the items occurring in natural languages to which the proposer is prepared (or not prepared) to apply the term. Such discrepancies usually arise from attempts either to 'stretch' the morpheme to cover units which it is morphologically useful to have included, but which happen not to be readily definable on a semantic basis, or else to 'shrink' it, so as to exclude troublesome items which appear, on semantic grounds, to have some claims to be included. Examples of 'morpheme-stretching' and 'morpheme-shrinkage' can be found in the presentations of semantically based morphology by Bloomfield, Bloch \& Trager, and Hockett. Others could be cited, but the examples examined below are typical of the phenomenon. The relevant arguments clarify various points which have a bearing on questions of synonymy in relation to grammatical analysis.

3.121 The three definitions of the morpheme already cited in 3.1 (i) 'a linguistic form which bears no partial phonetic-semantic resemblance to any other form', (ii) 'any form... which cannot be divided into smaller meaningful parts', and (iii) 'the smallest individually meaningful elements in the utterances of a language' - are by no means equivalent, but share a common appeal to semantic considerations. Definitions (ii) and (iii) require us to be able to decide simply whether or not certain items are meaningful, whereas (i) imposes the further requirement of determining a likeness of meaning between items 
(assuming that to speak of a semantic resemblance is not merely to speak of a resemblance which consists simply in being meaningful). However, the envisaged applications of ( $i$ ) and ( $i i i$ ) also involve this further requirement, as will become apparent below. 3.122 Definition (i) - Bloomfield's - assumes that we are dealing with meaningful expressions to which a determinate phonological form is assignable, and proposes a bipartition of this class of expressions into two disjoint classes (called 'simple' and 'complex'), using 'phoneticsemantic resemblance' as the criterion:

'... some linguistic forms bear partial phonetic-semantic resemblances to other forms; examples are, John ran, John fell, Bill ran, Bill fell; Johnny, Billy; playing, dancing; blackberry, cranberry; strawberry, strawflower. A linguistic form which bears a partial phonetic-semantic resemblance to some other form is a complex form. The common part of any (two or more) complex forms is a linguistic form; it is a constituent (or component) of these complex forms ${ }^{l}$.

The constituents in the examples quoted are said to be: John, ran, Bill, fell, play, dance, black, berry, straw, flower, cran-, -y, and-ing. The requirements of morphological analysis as envisaged in the foregoing statement appear to be three. 1. The linguist must be able to determine with respect to any given linguistic form whether that form is simple or complex. 2. In order to satisfy himself that it is complex 
he must be able, in the simplest type of case, to analyse it as a bipartite form $\underline{a b}$, and to show that either $\underline{a}$ or $\underline{b}$ occurs elsewhere in the language as a linguistic form or part of a linguistic form. 3. In order to satisfy himself that it is simple, he must be able to show that the form does not yield to analysis in such a way that at least one part occurs elsewhere in the language as a linguistic form or part of a linguistic form.

An assumption underlying these requirements is that a linguistic form is determinate with respect to meaning, and that this meaning is ascertainably constant for all instances of the linguistic form. 3.123 A somewhat different assumption is made in Bloch and Trager's presentation of definition (ii):

\section{'As we examine the}

recorded uttexances of an informant or a speech community, we note that the same or similar forms recur again and again with the same or similar meanings. Thus, the utterances of an English-speaking informant wil1 contain many instances of such forms as yes, person, I think so, out of town, each time with about the same meaning's, and also such different but phonemically related forms as play, plays, played, playing, or ride, rides, rode, ridden, riding, or man, manly, mannish, or conceive, perceive, conception, perception, perceptive, with different but related meanings.

On the basis of such recurrence, we analyze the utterances into fractions of various lengths, each with a more or less constant meaning. Any fraction that can be spoken alone with meaning in normal speech is a 
FREE FORM; a fraction that never appears by itself with meaning is a BOUND FORM. All the examples in the preceding paragraph are free forms; per-, con-, -ing, -1y, -ish, -ceive, tion are bound forms ${ }^{l}$.

A feature of this account is its supposition that morphological analysis has a certain semantic discovery structure. Some semantic information (about sameness or similarity or relatedness of meanings of expressions) is assumed to be available to empirical observation, and on the basis of this information the linguist constructs hypotheses about meanings not 'observable', namely those of the constituents of the expressions whose meanings are already known. The morpheme is the unit reached when factorial analysis of known or hypothesized items with their meanings can proceed no further.

3.124 A similar concept of the morpheme as the ultimate unit arrived at by a process of factorial analysis lies behind definition (iii) Hockett's. The twin tests he suggests for the identification of any segment of a sentence as a morpheme are these: (I) that the segment recur in other utterances 'with approximately the same meaning', and (II) that it should not be the case that the recurring segment can 'be broken into smaller pieces, each of which recurs with approximately the same meaning, in such a way that the meaning of the whole form is related to the meanings of the smaller pieces' ${ }^{I}$. By these criteria, it is claimed, Bnglish older consists of two morphemes, one recurring in e.g. oldster, oldest and the other in e.g. younger, finer, while sister is monomorphemic, since neither sist-er nor any other division gives units which can

1. Bloch \& Trager $1942 \oint 4.2$.

1. Hockett $1958 \$ 14.1$. 
plausibly be identified as having 'reasonable similarity of meaning' with phonemically comparable units in other utterances.

3.125 The definitions discussed above and their application to the morphological analysis of natural languages give rise to various important questions concerning identification of meanings. One which may profitabiy be discussed here concerns so-called 'unique' morphemes, since what is at issue in this case is the legitimacy of applying what purports to be a meaning-based definition of the morpheme in such a way as to yield criteria for identification of meanings, which will, in turn, enable certain otherwise excluded items to be classified as morphemes.

Unique morphemes make their appearance in morphological analysis as presented both by Bloomfield and by Hockett, and the justification given for their recognition is as follows.

1. (Bloomfield) '... having heard the form cranberry, we soon recognize the component berry in other forms, such as blackberry, and may even hear it spoken alone, but with the other component of cranberry we shall have no such luck. Not only do we wait in vain to hear an isolated * cran, but, listen as we may, we never hear this element outside the one combination cranberxy, and we cannot elicit from the speakers any other form which will contain this element cran-. As a practical matter, observing languages in the field, we soon learn that it is unwise to try to elicit such forms: our questions confuse the speakers, and they may get rid of us by some false admission, such as, "Oh, yes, I guess cran means red." If we avoid this pitfall, we shall come to the conclusion that the element cran- occurs only in the combination cranberry. 
However, since it has a constant phonetic form, and since its meaning is constant, in so far as a cranberry is a definite kind of berry, different from all other kinds, we say that cran-, too, is a linguistic form' 1 .

2. (Hockett). 'Occasionally, after we have extracted all the morphemes from some utterances by successive applications of Tests $I$ and $I I^{2}$, we seem to have something left over. Consider, for example,

\section{${ }^{2}$ pléase páss the ${ }^{3}$ cránbèrries ${ }^{\top} \downarrow$}

The last word of this sentence can obviously be broken into cranberry and a morpheme / $\mathrm{z} /$ meaning "plural". Cranberry, in turn, seems clearly to contain an element/bèrij/ which recurs in strawberry, raspberxy, gooseberry, blackberry, blueberry, and so on. But how about cran-? We look in vain for any recurrence of cran- with anything like the meaning it has in cranberry.

A strict adherence to Tests I and II would therefore force us to take cranberry as a single morpheme. Yet this is obviously undesirable. In the first place, the identity of the second part of cranberry is hardly subject to doubt. In the second place, cran- clearly carries a meaning, even if the element occurs nowhere save in this one combination. Cranberries are different from strawberries, raspberries, gooseberries, and so on; the meaning of cran- is therefore whatever it is which differentiates cranberries from those other kinds of berries. It might be

1. Bloomfield 1935 p. 160 .

2. Cf. 3.124 . 
hard to describe this meaning, but it is easily demonstrated in a fruit market.

What we do under such circumstances is to recognize the element in question as a morpheme of a rather special kind - a unique morpheme. The recognition of such special morphemes does not require any modification of our definition, but only calls for a slight change in the way we apply Tests I and II 3 .

The question of unique morphemes is of interest because it points up a conflict between the enterprise of giving a semantically based definition of the morpheme and the desideratum that morphological analysis should be an analysis without residue. For if it proves possible to analyse an expression into ab such that a clearly occurs elsewhere with the same meaning, but $\underline{b}$ does not appear to, then we have the choice either of saying that there is a residue $\underline{b}$ whose status is unaccounted for, or else of declaring $\underline{b}$ a morpheme. What is dubious is whether we can opt for this latter alternative while maintaining unchanged or unsupplemented our original definition of the morpheme. It is the attempt to do this which leads Bloomfield and Hockett to deploy some very bad argumentis.

Bloomfield contends that the meaning of cran- is constant 'in so far as a cranberry is a definite kind of berry', thus committing the double error of nonsense and non sequitur. What makes sense (and is true) is not that a cranberry is a kind of berry but that a cranberry is a kind

3. Hockett $1958 \oint 14.2$. 
of berry. But it does not follow from that, nor from the fact that cranberry and berry are words for cranberries and berries respectively, that the meaning of cran- is constant. For it does not follow, quod erat demonstrandum, that cran- has a meaning at all (unless we define 'having a meaning' in such a way that being a unique element of the kind described is a sufficient condition for having a meaning - which would be clearly circular in the present instance).

Hockett, on the other hand, claims that since cranberries are different from strawberries, raspberries, gooseberries, etc., the meaning of cran- is therefore 'whatever it is which differentiates cranberries from these other kinds of berries'. Here, Bloomfield's non sequitur is compounded by a further non sequitur, namely the identification of the meaning of cran- with the whole set of differentiae of cranberries as a subclass of berries ${ }^{4}$. For there is no reason, granted that cran- has a meaning, why that meaning should be equated with all rather than any of the differentiae of the subclass of cranberries, nor, further, why we are obliged to equate it with a differentia of cranberries at all. (Cran- might be, as Bloomfield's hypothetical informant suggested, an otherwise unattested morpheme meaning e.g. 'red'.) The method of assigning meanings by simple 'semantic subtraction' is open to the objection levelled by Bazell ${ }^{5}$ against 2.S. Harris's proposal that 'the

4. It is not entirely clear whether Hockett's thesis is that, or whether he holds the meaning of cran-to be whatever differentiates cranberries from other berries having names ending in -berry. He seems at times to be advancing the former and at times the latter view, without apparently realizing that this is to ascribe two different meaning's to cran-.

5. Bazell 1954 p. 330. 
meaning of blue in blueberry might be said to be the meaning of blueberry minus the meaning of berry': 'one could give any element whatever a meaning in this sense: e.g. the meaning of $\underline{b}$ in beat would be the difference between the meaning of beat and the meaning of eat." Should it be replied that this reductio ad absurdum is unfair, since there is no presumption that the meanings of beat and eat are related, it would then be legitimate to point out that the blueberry example trades on the opposite presumption, namely that the meanings of blueberry and berry indeed are related. Applied without any such presumption, the method of 'semantic subtraction' yields trivial results: e.g. the meaning of blue in blueberry is the meaning of blueberry minus the meaning of berry, and, on the other hand, the meaning of berry in blueberry is the meaning of blueberry minus the meaning of blue.

Tven if the semantic identity between berry standing alone and the second elements in blueberry and cranberry be conceded, the operation of 'semantic subtraction' is on no sounder theoretical footing. For, in general, for any bi-partite meaningful expression $\underline{a b}$ where a but not $\underline{b}$ recurs with the same meaning elsewhere, it does not follow that $\underline{b}$ has a meaning. The fact that the language may have comparable expressions of the form ac, ad, etc., where both elements recur with the same meanings elsewhere, affords no demonstration of the meaningfulness of $\underline{b}$ in $\underline{a b}$, since it does not follow from any postulate of linguistic analysis that related expressions must have identical semantic structures. In assigning a meaning to $\underline{b}$, the linguist in fact yields to the same temptation as Bloomfield's informant - to be rid of the embarrassing question 'What does 
it mean?'.

3.126 There are other awkward cases which semantically based morphology seeks either to accommodate or to dismiss by disregarding its own proclaimed definition of the morpheme.

One such case is examplified by series of forms such as:

1. slime, slush, slop, slobber, slip, slide, slurp, slick.

2. flash, flare, flame, flicker, flimmer.

- where the members do seem to bear a phonetic-semantic resemblance to one another, which might be stated in the instances quoted as

and

$$
\begin{aligned}
& {[\mathrm{sl}-]=\text { 'smoothly set' }} \\
& {[\mathrm{fl-}]=\text { 'moving light'. }}
\end{aligned}
$$

Bloomfield recognizes such elements as 'root-forming morphemes'I. He does not, however, isolate similarly an initial element [n-] = 'negation' in such a series as:

3. no, not, none, nor, never, neither. ${ }^{2}$

But in terms of the definition of the morpheme as a linguistic form which bears no partial phonetic-semantic resemblance to any other, series 1, 2 and 3 stand or fall together.

Nida, on the other hand, refuses to recognize the elements [sl-] and [fl-] of series 1 and 2 as morphemes, because in spite of the 'partial phonetic-semantic resemblances', the elements in question 'do not occur with free forms or with forms which occur in other combinations ${ }^{3}$. Two observations are in order concerning this objection. First, it is in fact not a valid objection to such cases as none, nor, never,

$\begin{array}{lll}\text { 1. Bloomfield } 1935 & \text { p. } 245 . \\ \text { 2. Bloomfield } 1935 & \text { p. } 244 . \\ \text { 3. Nida } 1949 & \text { p. } 61 .\end{array}$


neither (where one, ever, either presumably qualify as free forms) nor even to cases such as flash (in view of bash, clash, crash, dash, where $[-a[]=$ 'violent novement'). Second, valid or not, the objection appeals to a principle which has no justification in terms of the given definition of the morpheme, i.e. the principle that elements not occurring in combination with free forms or with forms occurring in other combinations are not morphemes.

3.127 A further type of 'awkward case' is examplified by such series as: 1. conceive, deceive, perceive, receive.

2. attend, contend, distend, pretend.

3. adduce, conduce, deduce, induce, produce, reduce.

- where the usual view taken by advocates of semantically based morphology is to regard elements such as con-, de-, re-, -ceive, tend, -duce as morphemes, in spite of the difficulty of determining a semantic similarity. Bloomfield conceded that 'it seems impossible ... to set up any consistent meaning' for such elements ${ }^{1}$; but does not tell us why in that case they should be regarded as morphemes. Nida reasons as follows: "The prefix con- occurs only in combinations, e.g. conceive, consume, contain, condense, but the form dense occurs in isolation. This provides justification for considering con- a morpheme. Added evidence is available in the fact that the stem forms occur in other combinations, e.g. perceive, resume, detain $^{2}$. All that is missing here is the explanation of why anyone should regard con- as a morpheme for that reason. For no attempt is made

1. Bloomfield 1935 p. 154 .

2. Nida 1949 p. 59. 
to find the semantic element of a presumed phonetic-semantic resemblance theoretically required for establishing morpheme status.

3.128 Unsatisfactory analyses of the kind discussed in the preceding sections might lead one to question whether a definition of the morpheme based on 'phonetic-semantic resemblance' can be viable, since its champions are evidently so hard put to it to give an account of awkward cases. It is possible, on the other hand, that the definition merits vindication at the expense of its champions. One might come to the conclusion that there is nothing wrong with the definition, but a great deal wrong with the way its proponents pay it lip service in theory but deny it in practice. Perhaps all that is needed to set matters right is a consistent application of the definition in various types of case.

Some of the arguments advanced by 'distributionalists' may be met in this way. For example, the case is cited of 'empty' morphs such as the to in English I tried to swim, which is simply present by requirement of Inglish syntax, and thus held to be a counterexample to the thesis that morphemes are minimal meaningful units. The answer to this - if the charge of meaninglessness is conceded - is to withdraw the proposition that to is a morpheme ${ }^{I}$. For what is objectionable is not the intolerable consequences of denying so-called 'empty' morphs morphemic status, but the inconsistency involved in swelling the morpheme inventory of a

1. Weinreich $1963 \$ 1.2$ meets the objection in a different way by contending that 'empty' morphs are 'an artifact of an Item-andArrangement grammar: in an IP grammar, they are not "empty", but are the segmental markers of a transformation process'. It might still be objected that this is to fill the 'emptiness' with quite a different concept of 'meaning'. 
language by the addition of admittedly meaningless members.

Quite a different objection, on the other hand, would be the objection that a definition of the morpheme in terms of 'phonetic-semantic resemblance' is incapable of consistent application. The chief ground on which this objection might be raised is that since neither 'phonetic resemblance' nor 'semantic resemblance' are defined in any very precise way, it seems impossible to decide, except ad hoc in different cases, what constitutes a phonetic-semantic resemblance. Those who try to maintain a semantic justification for the morpheme status of elements like to sometimes lay themselves open to this objection. Gleason, for example, concedes that 'it is impossible to find a specific factor in the situation which can be considered as the "meaning" of to. Nevertheless, to does have a function, since without it "I want go means nothing'? But, of course, in. that sense the $\underline{w}$ of want also has a function, and moreover can be found with the same form and function in many other Inglish sentences (I want treacle, I wishyou'd stay etc.)

As regards the element of phonetic resemblance in a 'phoneticsemantic resemblance', the objection raises a point of some significance in connexion with synonymy. It would presumably be possible for the Bloomfieldian to meet the objection by falling back, if pressed, on a notion of phonetic resemblance which no-one could reasonably question, i.e. phonemic identity. Now a grammar constructed on the basis of this interpretation of 'phonetic-semantic resemblance' would have no room for 
synonymity statements, i.e. there would be no cases in which the determination of M-units depended on deciding whether two phonologically different items were alike in meaning. But a further and related property of such a grammar would be that the difference between e.g. the noun forms knife and knive (as in knives) would be exactly on a par with the difference between, say dock and dog, these four forms counting as four different M-units.

In other words, without appeal to the notion of synonymy in a grammar based on 'phonetic-semantic resemblance', the distinction between morph and morpheme collapses. Synonymity statements are required in Bloomfieldian grammar precisely to support the contention that a given morpheme may appear in various forms ${ }^{3}$, a requirement which makes Bloomfield's theoretical rejection of synonymity (1.3) all the more paradoxical. 3.13 The concept of synonymy plays a somewhat different role in semantically based morphology if the morpheme is defined not in terms of 'phonetic-semantic resemblance', but as a unit determined conjointly by semantic and distributional criteria, as e.g. in the account of morphological analysis given by Gleason ${ }^{1}$. Here the function of the concept is precisely to allow morphological analysis to proceed without any reliance at all on phonetic or phonological resemblance between morphs. The point is perhaps best illustrated by reference to one of Gleason's definitions. Two (or more) morphs are said to represent the same
3. Bloomfield 1935 p. 164 .
1. Gleason 1966. 
morpheme if they have 'some common range of meaning', and if they are 'in complementary distribution conditioned by some phonologic feature ${ }^{2}$. (Such a case would be the English noun plurals $/-z /, /-s /$ and $/-i z /$ ) It is clear that the enterprise of constructing a grammar here depends on being able to identify various morphs (e.g. $/-z /, /-s /, /-i z /$ ) as having the same meaning (say, 'more than one'); but provided we can do this we need not bother about any lack of phonological resemblance between the morphs in question.

Thus although the case is different from that of setting up M-units on the basis of 'phonetic-semantic resemblance', it is again the appeal to synonymy which supports the distinction between norph (or allomorph) and morpheme.

3.14 It may perhaps be questioned whether a proviso as strong as that of synonymity of the morphs in question is required in the cases under discussion. Would it not be sufficient to allow that e.g. $/-z /, /-s /$ and $/-i z /$, or duke and duch-, may differ in meaning, provided any such differences are granted to play no part in determining the selection of these morphs as elements in English sentences? We might conceive of these cases as ones in which, to adopt Hockett's words, the choice 'is made for the speaker rather than by him' ${ }^{1}$, or -we must add- if by him, then not as a semantic choice. This would allow the distinction between morph and morpheme to be drawn, and drawn on a semantic basis, but without invoking synonymy.

2. Gleason $1966 \$ 7.3$.

1. Hockett 1958 \$15.1. 
3.15 Along these lines, the concept of morpheme alternance might be explicated for purposes of semantically based morphology as the concept of the relation between members of a class of phonologically differing forms which share a common meaning, and between which the language affords the speaker no grammatically indeterminate semantic choice. (That is to say, either there is no choice (because by grammatical rule all members of the class but one are excluded), or, if more than one member of the class is grammatically permissible, then it makes no semantic difference which of those permitted is chosen.)

This appears to cover all the usual examples adduced in discussions of morphological analysis. And yet there is something very curious about this concept. It may be observed, first of all, that if we strengthen the first semantic requirement so that instead of speaking forms which 'share a common meaning' we speak of forms 'which are identical in meaning', then the second requirement becomes redundant, since if the forms have the same meaning, then ipso facto a speaker has no semantic choice. (We thus reintroduce the proviso of synonymity.)

On the other hand, it is difficult to see how the concept of alternance as stated could be of any practical use in morphological analysis unless supplemented by criteria for determining sameness of meanings. Attention is rarely paid to such criteria, and when it is the results are palpably inadequate. For example, the demonstration given by Hockett ${ }^{1}$ that the Bnglish noun plurals / $/ \mathrm{s} /$ and $/-z /$ have no discoverable difference in meaning is question-begging. What Hockett successfully demonstrates is

1. Hockett $1958 \quad \$ 15.1$. 
simply that they occur in complementary distribution. But it is hardly surprising that a substitution test of the kind Hockett uses should fail to discover a difference in meaning between forms in complementary distribution: on the contrary, it must fail. To take such a test seriously involves supposing that complementarity of distribution is a sufficient condition of synonymity. But anyone who supposed that could easily demonstrate that e.g. English the and 's are synonymous. Moreover, if such a test is to be treated as decisive, it would be simpler to stop talking about differences of meaning and simply rely on the test. For the test is applicable, irrespective of what we suppose it to be a test of. But normally no-one goes even to the trouble taken by Hockett to exhibit the supposed synonymy of allomorphs of the same morpheme: at best we are offered a brief identification of 'the meaning' they are said to share (e.g. 'more than one' for noun plurals) and for the rest it is simply assumed that any differences of meaning are morphologically irrelevant.

If we relied simply on the discovexy of a 'common meaning', however, we should be forced to conclude that e.g. knives and forks contained not one but two pairs of morphs with common meanings. For a common meaning can be stated for each pair (e.g. 'implement', 'more than one') and the grammatical rules of Inglish never offer a choice between the morphs in question (knive : fork and $/-z /: /-s /$ ). It is no objection to this example to point out that everyone knows that the plural of knife differs in meaning from the plural of fork. Doubtless they do differ, and this is the point of the example. What is illustrated thereby is that there 
is no way of saying just how much the two morphs must have semantically in common, short of the synonymity proviso.

3.16 To summarize, setting up synonymous morphs is necessitated in semantically based morphology to the extent that the morphophonemic rules of I produce alternations like $/-\mathrm{s} /, /-\mathrm{z} /$ and $/-i z /$, or 'irregularities' like good / better etc. If such phenomena occur, the postulation of synonymous morphs can be avoided in the last resort only by treating the word as the minimum unit, and not inquiring into its internal structure. Some semantic basis would still be needed for dealing with M-relations, but synonymities could be dispensed with. (Thus, for example, we would need some semantic basis for identifying the invariant which allows us to say that better is the comparative of good; but if we renounce interest in the bimorphic structure bett involve equating the meaning of good with that of bett-, any more than we need to be able to identify a segment of worse having the same meaning as bad.) Granted, however, that morphological description is - some would say by definition - committed to the analysis of words, it may be said that setting up synonymous morphs by way of accounting for word structure is ultimately determined by certain empirical facts about the surface grammar of natural languages.

3.2 If the objective of syntactic description is taken to be simply the specification of all and only the grammatical concatenations of morphs in the language, the question whether two such concatenations are synonymous, like all other questions about the semantic properties of grammatical sequences, falls outside the province of syntactic analysis. Questions of 
meaning become relevant, however, if we take it to be part of the function of a syntax to assign structural descriptions to the specified grammatical sequences. For it may be asked of any given sequence whether it has one or more than one grammatical analysis, and the fact that a sentence is or is not ambiguous is here of some importance. E.g. the fact that the German sentence Das ist natürlich genug is assigned two structural descriptions is directly related to the fact that it is ambiguous ${ }^{1}$. Transformational grammarians, following Chomsky, have normally included non-lexical ambiguities as being among the facts to be accounted for by an adequate grammar ${ }^{2}$, and in accounting for these have implicitly provided explanations for certain synonymities and non-synonymities as well ${ }^{3}$.

1. Bach $1964 \$ 3.3$ gives the analyses:

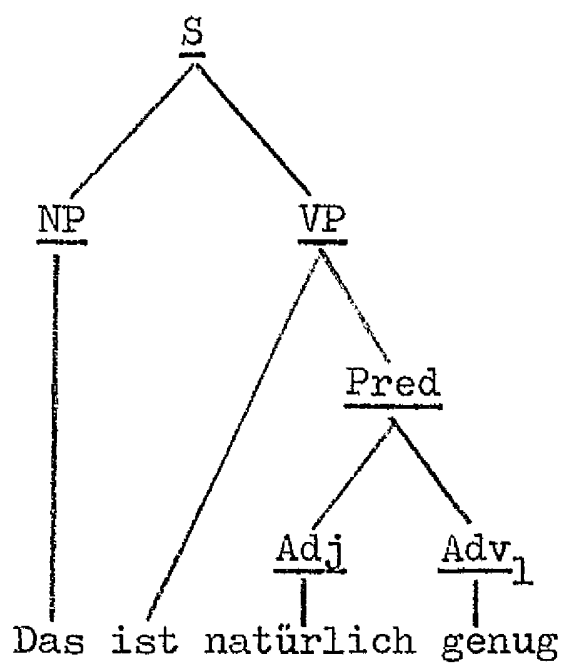

That's natural enough

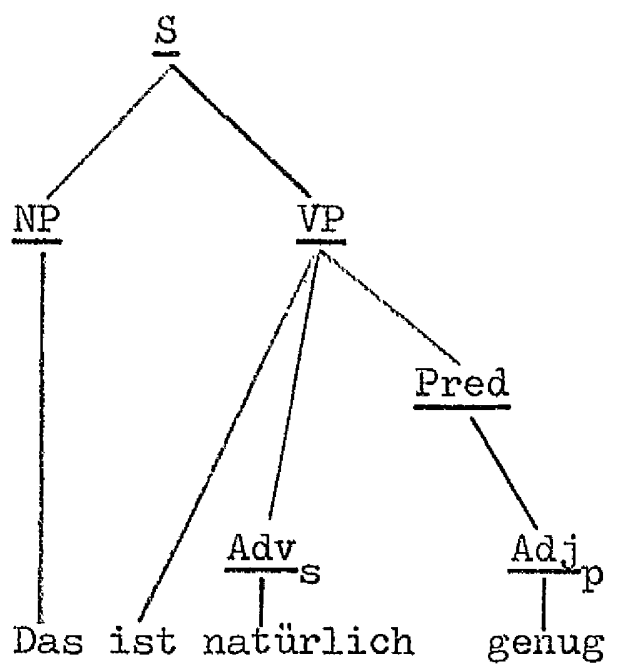

That's naturally enough

2. Chomsky $1957 \$ \$ 4.1$ and 8.1. Bach $1964 \$ 1.2$ and 5.2.

3. For example, the fact that, in soite of certain structural and lexical parallels, old men's shoes and men's old shoes do not receive identical semantic interpretations is very well accounted for by the grammatical ambiguity of the former. 
3.21 The relation between synonymy and grammatical ambiguity may conveniently be discussed by taking as a starting point the following proposition:

$$
\begin{aligned}
& \text { Al 'If axy and bxy are synonymous, then either both } \\
& \text { are grammatically ambiguous, or neither.' }
\end{aligned}
$$

That is to say, if the sequences $\underline{a}+\underline{x}+\underline{y}$ and $\underline{b}+\underline{x}+\underline{y}$ are both syntactic arrangements permitted by the grammar of the language, and we have a guarantee that the resultant expressions axy and bxy do not differ in meaning, then it cannot be the case that only one of these expressions is grammatically ambiguous.

Al appears to correspond to the basic position adopted by transformational grammarians, as witnessed by such statements as the following:

'Occasionally, a grammax may permit us to construct nonequivalent derivations for a given sentence. Under these circumstances, we say that we have a case of "constructional homonymity", and if our grammar is correct, this sentence of the language should be ambiguous ${ }^{1}$. 'Obviously, not all kinds of ambiguity will be analyzable in syntactic terms. For example, we would not expect a grammar to explain the referential ambiguity of "son" - "sun", "light" (in color, weight), etc. ${ }^{2}$

'We can test the adequacy of a given grammar by asking whether or not each case of constructional homonymity is a real case of ambiguity and each case of the proper kind of ambiguity is actually a case of constructional

\footnotetext{
1. Chomsky $1957 \oint 4.1$.

2. Chomsky 1957 8.1 n.1.
} 
homonymity ${ }^{3}$.

'We can state as a general requirement for a total theory of a language that any ambiguous sequence must have several representations in the theory. This requirement is quite parallel to the condition placed on a phonological theory that no two sequences that are "different" (i.e. consistently distinguishable by a pair test) may be represented phonemically in the same way. That is, beyond the phonological level, we can demand that no two sequences that are "different" may be represented in the same way, even if they happen to have the same phonemic shape. Some of these differences will be accounted for by a theory of language usage ... Some, presumably, will be accounted for by a semantic theory: Look at the table is ambiguous only because table has several meanings, e.g., 'mathematical table,' 'dinner table.' In the realm of grammar proper, different representations may exist ... on the level of phrase structure (different $P$ markers for the same string). They may also exist in the transformational level, as in ... I don't approve of his cooking, or John is crazy to go ${ }^{4}$.

In discussing $A$, it may be taken for granted that if, in the context '. $\underline{x y}$ ', a is lexically ambiguous but $\underline{b}$ is not, then axy and $\underline{\text { bxy }}$ will not be synonymous; but then the grammar is not expected to account for the ambiguity.

The case for believing Al to be correct - if it is correct - depends on the semantic implications of the term 'grammatical ambiguity', which must now be examined.

3. Chomsky $1957 \oint 8.1$.

4. Bach $1964 \oint 5.2$. 
3.22 Some clarification of the distinction between grammatical and lexical ambiguity is first necessary. It may be and often is the case that a grammatical ambiguity involves the assignment of two meanings to a single lexical item. For example, in $\underline{I ~ s a w ~ l o g s}^{1}$ the lexical item saw may be assigned two distinct meanings, but also two distinct grammatical classifications ('past tense' of see vs. 'present tense' of saw). Thus although the ambiguity centres about one particular lexical item, the grammatical analysis of the sentence containing it is affected. This is the justification for calling I saw logs a 'syntactical ambiguity'? : the sentence yields the two phrase markers

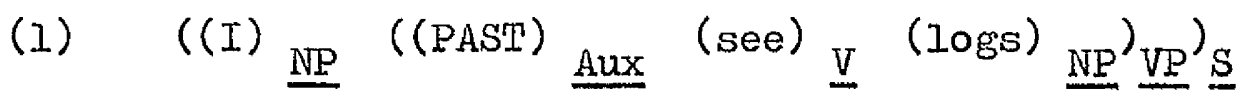

and (2) ((I) ${ }_{\mathrm{NP}}^{((\text {saw })} \underline{\mathrm{V}}^{(\text {logs })} \underline{\mathrm{NP}}^{))_{\mathrm{VP}}} \underline{\mathrm{S}}$

If we prefer to reserve the term 'syntactical ambiguity' for bracketing ambiguities (constructional homonymities), an alternative would be to call I saw logs a 'distributional ambiguity' since, as Iyons observes 3 , the ambiguity in such cases is a function of the distributional classification of the item involved. (Here saw belongs to two distributional classes, in one of which it has such co-members as heard, found, brought, and in the other such co-members as hear, find, bring.)

When 'Iexical ambiguity' is contrasted with 'grammatical ambiguity' the implication usually is that no grammatical factors are appealed to in

1. An example discussed by Katz \& Martin 1967 p. 484.

2. As do Katz \& Martin 1oc. cit.

3. Lyons $1968 \oint 6.1 .3$. 
explaining the ambiguity of the expression in which the lexically ambiguous item occurs. The connexion between lexical and grammatical (syntactical) ambiguity is analysed along these lines by Katz \& Postal as follows:

'A lexical item is ambiguous when it has more than one sense. Ambiguity at the lexical level is the source of semantic ambiguity at the sentence level. Thus, a necessary but not sufficient condition for a syntactically unambiguous sentence to be semantically ambiguous is that it contain at least one ambiguous lexical item. For example, the source of the semantic ambiguity in the sentence

(2) he enjoys wearing a light suit in the summer is the ambiguity of the lexical item light. Since an adequate dictionary entry for a lexical item must mark every one of its senses, the dictionary entry for light must represent it as at least two ways ambiguous, in terms of two readings which differ from each other in that one contains the semantic marker (Color) but not the semantic marker (Weight) and the other contains (Weight) but not (Color). Since there is nothing in (2) to exclude either one of these readings as genuine readings for the occurrence of light, the sentence is semantically ambiguous, one term of this ambiguity stemming from each of these readings.

However, the presence of an ambiguous lexical item in a syntactically unambiguous sentence is not a sufficient condition for that sentence to be semantically ambiguous. For example, although the sentence

(3) the stuff is light enough to carry ${ }^{4}$

4. Katz \& Postal's example here is poorly chosen, since it appears that

'... enough to carry' must be grammatically ambiguous: i.e. somewhere cont'd. 
contains an occurrence of the ambiguous lexical item light, it is not itself ambiguous because light enough to carry is not understood to mean 'Iight enough in color to be carried'. Thus when there is an ambiguous lexical item in a semantically unambiguous sentence, either the syntactic properties of the sentence or the meanings of the other constituents prevent the ambiguous lexical item from contributing more than one of its senses to the meaning of the whole sentence ${ }^{5}$.

The example of light illustrates the residual character of the notion of lexical ambiguity, being dependent on the assumption that the derivations for sentences containing light are identical in respect of the way in which they eventually produce the formative light. But a detailed. analysis of English might perhaps uncover grounds for assigning two syntactic markers to the lexicon entry for lijght (? 'Appearance Adj.' vs. 'Substance Adj.'), and then the need to consider light a case of lexical ambiguity would lapse.

3.23 An analysis which appears to allow the possibility of contradicting $A I$ (3.21) is offered by Lyons in his version of the distinction between grammatical and semantic ambiguity:

'Let us assume, for the sake of the argument, that neither fresh fruit market nor new fruit market has more than one interpretation:

in the history of the sentence there has occurred conflation which has obscured the modal difference between 'it is possible to carry $\underline{x}$ ' and 'it is desirable (advantageous, opportune, etc.) to carry $x$ '. Hence the sentence, contrary to Katz \& Postal's interpretation, can have the reading 'the stuff is light enough (in colour) to carry'.

5. Katz \& Postal 1964 p. 15. 
from the semantic point of view, we will say that they are not ambiguous. Are they grammatically ambiguous? Is the constituent-structure fresh (fruit market), in the one case, and (new fruit) market, in the other, grammatically acceptable? To answer these questions we must of course refer to some explicit grammar of English. . It is clear that, in general, the bracketing $\underline{A}+\left(\underline{N}_{1}+\underline{N}_{2}\right)$ is acceptable if the first noun can combine with the second (fruit market) and if the adjective can combine with the second noun (new market, ? fresh market); and the bracketing $\left(\underline{A}+\underline{N}_{1}\right)+N_{2}$ is acceptable if the first noun can combine with the second noun and if the adjective can combine with the first noun (fresh fruit, ? new fruit) ... Any phrase of the form $\underline{A}+\underline{N}_{-}+\underline{N}_{2}$ will be given two grammatical analyses, unless the grammar and the lexicon to which we refer prohibits explicitly the combination of the adjective in question with one or other of the nouns ${ }^{1}$.

Let us now develop Lyons's exarnple in the following way: first, by assuming fulfilment of the condition mentioned in Lyons's last sentence, namely that the grammar and lexicon explicitly prohibit the combination of the adjective with one (but not the other) of the nouns in question, and second, by stipulating not merely that fresh fruit market and new fruit market are semantically unambiguous but also that they are synonymous.

It would now follow from Lyons's argument that, unless there is a contradiction in the stipulations, we have a case where axy and bxy are synonymous but only one is gramnatically ambiguous.

1. Lyons 1968 6.1 .3$. 
That there is no contradiction in the stipulations may be concluded from the following considerations. I. If we were committed to supposing that the synonymy of fresh fruit market and new fruit market was the result of the independent synonymy of fresh and new, then it might be queried why one of these adjectives could qualify one but not the other of the nouns. But there seerns to be no general reason to deny that synonymous complex expressions may be made up of lexical items which, considered in isolation, or in other combinations, are not synonymous. The unambiguous and identical semantic interpretation of two phrases or sentences is not a sufficient condition for identity of the lexical entries of their corresponding lexical items. 2. There seems to be no general reason to deny that a lexical item which is grammatically permissible in a given context $C_{1}$ may not be so in a partially similar context $\mathrm{C}_{2} \cdot 3$. The stipulations made involve no stronger claims than those covered by the preceding two points, namely that (i) axy and bxy may be synonymous even if $\underline{\text { a }}$ and $\underline{b}$ are not, and (ii) axy and $\underline{b x y}$ may be grammatical when $\underline{a x}$, or $\underline{a y}$, or $\underline{b x}$, or by are not.

It now appears that by following Lyons's argument we have reached a conclusion which conflicts with $A$, and indeed this is so if the term 'grammatically ambiguous' in Al is to be given the sense which Lyons implicitly gives it in answering the question 'Are they grammaticaliy ambiguous?' .

But there are reasons for rejecting the way Lyons interprets this question, and hence also for rejecting the answer he gives. The main objection may be stated concisely as follows. Lyons assumes the question 
to mean 'Are they ambiguous according to a grammar?' and gives the trivial and unhelpful answer 'They are if the grammar says so'. Whereas if the question is to have any linguistic interest at all, it must mean 'What linguistic facts would lead one to construct a grammar which represented them as ambiguous?'. Iyons's answer - if it is read as an answer to that question - is perhaps less trivial, but now becomes baffling. For we are being told that the ungrammaticality of new fruit (in isolation) should preclude the assignment of grammatical ambiguity to new fruit market - without, however, being told why. But that is precisely what we want to know in order to clarify the notion of grammatical ambiguity ${ }^{2}$.

3.24 An alternative interpretation of 'grammatical ambiguity' is available, but it leans too heavily on semantic considerations to be acceptable to one who adopts Lyons's position. According to this alternative interpretation grammatical ambiguity is a property of certain types of construction, and any individual construction either belongs to a grammatically ambiguous type (in which case it counts individually as grammatically ambiguous) or it does not. On this view, the ungrammaticality of new fruit would not count against the grammatical ambiguity of new fruit market: for the question to be answered is not 'Is new fruit grammatical?' but 'Does new fruit market belong to a grammatically

2. Putting forward a solution for a particular example, in advance of any general principle, simply constitutes a retreat into obscurity, i.e. What is now unclear is what constitutes the incompatibility between the ungrammaticality of new fruit and the grammatical - as opposed to semantic - ambiguity of new fruit market, For it will hardly do to say that 'grammatical ambiguity of axy' just is 'independent combinability of $\underline{a}, \underline{x}$ and $\underline{y}^{\prime}$ ' 
ambiguous construction-type?', where 'grammatically ambiguous constructiontype' is defined as one in which semantically unambiguous elements may be combined into semantically ambiguous complexes.

To indicate the general reasons for adopting this definition (which places 'grammatical ambiguity' in a position of dependence on the prior notion of 'semantic ambiguity') it will be relevant to consider some grammatical ambiguities other than 'bracketing arnbiguities'. Lyons distinguishes two further basic types of grammatical ambiguity, exemplified by They can fish and amor Dei, the former being a case of what was above (3.22) termed 'distributional ambiguity' (can is both a modal auxiliary and a transitive verb, and fish both an intransitive verb and a noun), and the latter a case of 'transformational ambiguity' (Deus amat vs. Deum amat). ${ }^{1}$

It is relevant to the problem under discussion to note that in drawing a distinction between such cases as types of grammatical ambiguity, there is already a certain amount of illicit trading on the notion of 'ambiguity' - illicit, that is to say, if we wish to maintain that semantic ambiguity and grammatical ambiguity are independent notions.

For example, although it may be convenient to speak of 'grammatical ambiguity' in cases when a position which can be occupied either by an A-class form or by a B-class form is in fact occupied by a form which belongs to both classes, it must be realized that strictly from the point of view of distribution of forms it is nonsense to apply the term

1. Lyons 1968 $6.1 .3, \oint 6.6 .2$. 
'ambiguity'. For there is no sense in which a form must be either A-class or B-class (exclusive disjunction) to occur in such a position: we could equally well set up an $A B$-class limited to forms which occur both in A-class positions and in B-class positions, and the question whether a form occurring in a position open to both is an A-class form or a B-class form would then simply not arise. The term 'ambiguity' in such cases tacitly appeals to the fact that in sentences where an AB-class form occupies an A-class position it will often receive a different semantic interpretation from that which it receives when occupying a B-class position; with the result that when it occupies an AB-class position, one asks which semantic interpretation it should have. No-one, presumably, would speak of 'ambiguity' either (i) if a position is filled by a form which elsewhere occurs only in A-class (or only in B-class) positions, or (ii) if forms which may occur both in A-class positions and B-class positions receive exactly the same semantic interpretation in both types of position.

Or, to take a different type of case, if men and old women did not differ in meaning from old men and old women or from old men and women (i.e. if the adjective semantically applicable to both nouns could be syntactically preposed to either or to both) and if constructions of this type did not admit the interpretation whereby the adjective was semantically relevant to one noun only, then presumably no-one would regard old men and women as grammatically ambiguous. Yet it would remain true that old men and women could be treated as syntactically derived in more than one way (e.g. by deletion of the second old from old men and old women, or by preposition to men and women, or by fronting from men and old women). But if there is to be any point in speaking of 'ambiguity' there has to be 
some sense in which one construction subsumes the other two, and they are not just equal partners.

3.25 In general, it would seem that to maintain a distinction between grammatical and semantic ambiguity is legitimate only to the extent that knowledge of grammatical ambiguities may be explicated as non-semantic (i.e. formal) linguistic knowledge. The question how far this 'extent' goes requires some consideration.

Knowledge of the grammaticality or otherwise of a sentence or construction must be counted formal knowledge, since it is knowledge of the combination rules governing sets of formally defined items (i.e. the words or morphs of the language). The knowledge which enables a speaker to segment utterances into constituent grammatical units is also to be counted formal knowledge, since it can be treated as knowledge of substitution procedures not presupposing any knowledge of the meanings of the items involved. In short, all knowledge of the nouns, adjectives, verbs, etc. of a language and their rules of combination may be regarded as knowledge of what may be substituted for what and in what contexts. But whether this includes knowledge of grammatical ambiguities is not immediately obvious.

It might appear that in some instances at least a case can be made for analysing knowledge of grammatical ambiguity as knowledge of relations between substitution constraints. H.g. knowledge of the grammatical ambiguity of They can fish might perhaps be said to be knowledge that in this sentence can and fish may each be replaced salva grammaticalitate by members of two different sets of expressions related in a certain way. If 
we call these sets of expressions 'can-A', 'can-B', 'fish-A' and 'fish-B', the rule is that 'can-A' substitutes must go with 'fish-A' substitutes and 'can-B' substitutes with 'fish-B' substitutes'. Knowledge of the gramnatical ambiguity, it might be suggested, is simply knowledge of this rule, i.e. of a specific interdependence between the substitution possibilities for can and fish.

But grammatical ambiguity cannot simply be equated with interdependence between substitution patterns of morphs or words in a given sentence. For on the one hand there would seem to be cases of grammatically ambiguous sentences where no relevant interdependence obtains, while on the other hand there are cases of interdependence which do not correspond to grammatical ambiguity. An example of the former would be I saw logs, and of the latter John bought it. In the former instance, the grammatical ambiguity of saw (present tense of saw vs. past tense of see) matches no interdependence between the permissible substitutions

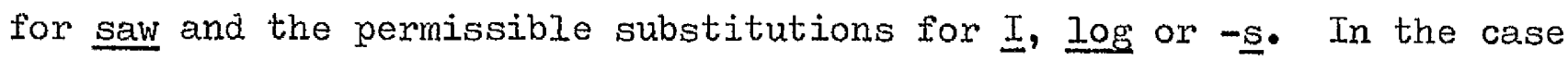
of John bought it, the following interdependence holds between substitutions for bought and substitutions for it: 'bought-A includes ate; 'bought-B' includes drank; 'it-A' includes bread; 'it-B includes wine. This rule expresses the fact that we cannot have "John drank bread, nor *John ate wine. Thus there is here a substitutional interdependence, but it does not seem to correspond to a grammatical ambiguity in John bought it.

1. 'Can-A' will include may, could, will,...; ' can-B' will include pack, sell, take,...; ' fish-A will include go, come, stay,...; 'fish-B' wili include peas, beans, tomatoes, 
More generally, a major difficulty in the way of explicating grammatical ambiguity in terms of membership of substitution sets is that each word in the language may be regarded as belonging to many overlapping substitution sets. The approach thus leads ultimately to the proposition that if a word belongs to $\underline{n}$ different substitution sets in different contexts, and there is a context in which it may be replaced by any member of any of its $\underline{n}$ substitution sets, then in that context the word is $\underline{n}$-ways grammatically ambiguous. Similarly, a word would be $\underline{n}$-minus-x-ways granmatically ambiguous in contexts where it may be replaced by any member of $\underline{n-m i n u s-x}$ of its substitution sets, and unambiguous where n-minus- $\underline{x}$ equals one. But since almost every sentence in every natural language would then turn out to be multiply gramatically ambiguous, this is presumably an account of 'grammatical ambiguity' which no-one would readily accept.

3.26 An alternative approach to the explication of grammatical ambiguity is by reference to an explicit set of generative rules for producing the sentences of the language. Then a sentence or constituent is unambiguous if the rules give only one way of generating it, and $\underline{n}$-ways gramatically ambiguous if the rules give $\underline{n}$ ways of generating it. However, this account of grammatical ambiguity depends on there being guarantees that the rules do not contain either 'superfluous' ways of generating particular sentences, or 'insufficient' ways of generating particular sentences. But since the tests of 'superfluity' and 'insufficiency' are semantic 
tests ${ }^{l}$, this approach does not enable us to account for grammatical ambiguity without appeal to meanings.

The plausibility of a semantically based interpretation of grammatical ambiguity gains support from the difficulty of finding convincing counterexamples, i.e. intuitively clear cases of grammatical ambiguity where no systematic semantic ambiguity is generated. Examples which come to mind in this connexion - e.g. that of recursive co-ordinate constructions seem to be cases in which any alleged 'grammatical ambiguity' is a product of the convention adopted for representing the grammar, and corresponds to no genuine item of linguistic knowledge at al1 ${ }^{2}$.

3.27 The above considerations weigh in favour of explicating grammatical ambiguity in terms of capacity to generate semantic ambiguities. Accordingly, the question whether fresh fruit market and new fruit market are grammatically ambiguous even if semantically unambiguous becomes the question whether the combination $A+N+N$ is one able to combine seman-

1. cf. 3.21 n.3. The 'insufficiency' of rules which give only one way of generating old men's shoes is demonstrated by the fact that old men's shoes has two distinct semantic interpretations, given only one meaning each for old, men's and shoes. The 'superfluity' of rules which give fourteen different ways of generating old men's shoes is demonstrated by the fact that old men's shoes does not have fourteen distinct semantic interpretations.

2. A grammar's ambiguous representation of old men's shoes corresponds to a genuine item of linguistic knowledge in that the competent speaker-hearer knows that old may go either with men or with shoes, whereas it would be nonsense to say that a grammar's ambiguous representation of Tom and Dick and Harry corresponds to the competent speaker-hearer's knowledge that Dick may go either with Tom or with Harry. 
tically unambiguous items into semantically ambiguous complexes. This question will receive an affirmative answer if suitably ambiguous expressions of the type $A+N+N$ can be found, where the ambiguity corresponds to the possibility of bracketing $(A+N)+N$ or $A+(N+N)$, the same meanings being assigned to individual words in either case ${ }^{1}$.

Interpreting Al in this sense, Al states that if axy and bxy are synonymous, then it cannot be the case that only one of them belongs to a construction-type able to combine semantically unambiguous items into semantically ambiguous complexes. Now for this to be so, we have to take the assurance of synonymity to preclude the possibility that one expression but not the other instantiates a grammatical ambiguity which in this instance happens to be semantically inconsequential. An assurance merely of the 'synthetic synonymity' of axy and bxy (i.e. an assurance that the two expressions command the same semantic interpretation as wholes, irrespective of their internal structure) is insufficient to validate $A$ I in the light of the interpretation given above of 'grammatical ambiguity'. For it might be the case that a but not $\underline{b}$ belongs to a class of words which combine grammatically with members of the class to which $\underline{x}$ belongs, but that in this instance 'a $+\underline{x}$ ' has a meaning which, combined with the meaning of $y$, yields the same meaning ' $M$ ' for the whole expression axy as is independently yielded by combining the meaning of a with that of ' $x+y$ '. In such a case, the possibility of interpreting

1. Such expressions exist in English, e.g. red wine punch, foreign book shop. 
axy grammatically either as (ax)y or as $\underline{a}(\underline{x y})$ is semantically of no consequence, since axy means ' $M$ ' in either case.

The guarantee of 'synthetic synonymity' between $\underline{\text { axy }}$ and $\underline{b x y}$, (here the guarantee that axy and bxy have only the meaning 'MI') thus does not ensure parity of grammatical ambiguity between them.

Can we, then, formulate a stronger condition which will validate Al? 3.28 We may usefully explore this question by drawing on the theoretical apparatus of generative grammar. Of particular relevance is the distinction of Katz \& Postal between sentence and sentoid (the latter defined by reference to a semantically interpreted P-marker) ${ }^{I}$. Taking axy and bxy as sentences, the possibility we are attempting to guard against is the possibility that axy (but not bxy)represents two sentoids, each of which receives the same derived reading as that for the sentoid corresponding to bxy. This possibility would be instantiated by a case ${ }^{2}$ in which axy had only the P-markers

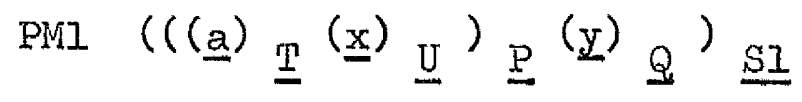

1. Katz \& Postal 1964 p. 27.

2. The relevant P-markers may be diagrammed as follows:
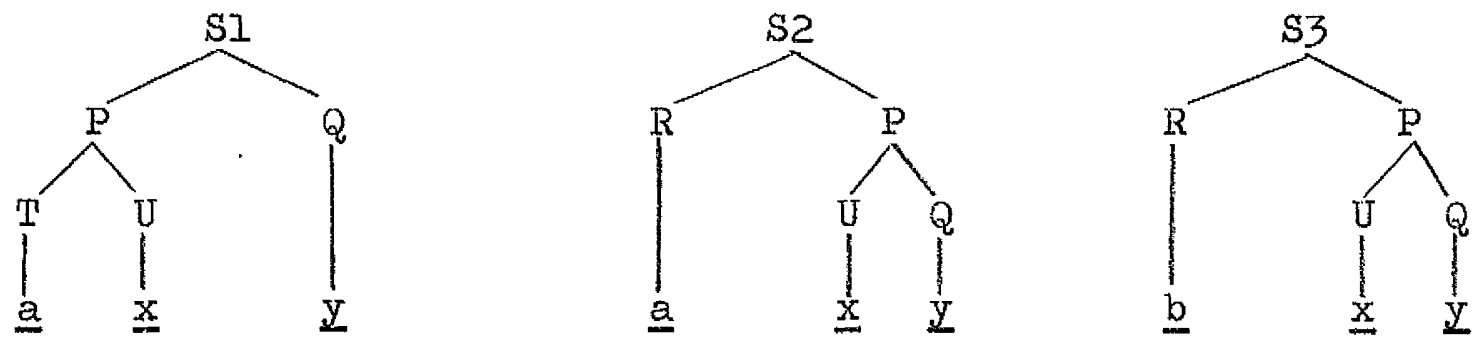
and PM2 $\left.\quad(\underline{a})_{\underline{R}}\left((\underline{x})_{\underline{U}}(\underline{y})_{\underline{Q}}\right)_{\underline{P}}\right)^{\underline{S 2}}$

and bxy only the P-marker

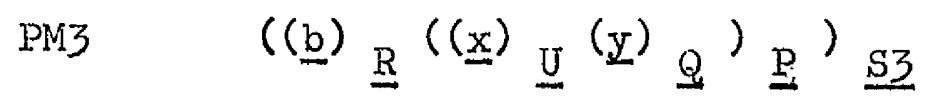

and where SI, S2 and S3 were full paraphrases (fully synonymous) under Katz \& Postal's definition

D5 $C$ and $C^{\prime}$ are fully synonymous with respect to $P M$ and $\mathrm{PM}^{\prime}$ iff and only if the set of readings associated with the node labeled ' $\mathrm{C}$ ' in $\mathrm{PM}$ and the set of readings associated with the node labeled ' $\mathrm{C}$ '' in $\mathrm{PM}^{\prime}$ are identical; $\mathrm{PM}$ may equal $\mathrm{PM}^{\prime} .^{3}$

But there is another possibility which would be equally damaging to Al. This is the possibility that axy (but not bxy) represents two sentoids, one of which happens to be semantically anomalous under Katz \& Postal's definition

D1 C is semantically anomalous with respect to PM if and only if the set of readings associated with the node labeled ' $C$ ' in PM contains no readings, i.e. is null. 4 and the other of which receives a derived reading identical with that of the sentoid corresponding to bxy. This would be the case, in terms of the example just given, if in PMl the node $Q$ had a reading which failed to combine with the derived reading for $P$, and $S 2$ and $S 3$ were fully synonymous under D5.

3. Katz \& Postal 1.964 p. 27.

4. Katz \& Postal 1964 p. 26. 
The assurance of what was referred to in 3.27 as the 'synthetic synonymity' of axy and bxy may be reformulated in Katz \& Postal's terminology as an assurance that all pairs of nonanomalous sentoids for axy and bxy respectively are fully synonymous (are 'full paraphrases') and there is at least one such pair. But this condition is not strong enough to validate Al because of the possibility of counterexamples of the kind just discussed.

The stronger condition which must be met to eliminate such counterexamples is clearly that axy and bxy should have the same number of sentoids and that it should be possible to put their respective sentoids in one-one correspondence, each such pair being fully synonymous under D5. Sentences (and, by extension, constituents of sentences) which meet this condition might be termed 'structurally synonymous'.

If an assurance of the synonymity of axy and bxy is taken to be an assurance of their 'structural synonymity' as defined above, then AI is correct.

3.3 We are thus led to draw a distinction which it would have been irrelevant to introduce into a discussion of the role of synonymity statements at the phonological level, but which assumes importance in connexion with grammatical analysis.

It is not the only distinction of which this is true. Another is that between synonymous sentences which have different underlying Pmarkers and those which have the same underlying P-marker(s).

(1a) He brought home some furze

(Ib) He brought home some gorse 


\section{(2a) He wrapped the parcel up \\ (2b) He urapped up the parcel}

If we assume the above pairs are synonymous pairs, then (Ia) and ( $\mathrm{lb}$ ) are 'structurally synonymous' in the sense of 3.28, i.e. they represent two fully synonymous sentoids. (2a) and ( $2 \mathrm{~b})$, on the other hand, presumably come not from underlying P-markers which happen to coincide in their semantic interpretation but from one and the same P-marker. Such pairs may be said to be 'intrinsically synonymous'.

Pairs like ( $2 a$ ) and (2b) are related by optional singulary transformations as defined by Katz \& Postal:

'In earlier treatments of transformational grammar, such as Chomsky's in Syntactic Structures, optional singulary transformations had at least two distinct functions. First, they derived various distinct sentence types, questions, imperatives, negatives, etc., from one underlying declarative type. They thus had a substantive role in explaining differences in cognitive meaning between sentences of the same syntactic type. Second, singulary transformations related optional variants that were full paraphrases. These were intuitively stylistic variants of each other like

(133) a. all the men are married

b. the men are all married

c. he found out the truth

d. he found the truth out

In our discussion of the syntax of questions, imperatives, etc., 
we have given a great deal of support for a conception in which only the second function of singulary transformations survives. It therefore seems reasonable to say in general that the different outputs produced by optional singularly transformations are merely stylistic variants necessarily having the same cognitive meaning. Thus there can be a uniform characterization of the function of optional rules for both the syntactic and phonological components; such rules derive what is referred to in linguistics as free variation, and nothing else ${ }^{1}$.

It may be noted that intrinsic synonymity of expressions is not the guarantee required to validate $A l$, but rather one condition which would validate

A2 If abcd and abdc are synonymous, then either both are grammatically ambiguous, or neither.

We do not, however, need a guarantee of intrinsic synonymity to rule out e.g. the possibility that abdc might, unlike abcd, be open to the bracketing $\underline{a}(\underline{b d})$. For that purpose structural synonymity of abcd and abdc would meet the requirement. Thus intrinsic synonymity of the two expressions is a sufficient but not a necessary condition for validation of A2.

1. Katz \& Postal $1964 \mathrm{pp} .112-113$. In connexion with 'free varjation', however, it should be noted that whereas in syntax the truth of the statement that $a$ and $\underline{b}$ are synonymous is a necessary condition of the correctness of treating a and $\underline{b}$ as 'free variants' (in the sense of being 'merely stylist $\overline{i c}$ variants of the same sentence'), the same statement assumes, at the phonological level, the incorrectness of treating the phonetic realizations of $\underline{a}$ and $\underline{b}$ as free variants. 
3.31 The much discussed ${ }^{1}$ synonymy of actives and their corresponding passives may be subsumed under 'intrinsic synonymy', although a case can be made out for treating e.g.

\section{(3a) John kissed Mary}

and (3b) Mary was kissed by John

as having different underlying P-markers, which differ only in ways that are 'semantically insignificant ${ }^{2}$. This can be accommodated by modifying the definition of 'intrinsic synonymy' so as to include pairs of expressions having the same underlying P-marker(s) or 'noncontrastive' P-markers.

The question of deciding which pairs of sentences stand in an active-passive relation may be compared to the question earlier discussed (3.112 - 3.114) of determining M-relations. No difficulty arises when there is a cleax formal correspondence, as in cases such as (3a) and (3b). But there may be instances which are more like the go/went problem, as e.g. if $(3 a)$ and

1. Chomsky 1957 9.2.7, Katz \& Postal 1964\$4.2.1, Ziff 1966, Katz \& Nartin 1967.

2. Katz \& Postal 1964 p. 73. Katz \& Postal propose to treat passives as deriving from 'underlying P-markers containing an Adverb manner constituent dominating by plus a passive morpheme dummy' and not from the P-marker underlying the corresponding active (Katz \& Postal 1964 p. 72). Cf. Katz \& Martin 1967 p. 480, where 'sentences with the same underlying phrase marker or underlying phrase maxkers that are the same, except for elements that do not bear meaning' are said to be synonymous. The treatment proposed assumes that one of the 'semantic properties of dummy morphemes' is that they are assigned a null reading (Katz \& Postal 1964 p. 73). 


\section{(3c) Mary was bussed by John}

were graminatical, but $(3 b)$ and

\section{(3d) *John bussed Mary}

were ungrammatical. Here buss could be treated as the 'passive of' kiss. If, however, we have a situation in which (3a), (3c) and (3a) are grammatical but (3b) ungrammatical, or (3a), (3b) and (3c) are grammatical but ( $3 d$ ) ungrammatical, the resolution of the choice between setting up one P-marker or two is less clear ${ }^{3}$.

It would of course be a complete confusion to appeal to the synonymity of buss and kiss as a 'reason' for settling the issue one way or the other. For their synonymity is assured whatever the solution. This is simply another way of putting the point that in grammatical analysis a synonymity statement about particular morphs may correspond either to the structural synonymity or to the intrinsic synonymity of pairs of sentences in which they occur, and this is here a relevant difference (whereas for purposes of phonological analysis it is irrelevant).

3.4 Both 'structural synonymy' and 'intrinsic synonymy' must be distinguished from 'analytic synonymy', which is a term we may reserve for describing part-to-part semantic correspondence of expressions which are, as wholes, synthetically synonymous. Thus to the extent that synonymous complex expressions are composed of semantically equivalent

3. There will doubtless be other considerations which weigh in favour of or against treating buss as an independent lexical item, e.g. whether or not we can say There was a lot of bussing and cuddling at the party. 
morphs and grammatical arrangements of morphs, we shall say they are analytically synonymous. The pairs cited in 3.3

(Ia) He brought home some furze
(Ib) He brought home some gorse

and (2a) He wrapped the parcel up

(2b) He wrapped up the parcel

are analytically synonymous in this sense, the former pair being also structurally synonymous, and the latter pair intrinsically synonymous. Each morph and construction in (1a) can be matched with a semantically equivalent morph and construction in ( $1 b)$, and similarly for (2a) and (2b). But

(4a) John likes his unmarried aunt

and (4b) John likes his spinster aunt

even if (let us assume ${ }^{l}$ ) structurally synonymous, are not analytically synonymous throughout in that spinster does not match un+marri+ed.

3.41 Where morphological analysis is concerned, the distinction between synthetic and analytic synonymy often answers to the difference between forms which are determinate with respect to segmentation and those which are not. Thus suppose we have a noun zog which has a regular plural by addition of $\mathbf{- s}$ and also an irregular plural without parallel elsewhere in the vocabulary; so that we say one zog but either two zogs or two zigo. Then zogs and zigo are synthetically synonymous but not analytically synonymous, since whereas the meaning of zogs can be treated as the meaning

1. Doubtless there is in fact a semantic difference, since John's unmarried aunt might be a young girl. 
of zog plus the meaning 'more than one' of the suffix $-\underline{s}$, the meaning of zigo is not comparably analysable in terms of the meanings of constituent parts. Por in the case of morphs which are not determinate with respect to segmentation we cannot say what the constituent parts are. 3.42 Where syntactic analysis is concerned, analytic synonymity is a stronger condition of semantic equivalence than structural. synonymity, as may be shown by comparing the requirements in terms of Katz \& Postal's D5 (3.28). That is to say, for two unambiguous sentences to be analytically synonymous it will have to be the case not only that they represent sentoids satisfying D5, but additionally that D5 hold for every corresponding pair of constituents dominated by $C$ and $C^{\prime}$ respectively, without residue, i.e. the pair of sentoids must match structurally in respect of occurrence of nodes. Thus in
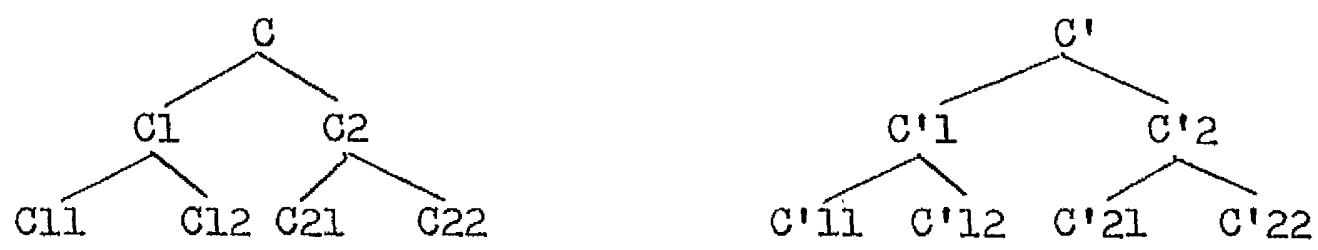

there will be structural synonymity if $D 5$ is satisfied for $C$ and $C^{9}$, but analytic synonymity only if D5 holds for each of the pairs $C$ and $C^{\prime}, C 1$ and $\mathrm{C}^{\prime} 1, \mathrm{C} 2$ and $\mathrm{C}^{\prime 2}$. Furthermore, the readings for the matching pairs of ultimate constituents CII and C'II, C12 and C'12, C21 and C'21, C22 and C'22 must be the same. But the conditions cannot be satisfied for e.g.
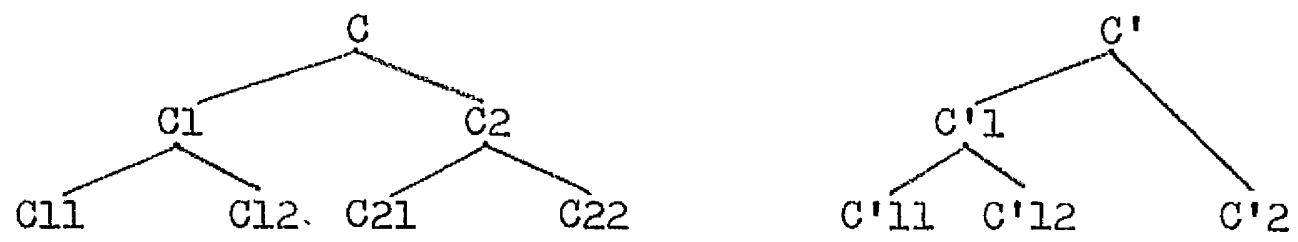

since $\mathrm{C} 21$ and $\mathrm{C} 22$ cannot be put in correspondence with anything under $C^{\prime}$. 
Accordingly $\mathrm{C}$ and $\mathrm{C}^{\prime}$ do not satisfy our requirement unless for any constituent $(\mathrm{Cl}, \mathrm{C} 2 \ldots)$ immediately dominated by $\mathrm{C}$ there is a corresponding constituent ( $\left.C^{\prime} I, C^{\prime} 2 . ..\right)$ immediately dominated by $\mathrm{C}^{\prime}$ such that the pairs of constituents ( $\mathrm{Cl}$ and $\mathrm{C}^{\prime} 1, \mathrm{C} 2$ and $\mathrm{C} \cdot 2, \ldots$ ) each satisfy $\mathrm{D}$, and similarly for constituents immediately dominated by those, until the ultimate constituents are reached.

3.43 The operation of a semantic component in transformational grammar does not normally allow for the distinction between synthetic and analytic synonymy to apply to sentences, since projection rules are cumulative, i.e. include an erasure proviso. Thus amalgations of readings will be accomplished by a rule such as:

$'\left(\underline{R}_{1}\right)$ Given two paths of the form

(1) Lexical String $\rightarrow$ syntactic markers of head $\left(\underline{a}_{1}\right) \rightarrow\left(\underline{a}_{2}\right) \rightarrow \ldots \rightarrow\left(\underline{a}_{n}\right) \rightarrow[1]$ <set of strings of markers $\underline{-1}>$

(2) Lexical String $\rightarrow$ syntactic markers of modifier $\rightarrow\left(\underline{b}_{1}\right) \rightarrow\left(\underline{b}_{2}\right) \rightarrow \ldots \rightarrow\left(\underline{b}_{m}\right) \rightarrow[2]<$ set of strings of markers $\underline{\Omega}_{2}>$

such that there is a substring $\sigma$ of the string of syntactic or semantic head markers and $\underline{Q} \mathbb{E} \underline{\Omega}_{2}$. There is an amalgam of the form

$$
\begin{aligned}
& \text { Lexical String } g_{2}+\text { Lexical String }{ }_{1} \rightarrow \text { dominating } \\
& \text { node marker } \rightarrow\left(\underline{a}_{1}\right) \rightarrow\left(\underline{a}_{2}\right) \rightarrow \ldots \rightarrow\left(\underline{a}_{n}\right) \rightarrow\left(\underline{b}_{1}\right) \rightarrow\left(\underline{b}_{2}\right) \rightarrow \\
& \ldots \rightarrow\left(\underline{b}_{m}\right) \rightarrow[[2][1]]\left\langle\underline{\Omega}_{1}\right\rangle,
\end{aligned}
$$


where any $\underline{b}_{i}$ is null when $\left(\exists \underline{a}_{i}\right)\left(\underline{b}_{i}=\underline{a}_{i}\right)$ and $[[2][1]]$ is [1] when $[2]=[1] \cdot 11$

Katz \& Fodor comment as follows:

The Iimiting case, where the addition to the compound of semantic material from the modifier is zero, is of considerable theoretical significance. The compound unmarried bachelor is a case in point. The erasure clause in $\left(\underline{R}_{1}\right)$, i.e., "any $\underline{b}_{1}$ is null when $\left(\exists \underline{a}_{i}\right)\left(\underline{b}_{i}=\underline{a}_{i}\right)$ and. $[[2][1]]$ is $[1]$ when $[2]=[1], "$ tells us to delete from the path of the modifier any semantic material already represented in the path of the head. Thus, in forming the compound unmarried bachelor all the semantic information in the path of the modifier unmarried will be deleted so that the derived path for unmarried bachelor will contain no more than the semantic material which comes from the path for bachelor. The failure of the modifier to add semantic information would appear to account for the intuition that such expressions as unmarried bachelor are redundant and that, correspondingly, such statements as "Bachelors are unmarried" are empty, tautological, vacuous, uninformative. 2

Katz \& Postal give a similar formulation for amalgamation by projection rule 3 and defend it by asserting that 'it makes no sense to include the semantic markers (Human) and (Pemale) twice in the reading associated with the compound expression spinster aunt just because each of the readings combined contains occurrences of both these markers. In

1. Katz \& Todor 1963 p. 507.

2. Katz \& Fodor 1963 p. 509.

3. Katz \& Postal 1964 p. 21. 
the derived reading for spinster aunt one occurrence of these markers is sufficient; another occurrence of each adds no semanicic information ${ }^{4}$. What derived readings miss in this way is the fact that pairs like spinster aunt and unmarried aunt are, if synonymous, synthetically but not analytically synonymous. If we substitute for the first word in unmarried aunt the word spinster we produce a phrase synthetically synonymous with unmarried aunt; but we have not done it by substituting a synonym for unmarried. This is indeed implicit in the elimination of the 'redundant' semantic information by the projection rule in the case of spinster aunt; but the derived reading no longer distinguishes between various ways in which the 'nonredundant' information represented by the reading has been accumulated. Thus all the synonymy definitions associated with the semantic component are in effect definitions of synthetic synonymy.

It follows that differences between analytically and synthetically synonymous constituents are not allowed to contribute to the derived reading(s) of the sentences in which they occur. But this is a mistake. For it takes no account of the distinction normally drawn by logicians between two kinds of semantic equivalence. Analytic synonymy, as the term has been used here, corresponds to what is sometimes called 'intensional isomorphism', or else appears as one of two possible varieties of 'equivalence in analytic meaning'.

Carnap gives the following account of intensional isomorphism: 'Let us consider, as an example, the expressions $12+5$ ' and 'II sum $V$ ' in a language $S$ containing numerical expressions and

4. Katz \& Postal 1964 p. 28 n. 10. 
arithmetical functors. Let us suppose that we see from the semantical rules of $\underline{S}$ that both ' + ' and 'sum' are functors for the function Sum and hence are Imequivalent; and, further, that the numerical signs occurring have their ordinary meanings and hence '2' and 'II' are I-equivalent to one another, and Iikewise ' 5 ' and 'V'. Then we shall say that the two expressions are intensionally isomorphic or that they have the same intensional structure, because they not only are I-equivalent as a whole, both being I-equivalent to '7', but consist of three parts in such a way that corresponding parts are I-equivalent to one another and hence have the same intension. Now it seems advisable to apply the concept of intensional isomorphism in a somewhat wider sense so that it holds also between expressions like ' $2+5$ ' and 'sum (II,V)', because the use in the second expression of a functor preceding the two argument signs instead of one standing between them or of parentheses and a comma may be regarded as an inessential syntactic device. Analogously, if '>' and ' $\mathrm{Gr}$ ' are L-equivalent, and likewise ' 3 ' and 'III', then we regard '5>3' as intensionally isomorphic to 'Gr(V,III)'. Here again we regard the two predicators ' $>$ ' and 'Gr' as corresponding to each other, irrespective of their places in the sentences; further, we correlate the first argument expression of ' $>$ ' with the first of 'Gr', and the second with the second. Further, ' $2+5>3$ ' is isomorphic to 'Gr sum (II,V), III', because the corresponding expressions ' $2+5^{\prime}$ and 'sum (II,V)' are not only I-equivalent but isomorphic. On the other hand, '7>3' 
and 'Gr surn (II,V), III' are not isomorphic; it is true that here again the two predicators ' $>$ ' and 'Gx' are I-equivalent and that corresponding argument expressions of them are likewise I-equivalent, but the corresponding expressions ' 7 ' and 'sum $(I I, V)$ ' are not isomorphic. We require for isomorphism of two expressions that the analysis of both down to the smallest subdesignators lead to analogous results ${ }^{5}$.

Lewis specifies two conditions under which expressions are equivalent in analytic meaning: either

'(1) if at least one is elementary and they have the same intension, or (2) if, both being complex, they can be so analysed into constituents that (a) for every constituent in either, there is a corresponding constituent in the other which has the same intension, (b) no constituent distinguished in either has zero intension or universal intension, and (c) the order of corresponding constituents is the same in both, or can be made the same without alteration of the intension of either whole expression ${ }^{6}$.

Condition (I) would include cases of synthetic synonymity, while condition (2) would include only cases of analytic synonymity.

The use which can be made of the distinction - as e.g. in Carnap's solution of Moore's paradox of analysis ${ }^{\text {? }}$ - affords ample evidence of its importance, and at the same time of the inadequacy of the transformationalist.

5. Carnap $1947 \$ 14.1$.

6. Lewis 1944 p. 246.

7. Carnap $1947 \$ 15.1$. 
concept of 'synonymy' (='synthetic synonymy'). For the transformationalist who operates with a semantic component as described by Katz, Fodor and Postal must say that the sentences

(6) A brother is a brother

and

(7) A brother is a male sibling

although having different P-markers are 'fully synonymous' (are 'full paraphrases'), and he is thus unable to explain how it is that these sentences have different communicational uses, and in particular how it is that (6) is totally uninformative whereas (7) is not. This difference cannot be attributed to the difference in form between (6) and (7), and must therefore be attributable to a difference between the meanings of the sentences. Yet, according to the transformationalist's account, there will be no difference between the meanings of the sentences.

Such examples point clearly to the need for distinguishing between two quite different implications of synonymity statements in a way which is, again, irrelevant at the level of phonological analysis.

3.5 The notion of 'intrinsic synonymy' (3.3) raises the question of how, ultimately, a distinction is to be drawn between items which just 'happen' to be synonymous, and those whose surface synonymity reflects an underlying identity at some more abstract level; as e.g. when we consider why one should treat pairs like

John kissed Mary

and Mary was kissed by John

as coming from the same or noncontrastive P-markers, but not pairs like John pleased Mary

and Mary liked John. 
It may be - and has been - regarded as a defect in Chomskyite transformational grammar that pairs of the latter type are not treated as transformationally related in a way that derives them from a common deep structure. Both pairs appear to be synthetically synonymous, and in a way which is systematically related to the synonymity of many parallel pairs of sentences in English.

A proposal which merits consideration in the present context is the following:

A3 In $\underline{\operatorname{axy}}$ and $\underline{\mathrm{bxy}}$, $\underline{\mathrm{a}}$ and $\underline{\mathrm{b}}$ are synonymous if whatever difference in meaning there may be between $\underline{a x y}$ and bxy is entirely determined by a difference in relations between deep and surface structure of axy and bxy.

Thus, for example, if $\underline{a}$ and $\underline{b}$ are verbs, and the semantic difference between axy and bxy resides in the fact that the 'logical subject' of axy is also:the 'grammatical subject' of the sentence, whereas the 'logical subject' of bxy is not, then it becomes possible to regard that difference as automatically determining the choice between $\underline{\mathrm{a}}$ and $\underline{\mathrm{b}}$ if $\underline{\mathrm{a}}$ and $\underline{\mathrm{b}}$ make no other independent contributions to the meanings of axy and bxy, and if these deep structure/surface structure relations are characteristic for sentences containing $\underline{a}$ and $\underline{b}$.

3.51 In Fillmore's elaboration of the theory of case grammar, it is proposed to treat the relations between various pairs of verbs as under A3. Each sentence is envisaged as having a 'case frame' which specifies the 'array of cases' the sentence provides. Case frames are represented in square brackets, with 'underline' indicating the position of the 
element with respect to which the expression is an environmental frame; e.g. the frame [ A] is one into which the verb run may be inserted, 'A' standing for the Agentive case and specifying the requirement of a 'typically animate perceived instigator of the action identified by the verb'l . Verbs are envisaged as having in their lexical entries 'frame features' which indicate the set of case frames into which the verb in question may be inserted. Frame features are represented in square brackets with ' + ' or ' - ' in front, indicating that the set of case frames represented by the expression within the brackets is that which will (if the feature is marked ' + ') or which will not (if the feature is marked ' -1 ) accept the lexical item in question. Parentheses indicate optional choices, and linked parentheses the obligatory inclusion of at least one of the elements so linked. Thus the verb kill has the frame feature ' $+[\ldots[(I / A)]$ ' specifying the requirement of 'An animate being affected by the state or action identified by the verb', and either an 'inanimate force or object causally involved in the action or state identified by the verb', or a 'typically animate perceived instigator of the action identified by the verb', or both. Verbs are regarded as being distinguished one from another not only in respect of the case frames into which they can be inserted, but also in respect of their transformational properties, including the selection of particular NPs to become surface subject or surface object of the verb.

1. Fillmore $1968 \mathrm{pp}$. 24-25. Other cases are: Instrumental ('I'), Dative ('D'), Factitive ('F'), Locative ('L') and Objective ('O'). 
When sentences are analysed in this way, it is feasible to show, according to Fillmore, 'that some syntactically different words are in fact semantically identical (with respect to that aspect of their meanings which is independent of the contribution of the associated cases) ${ }^{2}$. Thus like and please 'may be described as being synonymous. Wach has the frame feature +[ $\left.0+D^{\prime}\right]$; they differ only in their subject selection features. The verb like, in fact, has in its history the subject selection feature possessed by please ${ }^{2}$.

2. Fillmore 1968 p. 30 . According to Moore, the situation is somewhat more complicated than Fillmore allows for. Citing the examples

(1c) I liked the play

(Id) The play pleased me

(6a) The children pleased Sue yesterday by making theix beds

(6b) Sue liked the children yesterday for making their beds

Moore observes: 'I believe (6a) is ambiguous between the reading that Sue was pleased by the children where the children are in the same case relation, OBJECT, to Sue as, in (Id), play is to me and on the other hand the reading that the children are not in the case relation dominating the play; but are dominated by AGEN and thus are assigned the reading: animate responsible source of the action identified by the verb. Evidence that $(6 a)$ is indeed ambiguous is provided by sentences such as

the children set out to please Sue the children set out to like sue

where the first, but not the second, seems a clear case of an ordinary sentence needing no special interpretation. This much additional evidence suggests that please requires in its case frame the contextual feature $+[\quad$ OA] with the further constraint that the OBJECI selected must be [+ human], an object selection feature that does not apply to Iike' (Moore 1970). 
3.52 On this basis, a classification of lexical items may be drawn up, depending on the various possible ways in which synonymy combines with variation of frame features and other grammatical differentiae. Like and please will belong to the class of synonyms showing identical frame features. Show and see, on the other hand, will be synonyms which contrast in presence vs. absence of a particular case category in their frame features $(\underline{\text { show }}=+[\quad O+D+A]$ vs. see $=+[$ $O+D]$ Whereas see and look will be synonyms which contrast in the substitution of one case category for another in their respective frame features $\left(\underline{\text { see }}=+[\ldots+D]\right.$ vs. $\underline{\text { look }}=+[[\ldots+A]) .^{1}$

3.53 An analysis on basically similar lines (although without the specific case grammar framework) is involved in Lyons's proposals to treat kill and die as 'alternative, syntactically-conditioned, phonological realizations of the 'same' verb' ${ }^{1}$, or, more specifically, to treat kill as 'the lexicalized' two-place causative form of die', and likewise French montrex as 'the 'lexicalized' three-place causative of voir' ${ }^{2}$. Lyons suggests that the deep-structure descriptions of sentences will employ such labels as ' + ag' (= Agentive) and ' + caus' ( = Causative), and that the lexicon should contain e.g. such information under soft as will permit 'soft: + caus' to be realized as soften ${ }^{3}$.

1. Fillmore $1968 \mathrm{pp} \cdot 30-31$.

1. Lyons $1968 \$ 8.2 .4$.

2. Iyons $1968 \$ 8.2 .14$.

3. Lyons $1968 \oint 8.3 .6$. 
3.54 Fillmore's proposals amount in effect to modifying the scope of the concept 'synonymy' in a way which may be quite simply related to earlier proposals ${ }^{l}$ in generative grammar as follows (ignoring possible differences of opinion about the interpretation of particular examples). Under the definition of synonymy whereby

(D5) C and $\mathrm{C}^{\prime}$ are fully synonymous with respect to PM and PM' if and only if the set of readings associated with the node labeled ' $\mathrm{C}$ ' in PII and the set of readings associated with the node labeled 'C'' in PM' are identical; PM may equal $\mathrm{PM}^{\prime}$ ? $^{2}$

1. e.g. Katz \& Fodor 1963, Katz \& Postal 1964.

2. Katz \& Postal 1964 p. 27. 
it emerges that in the case of constituents consisting of single items listed in the lexicon, two such items must have identical lexical entries in order to qualify as full synonyms. For only on that condition will the readings in question, i.e. the paths comprising a complete sequence of symbols for syntactic marker(s), semantic marker(s), distinguisher (if any) and selection restriction(s), be identical.

Let us now introduce the term 's-reading' to refer to that section of a path in a lexical entry excluding syntactic markers and selection restrictions, i.e. that part of the path comprising only a subsequence of semantic narker(s) and distinguisher if any.

We may now define 'nuclear equivalents' as lexical items which do not differ in respect of their S-readings.

The suggestions made by Fillmore concerning the relation between the pairs like and please, show and see etc. amount to extending the term 'synonymy' to cover cases of nuclear equivalence in instances such as these, together with a concomitant proposal about the deep-structure syntactic characterization of lexical items by reference to cases, this new method of characterisation allowing us to restrict the information required in the s-reading in various ways.

3.55 The question may now be put: What is there to choose between a concept of synonymy which admits nuclear equivalence as a sufficient condition of the synonymity of lexical items (in at least some cases) and one which does not?'

What is ultimately at issue here is the validity of rival views of the boundary between semantics and grammar. In order to count different 
lexical items as synonymous, we must be able to show that any apparent semantic difference between them is in fact 'merely grammatical' and thus can plausibly be accounted for at the grammatical level, leaving the items in question to be characterized as identical at the semantic level.

Fillmore is open to the criticism of failure to provide this justification, and the criticism is not merely that he has omitted to do so, but that his position affords no possible basis for doing so. It is true that Fillmore has shown how to describe certain similarities and differences between words by employing a grammatical terminology ('Agentive', 'Dative', 'subject selection' etc.); but it would be naive to suppose that it followed from the correctness and consistency of such descriptions that the facts described were grammatical facts.

The reason why e.g. like and please cannot be regarded as synonyms which happen to differ in 'subject selection' is the same as the reason why king and queen cannot be regarded as synonyms which happen to differ in 'gender'; namely, that no amount of grammatical description or re-description will reduce the semantic difference. Particular grammatical and semantic facts may be closely interrelated: that does not mean that there is a class of facts with regard to which it is an arbitrary choice, or one of convenience, whether we say they are part of the speaker's grammatical knowledge, or part of his semantic knowledge. And even if there were such a 'borderline' class, the facts about like and please have no stronger claim to be put in it than the facts about king and queen. It is, for example, quite clearly a grammatical fact 
about king and queen that either can be substituted for the other salva grammaticalitate in the sentence frame

The - - Iived in a palace.

To call it also a grammatical fact that the substitution cannot be effected salva significatione would make nonsense of any distinction between grammatical and semantic knowledge. (It would simply leave us to find new terms for a distinction between two essentially different types of 'grammatical' knowledge.)

Similarly, it is a grammatical fact about like and please that either can be substituted for the other salva grammaticalitate in the sentence frame

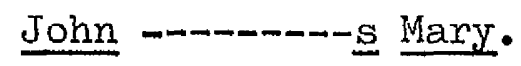

Equally, it is a semantic fact that the substitution cannot be effected salva significatione. But to say this is to say that there is a semantic difference between them. And to say that there is a semantic difference between two expressions is to deny their synonymity. 3.56 If we hold that the boundary between grammar and semantics is relative to the form of Iinguistic analysis adopted, there is nothing to prevent the construction of an account of English according to which

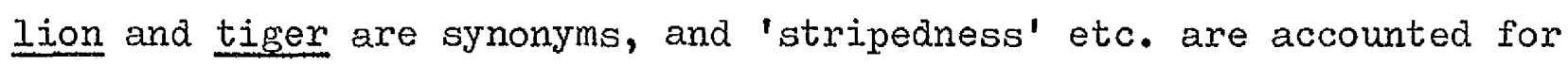
in the grammar as syntactic features. (This absurdity is an absurdity only because no plausible grammar of English is likely to operate in that way, not because no grammar of English could operate in that way.) The alternative is to hold, as has been maintained here, that the difference between grammatical and semantic knowledge is given in advance of linguistic 
analysis. Theories of synonymy based on the former assumption would have no requirements amenable to general discussion, and consequently fall outside the scope of the present study. 
4. Synonymy and semantic analysis. 
4.0 In semantic analysis questions of synonymy arise in connexion with establishing the general (i.e. Ianguage-neutral) conditions governing criteria for relational characterizations of a certain kind in semantic descriptions. A semantic description is here taken to be an account of the semantic knowledge shared by the participants in communication-situations. In the case of a natural language, we postulate semantic knowledge in order to account for certain features of communication between language-users. In the case of a constructed language, the specification of semantic knowledge serves to delimit the possibilities of communication between language-users. In either case, there must be procedures for determining whether given expressions are in fact used in accordance with given semantic characterizations. These are the procedures which, in the case of a natural language, would be appealed to in order to support a correct characterization or to refute an erroneous one.

4.01 There are two types of semantic characterization we are particularly concerned with, and they may be represented as follows:

$$
\begin{array}{ll}
\text { l. } & \underline{a}=\underline{b} ' \\
\rho^{2} . & \text { 'a } \neq \underline{b} ' .
\end{array}
$$


A characterization of the former type tells us that expression a and expression $\underline{b}$ do not differ semantically, while a characterization of the latter type tells us that expression a differs semantically ( in some unspecified respect) from expression b. It is proposed to explicate the notion of a symonymity statement for purposes of semantic analysis - i.e. to answer the question "what is it to say of two expressions of $\underline{L}$ that they are (or are not) synonymous?" - by reference to characterizations of the above types. We shall examine how such a statement might be justified for any pair of expressions $\underline{a}$ and $\underline{b}$ in a language $\underline{\underline{L}}$. To do this, it will be necessary to consider how $\frac{\rho}{t}$-characterizations are related to characterizations of a different kind.

4.02 It is clear that a semantic description of $\underline{L}$ which provided only semantic charactexizations of type ' $\mathrm{fl}$ ' and type ' $\mathrm{g}^{2}$ ' would be incomplete in the obvious sense that, while telling us whether any expression of $\mathrm{L}$ did or did not differ semantically from any other expression of $\underline{L}$, it would fail to tell us in what way any two semantically differing expressions differed. It would thus fall short of an adequate account of a competent speaker's sementic knowledge of those expressions. For example, a semantic description of English which includes the following $\rho^{2}$ characterization

$$
\text { 'rhinoceros } \frac{1}{r} \text { dibatag' }
$$

tells us that the words rhinoceros and dibatag differ semantically. 
But in order to discover how they differ we should need to have available semantic characterizations of a different type from ' for example

'rhinoceros: quadruped with horned nose.......'

'dibatag: long-necked antelope..........'.

Characterizations like these will be termed ' $\sigma$ - characterizations' or 'substantive semantic characterizations' and represented as

$$
\text { 'a: } \alpha \ldots \ldots \ldots \text { ' }
$$

to distinguish them from the 'relational semantic characterizations' of type ' $\mathrm{g}^{1}$ ' and type ' ${ }^{2} 2$ '. These typographical conventions correspond to the fact that 'a' ', ' $\underline{b}$ ' etc. are expressions of the object-language (or language under description), while ' $\alpha$ ', ' $\beta$ ' etc. are expressions of the metalanguage (or language of description).

We may distinguish between a stronger and a weaker condition to be imposed on semantic characterizations. In the case of $\sigma$-characterizations, the weaker condition is met if the metalinguistic description states a meaning for the given expression, but without indicating the semantic structure of the expression, i.e. without analysing the expression into its meaning-beaxing elements, or stating how a composite meaning for the whole is provided by the arrangement of these elements. For example, the characterization 'sour apple' for an expression $\underline{e}$ does not in itself indicate $\mathrm{e} . \mathrm{g}$. whether or not there is one part of $e$ which means 'sour' and another part of $\underline{e}$ which means 
'apple', and another part, or leature of arrangement, of $\underline{e}$ which combines these two meanings in the way indicated by the metalinguistic expression 'sour apple'. The stronger condition for $\sigma$-characterizations is that they should provide precisely such an analysis. We may term characterizations which meet only the weaker condition 'synthetic characterizations', and those which meet the stronger 'analytic characterizations!. In keeping with the distinction earlier drawn (3.27, 3.4) between synthetic and analytic synonymy, we may likewise distinguish between synthetic and analytic p-characterizations, the former indicating only whether two expressions do or do not differ semantically as wholes, and the latter whether or not they differ semantically in respect of their internal structure. For notational convenience, we may distinguigh when necessary by prefixing 'S' in the case of synthetic, and 'A $\underline{\text { ' }}$ in the case of analytic characterizations (So, $\left.\underline{\Delta} \sigma, \underline{S_{\rho}} 1, \underline{\Delta \rho} 1, \underline{S_{\rho}} 2, \underline{A_{\rho}} 2\right)$.

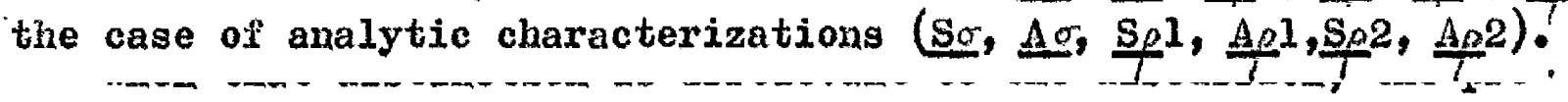

fixed letter may be omitted.

In this way it is possible to treat a semantic description

1. Txplicit proposals for systematizing semantic descriptions are discussed in Katz \& Fodor 1963, Katz \& Postal 1964, Weinreich 1966, Katz 1967, and elsewhere. Such systematizations are not our present concern. Since the issues to be discussed in the present chapter arise independently of any specific proposals as to the best means of arranging the information in a semantic description, it will be preferable to continue to treat senantic characterizations in the abstract way exemplified above. For our interest here is in the content of a characterization, not in how that content ought to be represented, broken down, and related to other characterizations by means of devices such as projection rules, semantic markers etc. Furthermore, confinexnent to a systematization of the kind currently accepted in work on the semantic component of a generative graimar is unacceptable, since that model embodies presupposition of the semantic determinacy of expressions, a position which is not well established (see below, and Bolinger $1965 \mathrm{p.571}$ ). 
as a store of information organized so as to produce when required $\cong$-characterizations and $\rho$-characterizations; that is, for any given expression the description will provide a sequence of metalinguistic symbols (its $\sigma$-characterization) fornulating the competent spealker's semantic knowledge of that expression, and for any given pair of expressions the description provides a metalinguistic symbolization ( $f$-characterization) expressing their semantic equivalence or non-equivalence. Such a description, if exhaustive, gives a complete account of the semantic lmowledge of a speaker of $\underline{L}$ in the sense of including the information that would be given by a complete inventory of the expressions of $I$ with their substantive semantic characterizations. It will tell us not merely that expressions of $\underline{\underline{L}}$ differ semantically, but in detail how they differ semantically. Although a complete inventory, i.e. list, cannot be drawn up if $\underline{\mathrm{L}}$ is a natural language, because of the indefinitely large number of sentences in any natural language, the information available will nonetheless enable us to find the appropriate substantive semantic characterization for any desired sentence. By the same tolsen, a complete list of synonymous expressions cannot be drawn up: the most we can hope for if $\underline{L}$ is a natural language is to be able to determine for any desired pair of expressions whether or not their substantive semantic characterizations are the same. 
This completes our account of the mininum conceptual apparatus required to tackle synonymy as a problem of semantic description. The next step is an inquiry into the justification of $f$-characterizations.

4.1 We may approach the question of the relationship betreen relational characterizations (e -characterizations) and substantive chaxacterizations ( $\mathscr{\sigma}$-characterizations) by supposing iirst of all that we have available a completed semantic description of L, and postulating that it is organized in such a way that the $f$-characterization for $\underline{a}$ and $\underline{b}$ expresses the identity or nonidentity of whatever $\underline{\sigma}$-characterizations are assigned to $\underline{a}$ and $\underline{b}$. On this assumption, relational characterizations of type ' $\mathrm{gl}^{1}$ ' are abbreviated statements to the effect that $\underline{a}$ and $\underline{b}$ have the same Q -characterization, while relational characterizations of type ' $\mathrm{g}^{2}$ are abbreviated statements to the effect that a and $\underline{b}$ do not have the same $q$-characterization. We may term a semantic description of $\Phi$ which gives both relational and substantive characterizations an 'internally consistent' description if for any $\underline{a}$ and any $\underline{b}$ a $\phi$-characterization ' $\underline{a}=\underline{b}$ ' appears if and only

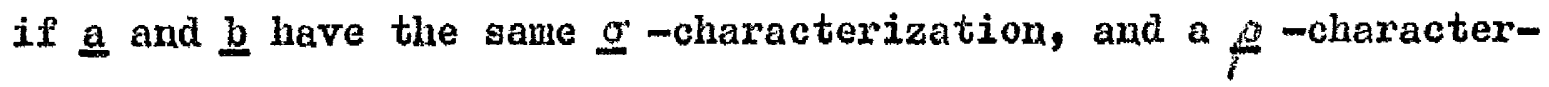
ization 'a $\frac{1}{f} \underline{b}$ ' appears if and only if $\underline{a}$ and $\underline{b}$ do not have the same $\sigma$-characterization. In the case of synthetic of -characterizations there will be a corresponding synthetic $f$-characterization 
(of type $\frac{\mathrm{Sol}}{i}$ or $\frac{\mathrm{S} \rho}{\mathrm{T}} \mathrm{2}$ ), while in the case of analytic 0 -characteriz-. ations there will be corresponding analytic $\rho^{\text {-characterization }}$ (of type $\frac{A_{\rho} 1}{f}$ or $\frac{A_{g} 2}{f}$ ).

4.11 A criterion has now been formulated by which the internal consistency of semantic descriptions may be judged. Obviously, however, a particular semantic description of $\underline{L}$ might easily pass the criterion of internal consistency, yet be wrong in the sense of giving an erroneous account of the semantic facts. For examples, a semantic description of Inglish which contained the $\rho$-characterization

$$
\text { 'rhinoceros }=\underline{\text { dibatag' }}
$$

and the $\sigma$-characterizations

'xhinoceros: quadruped with horned nose....'

and 'dibatag: quadruped with horned nose.....' would be (in this respect) an internally consistent description, but it would be wrong in view of the fact (granted that it is a fact) that for speakers of English the words rhinoceros and dibatag are not senantically identical, and specifically in view of the fact that 'quadruped with horned nose...' (granted the usual sense of these words in English, here talsen to be the metalanguage) is not a correct semantic characterization of dibatag.

It follows that we cannot adequately characterize that semantic lnowledge which is lmowledge of synonymy in terms of the 
structure of a description of $\underline{\underline{L}}$, since the strongest guarantee of synouymy this account gives is one of internal consistency of the semantic description of $\underline{L}$. But because it is possible to construct an internally consistent description which could be wroag about instances of synonynity, there would be no way of deciding which of two different but internally consistent semantic descriptions of $\underline{L}$ correctly represented the lnowledge of symonyuy of speakers of $\underline{I}$.

The difference between two rival semantic descriptions need not involve gross incompatibilities (as between a descxiption of Dnglish which included the $f$-characterization 'rhinoceros $=$ dibatag' and a description of Ihalish which included the $f$-characterization 'rhinoceros $\neq$ dibatag' $)$. It might be the case that description ' $\Lambda$ ' dravs more or different semantic distinctions as compared vith description ' $B$ ', with the result that expressions which are characterized as semantically equivalent under one description are characterized as showing a slight semantic difference under the other ${ }^{1}$. Thus we should have two rathex different accounts of the speakers' knowledge of synonyny; but again the question which account was to be preferred could not be settled by reference to the intermal structure of the descriptions concerned.

1. This situation is found in dictionaries which purport to be dictionaries of the same natural language. One dictionary draws a distinction between two words which another dictionary glosses identically. 
4.12 The reason why proposals in semantics which afford no basis for external criteria of synonyuy (i.e. criteria which go outside the structure of the semantic description) lack the means of deciding questions about the correctness of $p$-charactizations is that they leave unclear which facets of the speaker's semantic lnowledge are to be understood as corresponding to the concept 'synonymy'. For example, in 'the structure of a semantic theory' Katz \& Fodor spealk of 'the ability to interpret sentences', and distinguish various aspects of this ability:

'The spealser's exercise of this ability....provides empirical data for the construction of a semantic theory, just as the construction of a gramur draws upon empirical data supplied by the exercise of the speakex's ability to distinguish well-formed sentences from ungramatical strings, to recognize syntactic ambiguity, and to appreciate relations between sentence types. A semantic theory describes and explains the interpretative ability of speakers: by accounting for their performance in determining the number and content of the readings of a sentence; by detecting semantic anomalies; by deciding upon paraphrase relations between sentences; and by marlsing 
every other semantic property or relation that plays a role in this ability.' 1

This formulation could perhaps serve as a model for drafting a preliminary statement about that aspect of the speaker's semantic lmowledge which corresponds to the concept 'synomyny'. If for: 'ability to interpret sentences' one substitutes 'ability to interpret expressions', then we could suppose that the speaker has the ability to place every pair of different expressions in the language into one or other of two classes, according to whether members of a given pair do or do not receive the same interpretation. Innowledge of synonyuy would thus correspond to that part of interpretative ability which informs the speakex's judgments of interpretational 'sameness' and 'difference'.

But this talkes us only as far as the notion of 'f -characterization' already discussed. For talls of the speaker's ability to put pairs of expressions into classes canmot be taken as indicating a type of informant test criterial for determining instances of synonymity. Until further progxess can be made towards stating what typical skills flow from knowledge of synonymy, no wore has been done than to draw a tentative

1. Katz \& Fodor 1963 p. 486. 
and quite general distinction within a certain area of linguistic knowledge. (It is as if one had said, for example, that it is to be supposed that part of having normal vision is being able to detect similarities and differences of colour: doubtless true, but no more than a point of departure and, as the statement of an explicandum, a vague one at that.)

The clarification supplied in 'The structure of a semantic theory' is hardly satisfactory. A distinction appears to be dram between two types of case where a language might have expressions differing in foxm but identical, in meaning, the term 'synonymy' being restricted to expressions which constitute individual lexical items in the dictionary, and the term 'paraphrase' being applied to expressions which constitute sentences of the language. No account is given of conmunicational skills which correspond to knowledge of synonyms; but there is an account of 'paraphrasing skill'. The coumunication-situation imagined is that of speakers of Inglish receiving an anonywous letter containing only the Bnglish sentence 'S'. Their ability to intexpret ' $\underline{ }$ ' is contrasted with that of 'pexsons who do not speak Inglish, but are equipped with a completely adequate gramar of English'. 'Paraphrasing slrill' is listed among the abilities of the speakers of Inglish, and described in the following terms: 'Tinally, whatever sentence the anonymous lettex contains, as a rule, speakere of Inglish can easily decide what sentences are paraphrases of it and what 
are not in the sense that they can answer such questions as: what does the letter say? does the letter say such-and-such? how can what the letter says be replurased? This facet of the speaker's ability camnot be referred to his wastery of grawnar either, for the group who are equipped with a grammar but who do not speak Tuglish will be unable to tell whether or not a sentence is a paraphrạse of $\mathrm{S}$. The reasons are simply that there need be no definite grammatical relation between a sentence and its paraphrases; e.g. "Two chairs are in the room" and "There are at least two things in the room and each is a chaix;" and that where a definite grammatical relation obtains between a pair of sentences, neither need be a paraphrase of the other, e.g. "The ball was hit by the man" and "The ball was hit," "Ihe man hit the ball," and "The man did not hit the ball." Thus, still another facet of the speaker's semantic ability which must fall within the dowain of a sewantic theory is his parapỉnà sing skill. 2 
This account invites, if it does not involve, a serious confusion between speech acts (e.g. those comprising a letter) and sentences. 'What does the letter say?' is a question about speech acts, and so are the other questions put to the two imaginary groups of 'inforwants'. It thus appears that the Katz-Fodor account of paraphrasing slcill is based on a latent theory of speech acts; but this theory is never made explicit, still less justified. Insofar as one can reconstruct it from the somewhat scanty remarlss in 'The structure of a semantic theory', it appears to involve at least the following thesis:

(A11?) sentences in $\mathrm{L}$ can be grouped into pairs (e.g. 's1' and 'S2') or larger sets ('S1', 'S2', ' $\$ 3$ '....) of paraphrases, between which certain (so far unspecified) semantic relations hold. A speech act which is a question about the purport of another (any other?) speech act involving the utterance of ' $\mathrm{Sx}$ ' can be correctly (and fully?) answered if (and only if?) the answerer knows whether some other sentence 'Sy' is a paraphrase of ' $\mathrm{Sx}$ '.

But if this partial reconstruction of the speech act theory underlying 'The structure of a semantic theory' is in essentials correct, it becomes evident that the only account given 
of 'paraphrasing sirill' is a circular one. That is to say, 'paraphrasing skill' is presented as (paxt of) the knowledge which enables the speaker to pexiorm successifully certain speech acts, the speech acts in question being those which manifest a lnowledge of paraphrases. This circularity is furthex evident in Katz \& Fodor's comments on projection rules: e.g. it is asserbed that the semantic interpretation assigned by the projection rules must 'account... for the speakex's ability to understand semtences by, inter alia, suitably relating 'sentences speakexs know to be paraphrases of each othex ${ }^{3}$. In other words, the rules must account for paraphrasing skill by wark-ing paraphrases as paraphrases.

This leaves an explanatory gap in 'The structure of a semantic theory', a gap which is not - and could not be - Rilled by occasional exemplification: i.e. it is of no avail to an that e.g. Where axe at least two things in the room and each ig a chaix is a paxaphrase of Two chairs axe in the room. Wor this is merely to agree to call a cextain pair of sentences 'paraphrases', $i . e$. we here explain 'paraphrasing skill' as knowledge that e.g. a and $\underline{b}$ 'axe paraphrases'.

3. Katz \& Fodor 1963 pp.494-4. 
But what one wants to know is not what to call such sentences,

but just what it means to call them 'paraphrases' 4.

There might be two possible explanations of this lacuna

in 'The structure of a semantic theory'. One would be that

'paraphrase' is not defined because there is already an est-

ablished and generally accepted theory of paraphrases, too

4. A sinilar objection may be brought againgt the use of the term 'paraphrase' by Southworth, who proposes 'that we consider as basic linguistic data the information that cextain parts of a corpus are paraphrases of each other' (Southworth $1967 \mathrm{p} .345$ ). In Southworth's case the objection is particularly crucial, since 'paraphrase' is a key term. Southworth's claim is that paraphrase is one of the two 'intex-sentence relations' to which all others can be reduced. But it is not at all clear what Southworth means when he calls certain sentences of Jnglish 'paraphrases'. I.g. The committee rejected the proposal unanimously is said to have as "paraphrases ' both The committee's rejection of the proposal was unaximous and Each of the committee members rejected the proposa1. But it seems at least contentious that the truth conditions for these three sentences are identical, and thus in doubt whether they have the same meaning. Southworth's comment that to call two sentences 'paraphrases' is not the same as saying that they 'mean the same' is haxdly illuminating, and apart from examples the only explanation of his claim is, unfortunately, a definition which relies on a prior notion (that of 'juplication') which stands in just as much need of clarification. 
well lonown to need explicit recapitulation. But this cannot be the explanation because in 1963, when Katz \& Fodor's paper was first published, there was no such theory. The other explanation would be the 'we-al1-know-(roughly)-what-is-meantby-'x'l explanation. But this is an explanation which will not do at all here. Insofar as we do lnow (roughly) what is meant by lKatz \& Podox's 'paraphrase', it is because we have sead other (equally vague) gramatical and philosophical writings where that texm is employed, not because we lnow as a mattex of experience as language-users that thexe 'are paraphrases', that paraphrases 'really exist'. (The case is analagous to that of 'sense datum'. The term 'sense datum' gets is sense from its (somemhat controversial) role in discussions of the philosoply of perception, not from oux obsexvations of people (including ourselves) engaged in pexceiving. Accordingly, it would be quite question-begging to impose as an empirical constraint on a theory of perception that it account correctly for sensemdata.) $0 f$ course, doubtless spealrers do, in particular situations, react to the uttexance of different sentences in ways which axe in cextain respects similax. But in oxdex to clarify the concept 'paraphrase', what needs to be made cleax is which of these similarities, or which aspects of these similarities, are under discussion when the texm 'paraphrase' is used. 
The question then arises: what is the point of imposing upon a semantic theory - or upon any theory, for that matter an empirical condition of adequacy ' $\mathrm{K}$ ' (in this case ' $\mathrm{K}$ ' is 'coxrect account of paraphrasing slsili') pxior to a definition of the texms used in stating 'IS'?

The answer is: none. And the obvious explanation for the appearance of such a condition in 'The structure of a semantic theory' is simply that the alleged semantic skills have been invented in advance to pxovide explanatory functions suited to the capacity of the model which its authors are about to propose for the semantic component of a linguistic description.

Ifo say this, it should be noted, is not to deny the utility of the model: it is simply to advocate dropping the pretence that 'IS' states an empirical constraint.

4.13 One further genexal point should be made concerning the correlation of descriptions to describienda. One might agree with someone who maintained that an ingortant requirement for road-maps was that B-roads should be marked as B-roads. Agreement with such a pexson is not entirely vacuous, inasmuch 
as there might be others who maintained the (surprising) thesis that B-roads should be marked as $A$-roads, or others again who maintained that B-roads should never be marked at all. Thus agreeing with Katz \& Fodor that paraphrases should be marked as paraphrases is to cast a vote against e.g. such proposals as that paraphrases should be marked as semantically anomalous, or that paraphrases should not be indicated at all. But the theorist of map-malsing may have a critic who maintains that, since a map purports to provide correct information about roads, unless there is an agreed definition of what counts as a B-road it may be misleading for a wap to distinguish between B-roads and others. The same critic might also point out that, in the absence of such a definition, it is difficult to know exactly what insistence on the principle of marking B-roads as B-roads amounts to. To such a critic our theorist of map-making wight perhaps reply: "Bverybody knows what a B-road is. In any case, if there is someone who doesn't, then he should not look to me to supply a definition, because it is not my job." (One would then form an appropriate view of his claim to be a theorist of map-malking.) But it would be even sillier of him to reply: "0h, but a map constructed according to my principles tells you which the B-roads are. The map itself supplieg the definition." If our theorist replied in this latter sense, he would have missed the point of the question, and have given a 
circular answer into the bargain. Anyone who supposed that 'The structure of a semantic theory' offered a means of defining 'paraphrase' for any language described according to the principles there advocated would be involved in an exactly analogous circularity.

4.2 The conclusion argued for thus far has been the purely negative one that an adequate account of lnowledge of symonymy cannot be provided in texms of the structure of the semantic description itself. Consideration will now be given to a moxe positive specification of the xequirements, i.e. we shall inquire what constraints external to the structure of the semantic description of $\underline{\mathrm{L}}$ affect setting up $p$-characterizations. We shall first consider certain constraints which follow from the basic reasons for the explanatory postulation of semantic lnnowledge. Hexe there arises the question of how linguistic lruowledge is connected with pragmatic judgments arising out of communication (e.g. judging whether what someone says is true, what the coxrect answex to someone's question is, etc.), such judgments presupposing the prior coxrect interpretation of words used in speech acts. We shall be particularly concexned with the comaexions between the meanings of sentences and the possibility of usiag sentences to make brue ox false statements. 
4.21 Semantic knowledge is postulated - on the view we are here taking - in order to account for the possibility of communicational exchanges, including the exchange of infoxmation about extralinguistic lacts. But, fox epistemological reasons, acquiring lonowledge of the truth of $\mathrm{p}$ cannot be equated with undergtanding a verbal expression in which the statement $p$ is foxmulated. Otherwise, to cite Arege's example ${ }^{3}$, it could have been lnown without the help of astronomical observation that the sentence Mhe Morning Stax is (the same as) the grening Stax expressed a true statement. By the same tolsen, we are led to distiaguish meaning from reference ${ }^{2}$. For if an identity statement expressed by a sertence of the form 'a is $b_{\mathrm{g}}$ ' is true, then it must be the case that one and the same item $X$ is referred to both by the expression a and by the expression $b$ But if to undexstand the expression a were no more and no less than to know that $X$ is referred to by a $a_{\text {g }}$ and to understand the expression b were likewise to lnow that $\mathrm{X}$ is referred to by $\underline{\mathrm{b}}$, then anyone who understood the sentence ' $\mathrm{g}$ is $\mathrm{g}$ ' would also know the truth of the identity statemeat.

1. Frege 1892.

2. In Frege's temuinology, Sinn from Bedeutung. But the re axe reasons for questioning the usual translation of Frege's Bedeutung by reference (Tugendhat 1970). 
4.22 The argument from identity statements enables us to make some progress towards identifying the slrills which flow firom lnowledge of symonymy, for it makes apparent one constraint upon setting up $p$-charactexizations, if the lattex are to serve the purpose of representing knowledge of symonyay.

Considex a proposed semantic description Sd of a language La in which a and I are proper names having the same beaxex I.

Let us suppose that $\mathrm{Sd}$ gives the following $\sigma$ - characterizations:

'a: proper name of $\Sigma$ ?

'b: propex name of $\Sigma ;$

(where 'I' is an individual constant in the metalanguage).

It follows that if Sd is an internally consistent description by the eriterion previously proposed, Sd gives also the e -charactexization:

$$
t_{\mathrm{a}}=\underline{b^{\prime}} \cdot
$$

But here the $\sigma$ - characterizations represent the fact that

1. T.t is sometimes held that proper names 'have no meaning' (for a cxiticism of this doctrine, see 5 frensen $1.963 \mathrm{Ch} .4$ ), and it might therefore be questioned whether proper names fall within the scope of a theory of symonymy. The supposition underlying the present discussion is that a semantic description assigns a $\sigma$-characterization to any expressions whose interchange systematically affects the contient of speech acts. Thus if English spealsers use e.g. Is this the road to Pdinburgh? to ask a different question from Is this the road to loondop?, and likewise I live in Edinburgh to make a different statement fron I live in Iondon, etc. then the propex names pdinburgh London are assigned $\sigma$-characterizations in a semaxtic description of English. The view that proper names have meanings is argued for in Frege 1892. Cf, also Searle 1958. 
speakers of $\underline{\underline{a}}$ know the identity of reference of $\underline{a}$ and $\underline{b}$, which, if we accept the argument from identity statements, cannot be equated with knowing that $\mathrm{a}$ and $\mathrm{b}$ have the same meaning. Thus if we wish oux $f$-characterizations to indicate synonymities, we are led to stipulate a prohibition on setting up relational characterizations of type ' $\rho \mathrm{l}$ ' in any instance where the relevant on-characterizations merely supply an individual constant identifying the bearer of a name.

It should be noted that this prohibition also sets a condition on the completeness of semantic descriptions. This is evident when we consider the result of applying the prohibition to Sd. Let us call the semantic description so modified 'Sdi'. In $\underline{\text { Sdi }}$ we are debarxed from setting up the $\rho$-characterization $' \underline{a}=\mathrm{b}$ '. Should we, then, set up the ' $p 2$ ' characterization 'a $f b^{\prime}$ ' But this is not allowed either, for such characterizations are - so far - set up if and only if the relevant g-characterizations differ, and in this case they do not differ. Thus we have a pair of expressions of the same semantic type (proper names), for which Sdi fails to provide any $f$-characterization at all. It follows that Sdi cannot be a complete description of La.

An exactly parallel case can be constructed substituting for the proper names $\underline{a}$ and $\underline{b}$ in $\underline{L}$ a two referring expressions which are not proper names. In this way the argument is generalized from 
proper names to referring expressions of all kinds, and an exactly parallel prohibition on setting up $\rho$-characterizations rast be extended to all referring expressions. Thus if the $\sigma$-characterizations for referring expressions in La merely supply individual constants of the metalanguage identifying the referents of those expressions, no corresponding $f$-characterizations can be set up, and it wust be concluded that the semantic description is incomplete. Such a conclusion is obviously correct, the missing element in the semantic description being a representation of the lnowledge which might enable users of La to understand sentences formalating identity statements without ipso facto lnowing the truth or falsity of the statements in question.

4.23 Frege's argunent from identity statements may be developed further to throw some light on synonymy of referring expressions. For if it is accepted that astronomical observation is required to determine whether or not a statement formulated as Whe Morning Star is the prening Stax is true, it is thereby implied that semantic knowledge is inadequate to settle that question, i.e. that the truth in question is not an analytic truth, and the sentence in question not an analytic sentence.

1. We may define an analytic truth as one belonging to a certain conceptual system within which its denial is self-contradictory. It is thus, relative to the language in which it is expressed, a truth 'grounded in meanings independently of matters of fact! (quine $1961 \mathrm{p.20}$ ). Coxrespondingly, an 'analytic sentence' may be defined as one expressing an analytic truth. 
But if this is so, however the meanings of the expressions The Morning Star and the Mvening Star be defined, it is at least clear that these expressions are not synonymous referring expressions. For if they were, then the truth of the idemtity statement would be assured without reliance on astronomers, since to question it would be to question the truth of a tautology which might be alternatively formulated as The Morning Star is the Morning Star.

This conclusion assumes: (i) that we are not dealing with 'refexentially ambiguous' expressions ${ }^{2}$, (ii) that we are concerned with a conceptual system in which the denial that $X$ is $X$ is self-contradictory, and (iii) that the copula sentence is 'semantically endocentric' with reppect to the referring expressions occurring in it, i.e. that this is a case where the meaning of a sentence is a function of the terms

2. Sfomeone who says John is not as tall as John does not coatradict himself in the way he would have done by saying John is not as tall as himself: in the former case, we would normaliy suppose that Jolm is referentially ambiguous, i.e. there are two different individuals involved who happen to have the same name. Referential ambiguity also arises with deictic expressions: e.g. saying This is Luther's translation of the New Testament, and this is the American Standard Version does not comnit anyone to the proposition that one and the same text is simultaneously Juther's translation and the American Standard Version as well. 
which occur in the sentence. ${ }^{3}$

We may for purposes of the present discussion define

'semantic endocentricity' as follows. Any expression is semantically endocentric with respect to its parts if and. only if the meaning assigned to it is exclusively determined by the meanings of the parts and of their combination."

3. Hates 1950 p.120. The sense of 'function' here is that borrowed from mathematics, the constituent terms and the manner of their combination being regarded as the semantic variables.

4. An expression which is not semantically endocentric is 'semantically exocentric'. Thus, for example, the Inglish phrase red roof is semantically endocentric with respect to the parts red and roof if the meaning of the phrase is uniquely determined by the meanings assigned to red('ruber'), roof ('tectum'), and the adjective + noun combination ('y that is $x$ '). Whereas the phrase white elephant is semantically exocentric if either it has no meaning deternined by the meanings assigned to white ('albus'), elephant ('elephas'), and the adjective + noun combination ('y that is $x^{\prime}$ ), or, as well as this meaning, has independently some other meaning ('unwanted gift'). (The question may arise as to why, in such a case, we choose not to give a semantic analysis according to which in white elephant the adjective white way mean 'unwanted' and the noun elephant way mean 'gift'. One anstrer might be that if neither white nor elephant occur with the meanings 'unwanted' and 'gift' respectively outside the one phrase white elephant, then the meaning 'unwanted gift' is peculiar to that phrase and cannot appropiriatedy be considered the product of the independent meanings of white and elephant. If, on the other hand, white were found in other cases meaning 'unwanted'and elephant in others meaning 'gift', the justification for regarding white elephant as semantically exocentric would lapse. This is not an entirely satisfactory answer, however, for there might be reasons, even were there other cases where white meant 'unwanted' and elephant meant 'gift', for regarding white elephant as semantically exocentric. One such reason would be if neither white gift nor unwanted elephant meant' 'unwanted gift'. 
On the basis of assumptions (i) - (iii) we may generalize as follows. For any language $\underline{L}$, let 'referentially unawbiguous identity sentence' (or 'I-sentence') be defined as a two-place copula sentence $(1 \ldots$ is...') in which each place must be filled by a referentially unambiguous referring expression of $\underline{L}$, such that the identity statement expressed asserts the referent of the first-place expression to be (or, in the case of a negative I-sentence, not to be) identical with the referent of the second place expression. Now if the statements made in $I$ belong to a conceptual system in which the denial that $X$ is $X$ is self-contradictory, a subclass of I-sentences of I will be analytic sentences. Any affixmative I-sentence vill be analytic which conforms to the following rule:

$$
\begin{aligned}
& \text { '. : for any first-place referring expression a } \\
& \text { which has } X \text { as its referent, the second-place } \\
& \text { referring expression does not diffex in } \\
& \text { meaning from a. }
\end{aligned}
$$

For the supposition that the referring expressions in the same affirmative I-sentence might have different referents but not differ in meaning is incoherent, i.e. involves supposing that either 'a is a' might be ialse or 'a is b' might be ialse even when a and $\underline{b}$ ex hypothesi do not differ in meaning, or both. But, given (i) and (ii), 'a is a'cannot be false, and if 'a is a' camnot be false, 'a is b' cannot be 
false either, since it differs from 'a is a' only in the substitution of an expression which does not differ in meaning from a, and thus, given (iii), 'a is a' and 'a is $\underline{b}$ ' do not differ in meaning.

Thus at least there is a clear explication available of the notion 'synonyuy of referring expressions in I-sentences': namely, the notion of the relationship between referring expressions which diffex in form but comply with $\underline{x}^{\prime}$. It follows that if $\underline{L}$ includes I-sentences, the competent speaker's knowledge of L-synonymy may be partially explicated in these terms, and we may say that lnowledge of synonymy enables the competent speaker, inter alia, to determine which affirmative I-sentences of the form 'a is $\underline{b}$ ' reduce, by substitution of synonyms, to 'a is a '.

Any language which had $\underline{x}^{\prime}$ as a mandatory rule for afíirmative I-sentences would be characterized by the following features:

(1) For any comnunication situation such that $\mathrm{X}$ is the referent of a, any affirmative I-sentence of $\mathbf{I}$ containing a can be used to malse a true identity statement about $x$.

(2) All affirmative I-sentences of $\mathrm{L}$ are analytic provided the referring expressions are not devoid of reference.

It seems clear that natural languages contain I-sentences 
of the type 'a $i$ is $\mathrm{a}^{\prime}$ ', e.g.

(3) The highest mountain in the world is the highest nountain in the world

but equally clear that the generalizations formulated in (1) and (2) are counteringtanced respectively in natural languages by sentences like

(4) Ihe highest mountain in the world is the highest mountain in the British Isles

and (5) The highest mountain in the woxld is the highest mountain in Nepal.

We way thexefore tentatively characterize natural languages as languages in which I-sentences occux but $\underline{x}^{\prime}$ is not mandatory. Thus some progress has been made towards identifying the communicational skills which flow from lnowledge of synonymy in the case of the speaker of a natural lamguage.

4.24 Further progress can be made by involing the vexy powexful argunent offexed by Mates, applicable to axy semantically endocentric sentence of $\mathrm{L}$ which can be used to malse a true-or-false statement. If we change such a gentence solely by replacing one component expression by a syaonymous expression, the resultants sentence must be synonymous with the original sentence, since, by the postulate of semantic endocentricity, the meaning of the whole has not been altered. In any circumstances under which the oxiginal sentence could be used to make a true statement, the 
resultant sentence can likewise be used to make a true statement, and in any circunstances undex which the original sentence could be used to make a false statement, the resultant sentience can liksewise be used to make a false statement. Hence 'synonymous expressions way be intexchanged without affecting the meaning or the truth value of the sentences in which they occux'. 1

The more interesting question then axises whethex interchange of expressions without altering the truth value of: senteaces in which they occur is a sufficient condition of synonymity. On this point, Mates offers anothex axgument, which may be summarized as follows.

In the tautological identity sentence 'a is a', a may be replaced by any expression $\underline{b}$ and the statement remains true, provided 'a is b'is also brue. But if the semantically endocentric sentences of $\mathrm{I}$ which may be used to malke true-orfalse statements include sentences containing a modal operator ('N' = 'necessarily'), a stronger guarantee is requixed; for 'N (a is a $)^{\prime}$ will be txue but 'N (a is $\left.b\right)$ ' may be false. This strongex requirement is that 'a is b' should be not merely true, but an analytic truth. But should the semantically endocentric

1. Mates 1950 p. 210 . 
sentemces of $I$ which may be used to malse true-or-false statements also include sentences for reporting indirect discourse, beliefs etc., even this requirement is not strong enough. For since Jones may believe that 'a is a' without believing that 'a is $\underline{b}$ ', the sentence 'IN (Jones believes that a is a $\underline{a}$ if and only if Jones believes that $\underline{a}$ is $\underline{a}$ )' may be used to make a true statement, but 'N (Jones believes that a is a if and only if Jones believes that $\underline{a}$ is $\underline{b}$ )' a Palse one, even where 'a is $\underline{b}$ ' expresses an analytic truth. The stronger requirement in this case is that 'a is 므' should be not merely a sentence expressing an analytic truth but that a and $\underline{b}$ should be synonymous.

'That nothing short of synonymity will guarantee interchangeability in a language of this type follows from the fact that the truth value of a sentence 'Jones believes that $A$ ' depends not upon the truth value of the constituent $A$ but upon its meaning. If $A$ is replaced by any other expression not having the same meaning, the truth value of 'Jomes believes that $A^{\prime}$ may be changed, which implies that the truth value of ' $N(J o n e s$ believes that $A$ if and only if Jones believes that $A)$ ' will be changed. Consequently if two sentences $\Lambda$ and $B$ are not synonymous, they will not be interchangeable in all sentences of our language. Similax considerations lead to the further conclusion that if any tro sentence constituents $\mathrm{x}$ and I are not synonymous, then they will not be interchangeable in 
the true sentence,

'N(Jones believes that.... .... if and only if Jones believes that.... ....)'., 2

If we accept that natural languages fall within the class of languages to which Mates's argument applies, the question remains whether or not this requirement is, as Linsky ${ }^{3}$ suggest, 'too strong'. For what Linslis calls 'paxadiguatic synonym pairs' (e.g. bachelox and unmarried man) fail to pass Nates's criterion, in view of sentences like

(6) Jones wants to Inow whether a bachelor is an unmarried man

and (7) Jones wants to know whethex a bachelor is a bachelor.

Linsky's exemplification of a 'paradigmatic synonym pair' is unfortunate, since it seems clear that bachelox and unmarried wan are at least not analytically synonywous. But we do not need to rely on paradigmatic counterexanples at all, in view of the following considerations.

In the sentence frame

(8) Jones wantg to lnow whethex a .... is a ..... we can fill the two blanks either by the same form ( $a$ and a)

2. Mates $1950 \mathrm{pp} \cdot 211-212$.

3. Linsliy $1967 \mathrm{p} .55$. 
or by two different forms (a and b). Now regardless of the meanings of a and $\underline{b}$, it is clear that the two sentences so produced can report different things that Jones wants to lnow. But if that is the case, then difference of form must be a suficient condition of difference of meaning for any language which contains sentence frames like (8) and any expressions which may fill the blanlss in such frames. One consequence of this for a language like Hnglish would be that no two nouns or noun phrases are synonymous.

But postulating a difference in meaning between every noun (or noun phrase) and every other noun (or noun phrase) would be not so wuch too drastic a way of accointing for the difference in meaning between sentences about what Jones wants to know as a very puzzling way. For it will be far from clear in many cases exactly what semantic difference could plausibly be introduced into the $g$-characterizatious of pairs of expressions such as to account for their noninterchangeability in frames like (8). N.g. if it happens to be true that

(9) Jones wants to know whether a pomelo is a shaddock but not true that

(10) Jones wants to lowow whether a pomelo is a pomelo then presunably Jones's uncertainty is occasioned by not lnowing the exact relation between the fruit and one or both of the expressions pomelo and shaddock, i.e. Jones is uncextain as to 
whether or not it is the same fruit which is variously called powelo or shaddock, but not uncertain as to whether or not a pomelo is a pomelo. What at least seems clear is that postulating a difference in meaning between pomelo and shaddock is no way of explaining how it comes about that Jones's uacertainty is correctly reported by (9) but not by (10). For that is already explained by the occurence in (9) - but not in (10) - of the two words the co-existence of which occasioned Joxes's uncertainty ${ }^{4}$.

Irom this the conclusion is not, as suggested by Linsly, that Mates's criterion is too rigorous, but rather that it is based on a requirement which is simply irrelevant.

4.25 The most obvious constraint upon setting up $\underline{\sigma}$-characterizations and $f$-characterizations is that we need to be able to account plausibly for differences in the truth conditions of declarative sentences. The fact that there are comunication situations in which

(11) Jones is eating an apple

can be used to make a true statement, whereas

4. More exactly, there occurs in such cases surface structure syncretism involving underlying lexical items and their corresponding citation forms: ef. Harris 1970. 


\section{(12) Jones is eating a pear}

eannot so be used, needs to be accounted for in terms of a semantic difference between (11) and (12). Here a difference in truth conditions answers to a difference in meaning, most plausibly located in the nonequiform expressions apple and pear.

The crucial question, however, in the present context is the question when it is correct to account for the identity of truth conditions of declarative sentences by postulating their syponymity.

This is a question more often dodged than auswered, as e.g. by Katz \& Martin in their rejection of Ziff's scepticism about the synoxymity of

The tiger ate the man

and The man was eaten by the tiger ${ }^{1}$.

Ziff argued that since 'to eat a man is hardly the same as to be eaten by a tigex' and since what is said of the tiger in one sentence is accordingly not what is said of the man in the other sentence, then the claim that the two sentences are synonymous comes down to nothing more than that 'one is true if and only if the other is true'. Katz \& Martin concede the interdependence of truth conditions of the two sentences, but claim that this fact alone would not prompt us to say that the two are synonymous ${ }^{2}$.

1. Ziff 1966 p.231.

2. Katz \& Martin 1967 p. 488. 
Instead, however, of saying what would prompt them to call the two synonymous, they advance the somewhat bizarre (in the context) contention: 'Surely, the situation is the other way around: their synonymy prompts us to say that one is true if and only if the other is.' This riposte is simply irrelevant, in the first place because Ziff had not challenged the correctness of the thesis that truth values are preserved under substitution of synonymous expressions, and in the second place because that thesis cannot be advanced to settle the question whether or not two particular sentences in a particular natural language are synonymous. If The tiger ate the man and The man was eaten by the tiger are granted to be synonymous, then it follows that one is true if and only if the other is true. But what Ziff questioned was the grounds on which their synonymity should be 'granted'.

Katz \& Martin are no more convincing in their attempts to deal with Ziff's sentences Someone is a wife and Someone is a husband, and Someone ras a child and Someone was a parent. They assert (involking the authority of Chomsly and Abelard) that these pairs are synomymous and argue that, considered from the point of view of logical form, the difference between the first pair 'is only one of the order of existential quantifiers. For example, using $\underline{M}$ for "is a male", $\underline{\text { for }}$ fis a female", and IV for "is married to",' then Someone is a wife cowes out as 
$"(\exists \underline{x})(\mathrm{y})(\mathrm{Mx} \cdot \mathrm{My} \cdot \mathrm{Wxy}) "$, whereas Someone is a husband comes out as $(\exists y)(\exists \underline{x})(\underline{M x} \cdot \underline{M} \cdot \underline{W y}) "$. But this is to beg the question against Ziff by assuming that Someone is a rife means the same as Someone is a wire and someone (else) is her husband, while Someone is a husband means the same as Someone is a husband and someope (else) is his wife. (And why stop there? If all entailments count as part of the meaning of a declarative sentence, Someone is a wife ought also to be synonymous with Two people got married and someone was the vicar or priest or registrar or ship's captain....)

Since transformational semantics lacks the distinction between synthetic and analytic symonymity of sentences, it is not surprising that its apologists are forced to deny the relevance of differences of analytic meaning (which are the differences Zif ${ }^{\prime}$ 's examples raise). But that does not excuse the refusal to explain how symonymity oi sentences is established. For even if we were to dismiss differences of analytic meaning as irrelevant, that question still remains. It not only remains, but becomes pressing in viev of such concomitant assextions by Katz \& Maxtin as that Some triangle is equiangular and Some triangle is equilateral are 'true or false together', but that this is explained by 'geometrical theory', whereas the equivalence of Ziff's sentences is to be explained by 'semantic theory'. For just what no serious theory of linguistic 
knowledge can take for granted is a distinction between 'truths of geometry' and 'truths of English'. One might just as well subsume the fact that wives have husbands under the heading 'truths of matrimony'.

4.251 Cases usually cited as throwing doubt on the contention that differences of meaning are invariably associated with differences in truth conditions are certain types of conjunctive device and sentential adverb. Strawson observes that 'even sentences to which the notion of truth-conditions does seem appropriate may contain expressions which certainly make a difference to their conventional meaning, but not the sort of diffexence that can be explained in terms of their truth-conditions. Compare the sentence 'Tortunately, Socrates is dead' with the sentence 'Unfortunately, Socrates is dead'. Compare a sentence of the form ' $p$ and $g$ ' with the corresponding sentence of the form 'p but $\underline{g}$ '. It is cleax that the meanings of the members of each pair of sentences differ; it is far from clear that their truthconditions diffex' 1 .

A further class of cases is involsed by Lalsoff, who explicitly rejects what he describes as the usual assumption 'that two

1, Strawson 1970 p.11. 
utterances are synonynous if and only if they are identical in truth value'. Instead, he proposes that 'one must consider those sentences synonynous that could be used with the same truth value, IN IDIMPICAL INVIRONMHNIS' 2 . If we find that they cannot both be used in the same environment' we must suppose that they differ in meaning. The evidence adduced in support of this position comes mainly from the use of indefinite quantifiers in English. For example,

I wonder if Bill would lend me some money

and I wooder if Bill would lend me any money are said by Lalroif to be 'traditionally......considered synonymous' but in fact not to be so, since the rirst but not the second can be Pollowed by the explanatory sentence Then I can buy that new Beatle record, while the second but not the first can be followed by the explanatory sentence I already owe him a thousand dollars. According to Lalroff 'to exchange the two explanatory sentences in these cases would create two very odd utterances. Thus I conclude that in sentences of this type, at least, there is some difference in meaning between those containing some and those containing any.' It is clear from this example that what Lakoff means by the condition of occurrence 'in identical environments' is that in any environment in which the occurrence of a is not 'odd', substitution

2. Lalsofi 1969 p.610, n.2. 
of $\underline{b}$ for a must be possible without ensuing 'oddity'. By

'oddity' he means that 'in certain situations, confusion and misunderstanding will result'. Pairs of sentences not amenable to judgments of truth-or-falsity, e.g. interrogatives, Lakoff apparently considers to have the same truth value (i.e. presumably, none), and in such cases, therefore, occurrence in identical environments becomes the essential criterion. (Curiously, Lakoff counts conditionals as 'unfalsifiable'; but this need not concern us here.)

It way first of all be observed that the formulation of Lakoff's criterion does not quite correspond to his intentions as witnessed by the examples he gives. To insist on interchangeability in identical environments is in fact too strong a condition for Lakrof's purposes, since this will exclude otherwise 'synonywous' pairs which happen to differ in the ordering of surface elements. B.g. Zifít's

The tiger ate the man

and The man was eaten by the tiger

cannot both take, without ensuing oddity, the addition of the co-ordinate clause and promptly fell asleep. But it seems doubtful whether Lakoff intends to treat such pairs as nonsynonymous for that reason.

If we follow the spirit rather than the letter of Lakoff's criterion, it nonetheless supports some of Ziff's cases; e.g. 
it seems distinctly odd to follow the fixst, but not the second, of the pair

Someone is a wife

and Someone i.s a husband

with the co-ordinate clause and therelore someone must be a wife. On the other hand, application of the criterion does not seem to suppoxt Zifi's general scepticism about the synouymy of actives and passives. It is difficult to thinls of a context ix which e.g. John lrissed Mary will be odd, but not Mary was lrissed by John. However, not all of Lalroff's ixterpretations are eatirely convincing, because he has apparently overlooked a furthex some/any contrast, which involves the use of some fox contextually defined

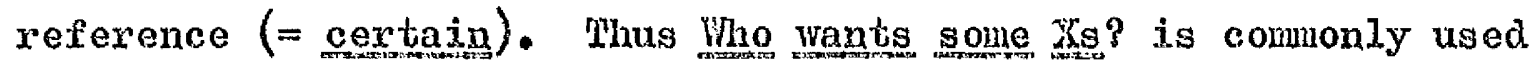
when the offex is quite specific (= I have some Xg: who vants them?) and contrasts with the speculative Iho wants any $\mathrm{Xg}_{\mathrm{g}} 3 \quad(=\mathrm{I}$ haven't any Xs: but I'll see if there are any available). Similarly, in If you eat some spinach, I'll give you ben dollars some may be taken as indicating that it is not just eating any old spinach that will earn ten dollars, but eating some particular spinach the spealrex has in mind. Lalroff, however, treats gome here as indicating the expectation that the person addressed wants ten dollax's' and implying that the speaker is offexing a reward, as opposed to

If you eat any spinach, 'll give you ten dollars where the only possible interpretation is that, for sone reason, 
the person addressed does not want to receive ten dollars, and that this sentence is a threat'. This seems dubious. A more natural interpretation of the latter sentence would be that the speaker offers ten dollars irrespective of what spinach is eaten.

The point makes some difference to Lakoff's thesis about synonyuy. For if we talse account of this particular some / any distinction, then it is doubtiful whether Lalroff has in fact produced any new examples of nonsynomymous declarative sentences having the same truth conditions. F.g. it may well be true that I wonder whether Bill will lend me some money (because the sum $I$ have in aind is considexable) but not true that I wonder whether he will lend my any money (because I know he never refuses a moderate request.) And so on.

4.3 We have thus far considered only incidentally the extent to which synonyny critexia must talse into account variations of linguistic and situational context. If it is conceded that expressions may have different meanings in different contexts, the possibility is open that two expressions may have the same meaning in one context but not in another, and hence the necessity arises for distinguishing between 'context-free synonymy' and 'context-bound synonymy'. (By 'coutext-fxee symonymy' is to be understood here synonymy independent of context, i.e. synonyny 
in all contexts.) A semantic description of $\mathrm{I}$ may be envisaged as accomodating such a distinction by adding supplementany qualifications to xelational characterizations. Thus, for exauple, a suitably qualified characterization of type $p^{1}$ might take the form:

$$
(\mathrm{a}=\underline{\mathrm{b}}) \mathrm{C}
$$

where ' $C$ ' denotes the context or set of contexts for which the semantic equivalence between a and $\underset{m}{\mathrm{~b}}$ holds.

4.31 The type of problem which arises in delimiting contextbound synonymities may be shown by means of an example. Suppose a semantic description of taglish distinguishes as least two meanings for the noun paper, one of which it shaxes with newspaper and the othex with essay ${ }^{1}$. Such a description encounters the problem of specifying the values of $\mathrm{Cl}$ and $\mathrm{C} 2$ for the following relational characterizations:

1. It is not essential to the example that these expressions should in fact have in Jnglish the meanings here attributed to them. 


$\begin{array}{ll} & (\text { papex }=\text { mewspapex }) \quad \mathrm{Cl} \\ \text { and } \quad & (\text { papex }=\text { essay) } \mathrm{c} 2 .\end{array}$

Any solution to this problem must presumably take into account at least the following three considexations.

(1) On some occasions when a sentence containing the word paper is used, the sentence is interpreted (and is intended to be interpreted) as would be a sentence identical but for the substitution of newspapex for paper; but $i t$ is so interpreted (and intended to be so interpreted) in vixtue of lnowledge about a particular communication situation. An example of this case would be the sentence The costs of publishing the papex are very high uttered by the editor of the local weekly at a Rotary club lunch.

(2) On some occasions when a sentence containing the woxd papex is used, it is doubtful whether it is to be interpreted as under the substitution newspapex for paper, or as under the substitution essay for paper, unless further information is obtainable about the relevant situation. An example of this case would be the sentence Professor Jones is reading his paper uttered in circunstances where the professor might conceivably be engaged in eithex of two activities, namely (i) addressing a learned society, or (ii) catching up on the day's news. 
(3) On some occasions when a semence containing the word paper is used, no such doubt arises because the rest of the sentence makes the appropriate interpretation cleax. Tramples of such cases would be uttexance of the sentences The papex ceased publication and the paper was on the mating habits of the giraffe.

Various answers may be envisaged to the question as to how a semantic description should deal. with facts such as these. It will be assumed, for purposes of the example, that the linguist is able to verify empirically under what circunstances doubt axises about the intexpretation of words in particulax communication situations, and this information may thus be looked upon as constituting part of the data available for the construction of a semantic description of $\mathrm{L}_{\text {. }}$ The question to be considered, therefore, is the status of this evidence as warranting ox not warranting the incorporation of possible features in the description.

One possibility is that facts of the kind described in (1) should be accounted for in the description by an appropriate specification of $\mathrm{Cl}$, e.g. to the effect that papex and newspaper axe symonymous in sentences uttexed by journalists. This course would be objected to by theorists who, lilse Katz \& Fodox ${ }^{2}$,

2. Thatz \& Todox 1963. 
claim that a semantic description of natural language cannot be expected to account systematically for the ways in which variation of situątion, or 'socio-physical setting' affects the interpretation of sentences. On the othex hand, it would - at least in principle - be supported by theorists who, like Lyons, claim that an identification of the relevant situational context is an essential part of the establishment of instances: of synonymity.

The difference between (1) and (2) seems to be that in the former case but not the latter the appropriate interpretation of paper is determined by features of the communication situation lnown to participants in that situation. The difference between (1) and (2) on the one hand and (3) on the other seems to be that in (3) alone the appropriate interpretation of paper is determined by the linguistic context independently of situational features of the communication situation. Here, i.e. in (3), there would presumably be agreement between followers of llatz \& Fodor and followers of Lyons that a semantic description should account for the appropriate interpretation of paper by an indication of its contextual symonymity with newspaper and with essay in the appropriate cases. However, the argwuents which might be adduced for and against these solutions require closer examination. 
4.32 According to Lyons, 'once we accept that synonymy must be bound to context, it ceases to be a theoretical problem at a11' 1 , and in Structural Semantics a procedure is proposed for establishing instances of synonymity on the basis of interchangeability in a particular linguistic context (defined by a set of sentences of the language) and a particular situational context ox range of contexts ( $e . g$. 'malsing a purchase in a shop'). The linguist is envisaged as examining empixically how the speakex's comitment vaxies under modification of sentences in certain ways, white holding the situation-type constant. This procedure is held to determine a relationship which Lyons calls 'praguatic implication', and knowledge of synonymy is explicated as knowledge by the spealser of certain 'pragmatic implicational' connexions between the utterance of certain sentences in certain types of situation.

For the dercription of natural languages accessible to experimental investigation by the linguist, Lyons would progose to establish an instamce of synonymity as follows:

1. Iyous $1963 \$_{4.46}$. It is not quite clear how this statement is to be taken, i.e. what theoretical problems we are invited to see as being solved by accepting that synonymity must be bound to context. 
'Let us take two utterances which differ poxmally in one respect only and let that difference consist in the occurrence of a form a in the one utterance at the point at which an item $\underline{b}$ occurs in the other: We have a wide range of cigars and We have a wide selection of cigars. These two utterances, we will suppose, have been heard in a tobacconist's shop, and we are interested in seeing whether the forms range and selection are synonywous here. (our reason for thinking that they might have the same meaning could be based on a 'hunch' or even on the statement to that effect by the native lnglish spealrer.) The test whereby we establish that two complete utterances of this kind are synonymous is, in principle, behavioural and empixical.

'The first problem is to delimit the context of situation. There is no need to exaggerate the theoretical difficulties of this... Let us here assume that the linguist has provisionally identified as the same situational context (itself 'culture-bound') the events and activities which constitute making a purchase in a shop. Whe linguist can satigry himself that the utterances We have a wide range of cigar's and We have a wide selection of cigars occur in this context, if he is thorough in his methods, by going around and exasperating several: tobac- 
conists with his 'informant-wechnique'. He can then try the effect of substituting different items in place of cigars. Assume that he has collected a number of utterances of the form We have a wide (raage/selection) of ( $)$; that is, a number of utterances in which range and selection are interchangeable, and therefore have meaning..., The question now is to decide whether the forms range and gelection have the same meaning in these utterances. This does not consist simply in asking the informant; for this would be merely to invite him to invent some difference - say, for example, that the use of gelection implies that he has chosen his stock with care. But the problems connected with testing the informant's response to the substitution of the two items in the frame, though they exist, are problems of a practical nature, as are the problems of devising tests for identifying vaxiants in phonology. The aim is to inveigle the informant, without prejudice to the issue, into accepting or refusing to accept utterance a as a 'rep-

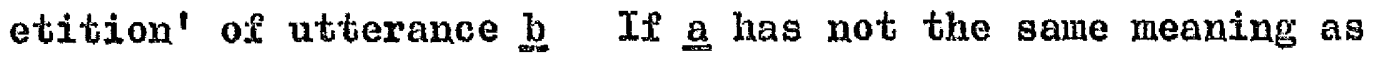
b it will eithex not imply something which b iuplies or imply something which b soes not imply. And it should not be beyond the practical ingenuity of the linguist to discover this. ${ }^{2}$ 
What wight well be questioned in conmexion with Lyons's method, howevex, is whether it is not beyond the parctical ingenuity of the linguigt to determine systewatically all the types of situation in which a contextm-bound synonymity holds. 3 If a serious attemgt were made to delimit all the situations in which paper and newspapex receive identical interpretations, it would doubtiless soon become apparent that a very complex weighting of the impoxtance of different factors in the situation operates. It is highly unlikely, for example, that a critexion as simple as 'uttexance by journalists' will. turn out to be correct. (If it were, then the semantic description of Inglish would lead us to expect that an appropriate response to the eminent joumalist who, about to address a learned society, announces 'I have mislaid my papex' might be 'Don't worry: there's time to go out and buy another. 1) Nonetheless - the theorist of contextmbound synonymy might axgue -. the pxactical dificulty of the extexprise of discovering in exactily what situations two words axe synonymous does not impugn the validity of the concept.

3. Katz \& Fodor adduce the fact brat it is practically impossible to produce a systematic analys is of all conceivable ways in which variation of 'socio-physical setting' may affect the intexpretation of sentiences as a reason for rejecting the view that a semantic theory should take situational context into account (Katz \& Fodor 1963 p.489). 
4.321 We may pursue the matter further by considering the following proposal :

(K) ' $\underline{a}$ and $\underline{b}$ are context-bound synonyms in contexts of type $C$ if and only if in contexts of type $C$ the substitution of one for the other does not affect the conmitment of the spealser'.

It is of interest to examine what the consequences of a strict application of such a criterion vould be in the construction of our semantic description of $\mathrm{L}$.

The first consequence we may note is that equivalence in the force of speech acts ${ }^{1}$ becomes a suficient condition of synonynity, since the criterion proposed leaves the linguist free to set up as context-bound synonyms any two expressions for which a context-type $c$ can be established such as to perwit the substitution of expressions in certain sentences without affecting the comnitment of the speaker in that context. Thus considex the case of Picasso, who, called upon to identify a forgery, has the choice between uttering the sentences this is not a Picasso and This is not a work of mine. In this context the two sentences, and the two constituents a Picasso and a work of mine, way be regarded as context-bound synonymous expressions. It rould be unavailing to object to

1. On the force of speech acts, see below $\mathrm{Ch} .5$. 
this proposal that the commitment is different in the two cases, since in saying the forwex Picasso does not commit himself explicitly to the proposition that the work is his, while in saying the latter he does not commit himself explicitly to the proposition that it is by Picasso. This difference would indeed be relevant if we were being asked to consider the sentences in abstraction from the situational context; but under $\mathrm{K}$ what we axe asked to attend to is, quite specifically, what Picasso cowmits hinself to in that situation. In short, if pairs of locutionary acts are equivalent in foxce in a certain type of context, we may count the expressions which contrast as contextbound synonyms. (If we were pedantical ly inclined, we could of course require Picasso to identify the forgery by uttering some such formula as I, Picasso, certify that this is not a work of mine. But this alters nothing, since the following formula will do just as well: I, Picasso, certify that this is not a Picasso.)

But this has the following consequence. If in defining a situational context or range of situational contexts it is pexmissible to include a necessaxy condition $Q$ such that $Q$ specifies the relation of the speech acts in question to particular language-users or particular times and places, then there is no guarantee that a pair of context-bound synonyws will not include a deictic and a non-deictic member. Now any 
theory of synonymy which allows this may require us to concede e.g. that 'my-Fnglish' is semantically different from 'yourEnglish', that the Dnglish spoken on the first of January is not the same language as the Inglish spolsen on the second of January, etc. The proposed criterion, in other words, permits the distinction within a 'language' of indefinitely many situationally-bound 'sublanguages', each with its own semantic rules and synonyms.

There is, it might perhaps be urged, nothing methodologically vicious about that, provided we are prepared to foxggo generalization 2. But unfortunately there is a further consequence, namely the failure to distinguish semantic from extralinguistic lnowledge. This vitiates the method even in the investigation of the most strictly delimited sublanguage. The argument in support of this criticism is simply that if the intexpretation of an expression depends on certain features of the communication situation, as distinct from the expression used, then to that extent the interpretation is not a matter of semantic knowledge. Whus, to talre Lyons's example, suppossing the linguist's investigation sis confined to the semantics of 'shopping Inglish', or, moxe strictly still, to the semantics

2. Nonetheless, quot howines tot linguae seems a curious theoretical basis for linguistics. 
of 'tobacconist's Bnglish', even then the mothod proposed offers no sound basis for reaching the conclusion that e.g. range and selection are (or are not) synonymous. For it incorporates no way of digtinguishing between the respects in which the speakex's commitment is based on lnowledge of certain facts about the situation, and the respects in which the speakex's conwitment is based on lonowledge of the meanings of the words used. To lnow that $X$ is a tobacconist, ox a journalist, or a bank manager, or to know that a conversation is taking place in a tobaccouist's shop, or a ners-o paper office, or a bank, is to know - so the axgument would run - something about the world in which one lives, not something about the language one speaks. If the purpose of a semantic description is to account for communication by postulating semantic knowledge shaxed by language-users, it requires a method of investigation which distinguishes evidence of semantic lenowledge from evidence of extralinguistic knowledge.

4.322 The objection that criterion IS involves a failure to distinguish questions about the force of speech acts from questions about the meanings of expressions may also be pressed on the basis of examples like the following. 
Consider the :sentences:

(1) Yes

(2) I was in Bognox Regis on the lst of January 1967

(3) I would not be telling the truth if I denied it

The speech act of confirming my presence in Bognor Regis on the lst of January 1967 when asked Were you in Bognor Regis on the lst of January 1967? can be realized by my uttering (1), (2) or (3). But to say that this constitutes evidence of the context-bound synonymity of (1), (2) and (3) would be quite plainly to confuse two different senses of 'meanj.ng', i.e. confusing what the words 'mean' with what someone's uttering them 'means'. The special context does not confer synonywity upon (1), (2) and (3), any more than other contexts render them nonsynonymous. What the context does is afford we the choice of meaning the same thing by uttering (1), (2) or (3).

4.323 The further problem of determing what a speake $\mathbf{r}$ is 'committed to' by uttering a sentence in a given context may be illustrated by an example given by Bennett:

(8a) At this moment he's on a train going to London

(8b) At this moment he's in a train going to London. Setting aside, for purposes of the example, the sowewhat extraordinary possibility of interpreting (8a) as meaning that he is on the roof of a train going to London, we may ask: are $\pi$ 
on and in here context-bound synonyms ? But,first of all, are (8a) and (8b) symonymous in the context ? Bennett is inm clined to regard this as a case of 'coextensiveness' rather than synonymity: fox, he axgues, these semtences 'would noxmally describe the same situation, but it would be countexintuitive to postulate some higher-level linguistic unit that can be realized alternately as on and in, since on-ness and in-ness are essentially different notions, $i . e \cdot$ on ${ }^{\underline{s}-t}$ and in $\underline{s}-\underline{t}$ diffex in cognitive meaning. What we have, rather, is a case of coextensiveness. There are certain situations in reality that can be perceived by a spealser of Bngligh as involving either on-ness ox in-ness. One such situation is the boarding of a train, which can be pexceived as getting onto it or getting into it. Thus whether one uses on or in in (8), the situation described is the same.' 1

Setting aside the stratificationalist trappings, what Bennett's answer comes down to is that $(8 a)$ and $(8 b)$ difier in meaning, but in such a way that the diffexence is always $i x-$ relevant in the context. Since stratificational theory leads him to hold that 'synonymy depends on the realizational phenomenon lnown as 'diversification' 12 , he is inclined to reject the synonymity of (8a) and (8b) because he cannot inagine a plausible

1. Bennett 1968 p. 164 .

2. Benuett 1968 p. 159. 
unit which could here undergo 'diversification'. This may be good stratificational theorizing, but it is not convincing linguigtic analysis. For tio approach the problem in this way is to assume the availability of (nonlinguistic) criteria for deciding questions of comceptual classification. The nature of these criteria is allowed to remain obscure, e.g. we are not told why 'on-ness' and 'in-ness' are 'essentially different notions' 3 . But worse still, the argument propounded simply assumes what needed to be demonstrated, namely that to be on a train and to be in a train here correspond to a 'genuine' conceptual difference. Precisely what is at issue is whether English recongnizes a difference, not whether in the abstract a difference might conceivably be recognized.

On the other hayd, the application of criterion II yields no happier solution, since it is not clear what evidence we would look for to decide whether a speaker takes on different 'commitments' in the context of describing X's whereabouts by uttering (8a) and (8b). And if this is not clear, it is because the notion of 'commitment' itself is not clear.

3. This is a puzzling assertion. It is not as if it would be out of the question to imagine a language which simply did not malre the English distinction between on and in, but had only a single locational preposition translating both. 
433. More remains to be said about the validity of setting up context-bound synouyms as a way of accounting for instances where a choice between interpretations is resolved by the linguistic context, independently of situational features. Reference has already been wade to the rejection by Katz \& Todor of the thesis that a semantic description should take situational variation into account. Having pointed out that the possible ways in which situational factors may affect the interpretation of sentences are virtually limitless (hence not amenable to systematization), they conclude that a semantic description is adequate if it accounts only for interpretational vaxiations dependent on the linguistic context.

The basis of this view is that 'except for a few types of cases, discourse can be treated as a single sentence in isolation by regarding sentence boundaries as sentential conpectives.' 1 Katz \& Fodor argue:

'As a matter of fact this is the natural treatment. In the great majority of cases the senteace break in discourse is simply and-conjunction. (In others, it is but, for, ox, and so on.) Hence, for every discourse, there is a single sentence which consists of the sequence of m-sentences that comprises the discourse connected by the appropriate sentential connectives and which exhibits the same semantic

1. Katz \& Fodor 1963 p. 490. 
relations exhibited in the discourse. But since the single sentence is, ex hypothesi, described by a theory of semantic intexpretation, in every case in which a discourse can be treated as a single sentence, a theory of semantic interpretation is as descriptively poweriful as a theory of setting selection. 12

In support of this view, Katz of Fodor adduce the following considexations.

'To illustrate this, let us consider the two-sentence discourse: "I. shot the man with a gun," "If the man had had a gun too, he would have shot we fixst." The first sentence of this discourse is ambiguous in isolation, but not in this setting. But the problem of explaining this disambiguation is the same as the problen of explaining why the single sentence "I shot the man with a gux, but if the man had had a gun too, he would have shot me first," does not have an ambiguous first clause. Likewise, consider the discourse, "I heard the noise," "The noise was completely inaudible," and its single sentence equivalent, "I heard the noise, and the noise was completely inaudible." In showing why the single sentence is anomalous, a theory of semantic interpretation exhibits precisely those semantic

2, IKatz \& Todor 1963 pp. 490-491. 
relations in which the anomaly of the discourse resides. This technique of replacing discourses or stretches of discourses with single compound sentences, by using sentential connectives in place of sentence boundaries, cleaxly has a very extensive application in reducing problems of setting selection to problems of semantic intexpretation o: sentences in isolation. Thus, given a theory of semantic interpretation, it is unclear how wuch is left for a theory of setting selection to explain. 3

Katz \& Fodor concede that not all discourse can be converted straightiforwardly into single sentences, but do not see this as a serious objection.

'For example, the digcouxse "How axe you feeling tomay?" "I an fine, thanks" does not convert to "*Fow are you feeling to-day and I an fine, thanls" because the compound sentence is ungramutical. But the fact that sentences of different types cannot be run together in the obvious way may not pose a serious problem because it is not at all clear that less obvious convexsious will not lead to a satisfactory treatnent of such cases within a theory of semantic interpretation. For example, we way convert the discourse jugt cited into the single sentence, "X asked,

3. Katz \& Todor 1963 p. 491 n. 12. 
' 'How are you feeling today?' and Y replied, 'I am fine, thanks.'" If such conversions can be carried out generally, then any problem about disambiguation, detection of anomaly, and so on that can be raised and/or solved in a theory of setting selection can be raised and/or solved by reference to an analogon in the theory of semantic interpretation. But even if such conversions cannot be carried out generally, the most interesting and general cases will still be within the range of a theory of senantic interpretation. 14

Thus in a semantic investigation of the kind envisaged by Katz \& Fodor setting up context-bound synouyms would be one possible way of accounting for the fact that two sentences which do not receive the same semantic interpretation in isolation do so when forming part of certain longer discourses. For example,

(1) Sraith read his paper

and (2) Swith read his newspaper will be, we way assume given different interpretations in isolation by a semantic description of English, since they have different truth conditions. But considered as first sentences in the two-sentence discourse formed by the addition of, say,

(3) He looked ifirst of al1 at the stop-press on the back page

4. Katz \& Fodor 1963 p. 491 n.12. 
they receive, let us assume identical interpretettions. If Katro \& Fodor's proposals are accepted, these two-sentence discourses may be treated as the compounds

(4) Smith read his paper, and looked first of all at the stop-press on the back page

and (5) Smith read his newspaper, and looked fixst of all at the stop-press on the back page. Accordingly, one possible way of accounting for the interpretation of (4) and (5) would be postulation of the synonywity of paper and gewspapex is certain contexts, the contexts in question being defined so as to include (4) and (5).

4.331 Setting up context-bound synonymities is also a possible way - although not necessarily a theoretically desirable way - of dealing with certain other types of case. For example, the sentences

(6) John grows plowers

and (7) John grows roses

will, we may assume, be represented by a semantic description of Inglish as having different meanings in isolation. Consider, however, the two-sentence discourses comprising (6) followed by (8) and (7) followed by (8): 
(8) Roses are the only flowers John grows.

These two-sentence discourses can be treated as the compounds

(9) John grows flowers, and roses are the only flowers John groirs

and (10) John grows roses, and roses are the only flowers John grows.

It is relevant to note at this point that if we follow Lyons then, given appropriate informant response, both (4) and (5) and also (9) and (10) can be established as containing context-bound synonyms. The expressions paper and nerrspaper will be context-bound synonyms in the frame 'Smith read his , and looked first of all at the stop-press on the back page'; while flower and rose will be context-bound synonyms in the frame 'John grows s, and roses are the only flowers John grows' 1 .

1. It suffices under Lyons's proposals to establish that (9) 'pragmatically implies' and is implied by (10). Pragmatic implication and pragmatic equivalence are defined in terws of 'assertion' and 'denial'. E.g. in the case of antonyms such as single and married, 'the denial of either one is implicitly equivalent to (implies and is implied by) the assertion of the other. (More precisely, the denial of a sentence containing either one, $\mathrm{S}_{1}$, is equivalent to the assertion of a sentence, $\mathrm{S}_{1}$, containing the other.)' The linguist establishes this by investigating responses of informants. He will find, for instance, that the informant, having asserted a sentence of the form $X$ is single will reject a sentence $\mathrm{K}$ is married - perhaps adding indignantly I said once that he Wrasn't, or something of the sort.' (Lyons $1963 \mathrm{pp} .88-39$ ) In the present example, the informant, having asserted either of the pair (9) or (10), is committed under pain of inconsistency to the assertion of the other. 
Now a semantic description of the type envisaged by Katz \& Fodox wight deal with (9) and (10) in one of three possible ways. The first possibility is that (9) and (10) are represented as semantically different (i.e. as having one or more readings not shared). The second possibility is that (9) and (10) are represented as semantically equivalent (i.e. as sharing all. readings) in virtue of the fact that llower and rose, al though not in jisolation symopymous, are in certain contexts (of which (9) and (10) are examples) assigned the same reading. The third possibility is that (9) and (10) are represented as semantically equivalent in virtue of an operation of the relevant projection rules, whereby the difference in meaning between flower and rose is not allowed to contribute a difference in meaning to the eventual readings for the sentences (9) and (10).

An examination of these three possible solutions shows that whether to set up flover and rose as context-bound synonyms must depend on the answer to the prior question whether (9) and (1.0) are synthetically synonymous. If they are not, it follows that the description should not be allowed to represent $210 w e r$ and rose as synonymous in this context. But no way of axriving at the crucial decision is proposed by Katz \& Fodox, since they do not offer external criteria for synomymity. Iyous's proposals, on the other hand, offer a solution, but manifestly the wrong one, since that would not distinguish the case of (9) and (10) from the case of (4) and (5). 
4.332 It should be made clear at this point that the rejection of a notion of 'context-bound synonymy' which treats the two cases alike is not based on a covert argument frow 'intuition'. It might pexhaps be urged that it would be counterintuitive to propose a solution which treated rose and plower as synonyms in (9) and (10), but intuitively satisfactory to treat paper and newspaper as synonymous in (4) and (5). But we do not need to rely on intuitive satisfaction here, for there is a reason for distinguishing the two cases whicir does not appeal to intuitions at all. The paix (9) and (10), unlike the pair (4) and (5), falls under a general rule for Inglish whereby the truth of the conjunction of a sentence 'only xs are P'and a sentence

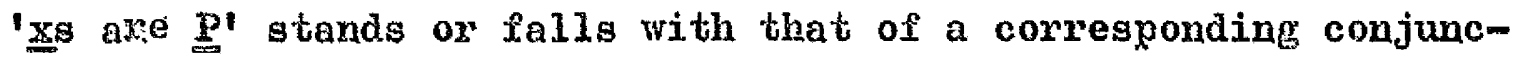
tion of sentences 'ys are $\mathrm{P}^{\prime}$ and 'only $\mathrm{xs}$ are $\mathrm{P}^{\prime}$, provided $\mathrm{xs}$ are a proper subset of $\mathrm{ys}{ }^{1}$ In such cases, the postulation of a contest-bound synonymity is otiose, i.e. it does not contribute to an explanation of the facts to suppose that a particular relation (context-bound synonymity) holds between two expressions, when the facts are already explained by a general rule which does not require that supposition. No such consideration applies to (4) and (5), where the postulation of a context-bound synonymity provides an explanation which cannot be derived from a corresponding general rule.

1. Here $\underset{\text { s }}{ }$ are roses, $\mathrm{Ys}$ are flowers and $\mathrm{P}$ 'grow by John'. 
4.333 An example where criterion $\mathrm{K}(4.321)$ leads to a postulation of symonywity which conflicts with a better linguistic explanation of the case would be:

(11) No-one was in the room with John

(12) No-one was in the room except John.

Here criterion $K$ gives with and except as context-bound synonysas, a solution which is obviously wrong in view of such evidence as e.g. that we can have beside (12), without changing the meaning, No-one except John was in the roow, whereas No-one with John was in the room is not equivalent to (11).

4.334 Further, consider the sentences

(13) Filary climbed Everest

and (14) Milary climbed the highest mountain in the world. If we assume that a semantic description of Higlish will exhibit these sentences as having different meanings in isolation, the question arises how to represent the interpretation of the two-sentence discourses comprising (13) preceded by (15), and (14) preceded by (15) :

(15) Bverest is the highest mountain in the world. These two-sentence discourses can be treated in the fauiliar way as the compounds:

(16) Bverest is the highest mountain in the world and Hilary climbed Trerest. 
and (17) isverest is the highest mountain in the world and Iilaxy climbed the highest mountain in the world.

Now if a speaker is identically conmitted by an assertion formulated either in the way represented in (16) or in the way represented in (17), then it follows by criterion $\mathrm{K}$ that Everest in its second instantiation in (16) and the highest mountain in the world in its second instantiation in (17) can be treated as context-bound synonyms. This seens to be the case, since it would be inconsistent for a speaker to affirm (16) but depy (17), or vice versa.

However, it is an adequate justification of the spealser's 'commitment' in this case that Isverest and the highest mountain in the rorld be granted to refer here to the same unique entity. In other words, the required condition is merely that there is just one $x$ such that $(i) \underline{x}$ is called 'Dverest', and $(i i) \underline{x}$ is the highest mountain in the world. Thus the 'context-bound synonymity' here assures us only of identity of reference, which is inadequate. Any theory of synonyny which allows this must be rejected, since it fajls to distinguish between what is referred to by the use of an expression in a certain context or contexts, and what the expression means. 
4.4 Context-bound synonyny of the kind where the context in question is situational is a notion which seems unlikely to be helpful when it comes to explaining how a speaker's knowledge of synonymy enables hin to detect the analyticity of certain sentences, for the obvious reason that the feature of analytic sentences is that their truth value is independent of oontingent matters of fact, and therefore of the context of situation in which they are uttered. Here at least, it appears, the construction of a semantic description of Lisrequires is to be able to set up $p$ - characterizations without situational limitations.

4.41 It is not perhaps clear whether all natural languages afford the possibility of characterizing a statement as analytic ${ }^{1}$, but it is at least clear that many of them do. Such languages may be considered to be 'nonextensional' languages in the sense defined by quine ${ }^{2}$, i.e. as possessing devices equivalent to a modal adverb 'necessarily' which yields truth when and only when applied to an analytic statement.

1. It is, in any case, possible to imggine one into which one would have difficulty in translating English sentences characterizing a statement as analytic (Harris 1967).

2. quine $1961 \mathrm{pp}$. 29-30. 
In the sentences of such languages, interchange of expressions having identical extension does not guarantee preservation of the truth of a statement. Quine designates the stronger relation which must hold between expressions in order to preserve truth values under substitution in the sentences of nonextensional languages 'cognitive synonymy' 3. His argument is:

'Intexchangeability salva veritate is meaningless unless relativized to a language whose extent is specified in relevant respects. Suppose now we consider a language containing just the following materials. There is an indefinitely large stock of one-place predicates (for example, ' $\underline{5}$ ' where 'Px' means that $x$ is a man) and many-place predicates (for example, ' $\underline{G}$ ' where ' $G x y$ 'means that $\underline{x}$ loves $y$ ), mostly having to do with extralogical subject matter. The rest of the language is logical. The atomic sentences cousist each of a predicate followed by one or more variables ' $\mathrm{x}$ ', ' $y$ ', etc.; and the complex sentences are built up of the atomic ones by truth functions ('not',

3. The term 'cognitive' is here intended to rule out of consideration whether the expressions in question have the same 'associations', the same 'emotive value', the same 'poetic overtones' etc., and to indicate that one is concerned simply with the use of words in expressing statements to be judged true or false, and as conforming or not conforming to the patterns of valid inference. 
'and', 'or', etc.) and quantification. In effect such a language enjoys the benefits also of descriptions and indeed singular terms generałly, these being contextually definable in known ways. Bren abstract singular terms naning classes, classes of classes, etc., axe contextually definable in case the assumed stock of predicates includes the two-place predicate of class membership. Such a language can be adequate to classical mathematics and indeed to scientific discourse generally, except in so far as the latter involves debatable devices such as contraryto-fact conditionals or modal adverbs like 'necessarily'. Nor a language of this type is extensional, in this sense: any two predicates which agree extensionally (that is, are true of the same objects) are interchangeable salva veritate. 4

It follows, according to Quine, that unless there is an assurance that $\mathrm{y}_{\mathrm{i}}$ is nonextensional interchangeability galva veritate is too weals; for that bachelor and unmarxied man are interchangeable salva verttate in an extensional language guarantees no more than that All and oply bachelors axe unmarried men is txue. 'There is no assurance here that the extensional agxeement of 'bachelox'

4. Quine 1961 p. 30. 
and 'unmarried man' rests on meaning rather than merely on accidental matters of fact, as does the extensional agreement of 'creature with a heart' and 'creature with kidneys'. 5 However, 'if a lamguage contains an intensional adverb 'necessarily'...or other particles to the same effect, then intexchangeability salva veribate in such a language does afford a sufficient condition of cognitive synonyny'. 6

The argument can be adapted to fit various types of expressions, including proper names and sentences. Two declarative sentences $\underline{a}$ and $\underline{b}$ in a nonextensional language are cognitively synonymous if interchangeable salva veritate within biconditional formulations of the type 'Necessarily, a if and only if $\underline{b}^{\prime}$.

4.42 Since we canmot assume that nonextensionality is a linguistic universal, Quine's argument leaves us asking the question: how about extensional languages? For no reason has been offered for rejecting identity of extension of predicate expressions as a sufficient condition of L-synonymity where \lfloor is an extensional language. In order to show that this possibility too nust be rejected, it is relevant to divido Quine's class of extensional languages into two subclasses, which might be termed 'axtensional $1 '$ (or 'yl') and 'Hxtensional 2' (or'W2'),

5. Quine $1961 \mathrm{p} .31$.

6. Quine 1961 p.31. 
according to whether or not in any such language it is possible to formulate an analytic truth other than a logical truth. We should thus have a three-level hierarchy of languages. At the'lowest' level (52') are lamguages in which logical truths are the only nonsynthetic truths which can be formulated . At a 'higher' level ('El') are languages in which logical truths and other analytic truths can be formulated but camnot be characterized as analytic. At the 'highest' level ('nonextensional') are languages in which logical truths and other analytic truths can be both iormulated and also characterized as analytic (by means of such devices as the modal 'mecessarily').

If now, by ilat, we remove from the Dnglish language all devices equivalent to quine's modal 'necessarily', but make no other changes, we are left with a language which qualifies as extensional, $i . e$. is such that in no shape or form can ve translate into it a proposition to the effect that such-and-such is necessarily true. Let us call this language 'Bnglish-in'.

Let us next consider the question whether Laglish-W is an Tl language or an $\mathrm{T} 2$ language, and let us grant for the salke of the argument (a) that in the world of speakers of English-I no bachelors are married, nor are there any unmarried men who are not bachelors, and (b) that in the world of speakers of Baglish-i no creature with a heart is kidneyless, nor are there creatures with kidneys but without a heart. 
Now to say that inglish-D is an 12 language will be to maintain that the statements expressed by the Inglish-1 sentences

(1) All and only bachelors are unmarried men

and (2) All and only creatures with a heart are creatures with kidnevs

are either false or, if true, nonanalytic. But neither statemeat, ex hypothesi, is false: therefore both wust be held to be nouanalytic.

But if both (1) and (2) are true but nonanalytic, a semantic description of English-F must include the relational characterization

\section{(sp i) 'bachelox $\neq$ unmarried man'}

since if bachelor and unmarried man have the same meaning in Dnglish-15, (1) states a truth guaranteed by the meanings of the words, i.e. an analytic truth. Thus for an I2 language identity of extension of expressions will not be a sufficient condition of synonymity •

However, there seems to be no reason which precludes the supposition that the semantic description of English-i could include the following relational characterizations

$$
\begin{aligned}
& \text { (sf ii) 'bachelor }=\text { unmarried man' } \\
& \text { (Sp iii) 'Lheart }=\underline{\text { lkidney' }}
\end{aligned}
$$

(granted appropriate substantive characterizations to guarantee the internal consistency of the description). 


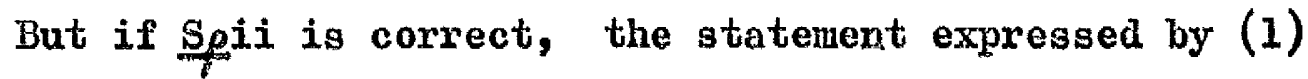
is analytic, since its truth is guaranteed by the meaning of (1), the negation of which is self-contradictory. It follows that Dnglish-iD is an 31 language, since it affords the possibility of foxmulating an analytic truth other than a logical truth. If Spiii is correct, then creature with a heart and ereature with kidneys are, if semantically endocentric, nonsynonymous. But in that case, identity of extension does not guaxantee symonymity of expressions in an El language, since the pairs bachelor and unmarried maan, and creature with a heart and creature with kidneys are each ex hypothesi co-extensional pairs in thgligh-li.

It follows that whether Buglish-i is an Dl language or an D2 language, in neither case is identity of extension a sufficient condition of synonymity of expressions. But since English-I must be one ox the other, identity of extension has been shown to be an inadequate criterion of synonymity for exteusional languages.

4.43 This still leaves unanswered Quine's question about the source of the assurance that the agreement between certain coextensional terms 'rests on meaning rather than merely on accidental matters of fact'. For the descriptive linguist con- 
cerned with the analysis of natural languages, the assurance must ultimately lie in empirical tests of some kind. The problem may therefore be put in the form: what lxind of test conld give this kind of assurance ?

4.431 Failure to deal with this question vitiates IKatz's claim ${ }^{1}$ to have solved precisely the problem of distinguishing analytic and syathetic truths zaised by W. V. Quine'. In Katz's analysis, the chaxacterization of a particular sextence as analytic or synthetic depends altimately on the semantic descriptions assigned to individual words in it. But since no account is given of the method for assigning semantic descriptions to individual words, the explanatory force of Khatz's 'solution' is nil ${ }^{2}$.

When, in a latex paper 3 , Kotz addresses himself specifically to the question of empixical tests fox detexinining the analytic sentences of a natural language, he offers the following proposal:

1. Katz 1964.

2. It needed at least to be shown that the evidence fox assigning semantic descxiptions to words was independent of the assessment of sentences containing them as 'synthetic' or 'analytic': othexwise the denonstration of the distinction must be circular.

3. Katz 1967b. 
We present spealsers with short ligts of sentences. list A containg only sentences that are clear cases of what we would regaxd as analytic. Lists $B_{8} \mathrm{C}, \mathrm{D}$, etc. contain cleax cases of sentences that axe not analytic, but, say, xespectively, synthetic, contradictory, anomelous, etc. Then, we give the spealrers a batch of semtences of all sorts and ask them to place these on the ligts to which they belong. Bach sextence is to be put on the list with whose members it is similar. If this experiment is conducted propexly and if the predictions that the semantic component of the gramax inakes watch the actuaj. sorting performed by the speaker's (cases that are put on list $A$ axe those and only those that axe predicted to be analytic, and so on), then we can claim that we have evidence, obtained in a quite unobjectionable fashion, in favor of the semantic component, as a result of its successful predictions about the data. However, the qualification that the expeximent be conducted propexly is extremely important. If the controls used in the expeximent en- 
sure that the members of the short listis $A, B, C$, etc. are sufficiently different from one another in the appropriate respects, then there will be no spurious common features that might lead spealers to classify sentences on the basis of irrelevant linguistic properties (e.ge in the case of list $A$, on the basis of some linguistic property other than analyticity). Positive results in this experiment can be interpreted to mean that the judgments of the speakers reflect a recognition of the analyticity of the sentences concerned. We can say, then, that our definition of analyticity, which enabled us to predict the outcome of the experiment, describes the concept of analyticity employed by the spealrers as their implicit critexion for identifying analytic sentences, i.e. for differentiating those of the test sentences that are similar to the members on list A from those that are not similar to them. We can say this on the grounds that assuming that this is their criterion provides us with the best explanation of the behavioral data obtained in the experinent.' 4

4. Katz 1967b pp.50-51. 
But this will not do either. Obviously, for any $\mathrm{x}$, if $\mathrm{E}$ is an example of $x$ we can construct informant tests designed to elicit further ingtances judged to be 'like 忽' A test based on this principle, howerex, falls short of what is required in at least three ways. First, as Quine in his bxief rejoinder to Katz points out different lists will presumably be required for English and for e.g. French, but 'no linguistically general method is offered for making such lists. 5 . Second, even fox one language the test is in principle inadequate unless there is a prior guarantec that list $A$ contains all the possible types of analytic sentence in the language, and only those. But where does this guaxantee come from ? For the problem under discussion is precisely how to draw up such a list. If only what Katz calls 'clear cases' are included in the list, the test is simply a test of lilseness to 'clear cases', not a tegt for delimiting the class of analytic sentences。 Thirdly, nothing assures us that the analytic

5. Quine 1967 p. 53. 
gintences of a natuxal language will have in coumon only theix analyticity; consequently, Katz's test is not powerful enough to reveal what the criterion of analyticity is, but only powerful enough to elicit from informantis a set of sentences judged to be 'like other (ex hypothesi analytic) sentences'.

Thus a 'likreness' test offers no answer relevant to the question of distinguishing analytic from symthetic; rather, it assumes that distinction already drawn. Indeed, unless it vere already dram, the claim that the semantic component is able to 'predict' analytic sentences would lack substance. But as long as the method of constructing senantic characterizationg for indjvidual words remains obscure, no explanatory adrance has been made. For it is there that a cleavage between sematic and extralinguigtic lnowledge must fixst be introduced if we are dealing with any kind of semantic theory which treats the meanings of sentences as iunctions of component variables. 
4.5 We have so far discussed no consideration which requires us to revise our original proposal (4.1) concerning the relationship between o -characterizations and g-charactizations.

There is, however, reason to believe that vexy many concepts, including everyday physical-object concepts, are indeterminate in the sense covered by Waismann's term 'open texture' and Carnap's 'intensional vagueness' 1 . If this thesis is correct, it must follow that for many expressions in natural languages the enterprise of constructing a complete substantive semantic characterization lacks feqsibility, in the sense that there is no end to the series of conceivable hypotheses which would need testing in order to establish conclusively the sewantic rule governing the informant's interpretation. For any given expression investigated, the main features to be incorporated in the semantic characterization will doubtless become clear soon enough; but anyone who reflects upon the matter can easily coavince himse1: that he would be hard put to it to elaborate the finex details of his own interpretation of words such as house, rogd, chair, glow, latie red, etc. applied to objects and events in his familiar environuent.

1. Waismann 1945, Carnap 1955. 
The problem of 'open texture' and semantic indeterminacy of expressions is dealt with (rather than solved) in the conventional dictionary by the introduction of a more or less arbitraxy linit to the information supplied under each entry. For example, the Shorter Oxford Bnglish Dictionary entry for house tells us that this word means 'a building for human habitation, esp. a dwelling-place'. The same entry also indicates various other meanings for house, but fails to tell us e.g. whether cottages count as houses, whether militaxy barracks count as houses, whethex caravans count as houses, whether igloos count as houses, etc. It does not tell us what to walke of a sentence like phat's not a house: it's a block of ilats, nor of That's not a house; it's a palace.

From the viempoint of semantic theory, it is clearly unsatisjactory to have to concede that this is the best that can be done, ie. that it is arbitraxy at what point we draw the line and say that semantic description is complete. As far as symonymy is concerned, the difficulty is particularly acute in that we have so fax explained semantic equivalence of two expressions as identity of their respective semantic characterizations. But if we are now to concede that the completeness of such a characterization is arbitrarily determined in each case, or that there may be some expressions for 
for which the characterization cannot be completed, then it would seen that, at least in such cases, we have no real account of symonymity at all.

Thus a critic might object : 'It is meaningless to say that $\underline{a}$ and $\underline{b}$ are synonymous unless $\underline{a}$ and $\underline{b}$ are expressions which are semantically determinate. But if they are expressions for which the substantive semantic characterizations are incomplete and cannot be completed, the claim that a and b are synonymous can, in principle, be neither verified nor falsified; For, conceivably, although the incomplete characterizations match so far, they might not match when complete. On the other hand, conceivably, incomplete characterizations which differ might tum out to match when complete. A claim which can be neither verified nor falsified caunot be taken seriously.' Such a critic, in effect, requires a criterion of semantic determinacy as a prexequisite to clains of symonymity.

4.51 One possible way of replying to this cribic would be to involxe what way be called the 'standard meaning' axgument. Whis argument would begin by calling attention to the fact that the alleged semantic indeterminacy of expressions does not often show up in everyday discourse, and suggesting that if this is so it j.s because for wost purposes of everyday discourse the indeterm- 
inacy does not greatly mattex. Doubtless there are cases - issuing guarantees, conforming to legal requirements, giving evidence in a court of law, etc. - when it may emerge that there is uncextainty about the exact meanings of rords; but these instances axe on the whole few and far between, except in such speciálized axeas as philological and philosophical discourse. If that is so - the argument rould run then semantic description need not bothes about indeterminacy any wore than the average language-user does. All that commonsense requires is that we specify for a given expression its 'standard meaning', and leave dispute about marginal cases to those who axe interested in logowachy.

In support of this view, it might perhaps be urged that this is just how, as language-users, we treat questions of meaning. We do not in practice resort to counting raindrops in order to determine whether it is a storm or only a showex. We understand what is meant when someone says There was a storm or There was a shower by refexence to some kind of imaginary 'standard case' of a storm ox a shower. Accordingly, the 'standard meaning' of an expression may be stated simply by giving an account of the relevant 'standard case'. In short, the problem of semantic indeterminacy is created - or at least highly magnified - by the very attempt to introduce irrelevant precision into semantic descriptions. 
This somewhat Platonistic view has a superficial plausibility; but it will not satisfy the 'open texture' critic on various points. For it seems dubious that meaning is normally a mattex of tacit allusion to 'standard cases'. There may perhaps be an imaginary 'standard case' of the cat being on the mat, in the sense that one could describe an example whexe no-one would quibble about whether the cat was on the mat or not, e.g. Tibs curled up asleep right in the middle of the mat. But if $I$ say The oat is on the mat when, perhaps, it has only its two front paws actually resting on the mat, I am not describing some 'degenerate version' of the 'standard case'. If my description is correct, it is because this actual case, like the 'standard case', is a perfectly good instance of a cat's being on a mat.

The 'standard meaning' argument seeus to confuse two quite separate issues. There is the question whether we should all a gree what to say in a particular case, and there is also the question whether a given meaning can properly be defined in terms of approximation to an uncontroversial excmplar. If someone disagrees that it is the right thing to say The cat is on the pat when the cat has only two front paws resting on the mat, on the ground. that this is not suficiently like the standard case of Tibs curled up, then he tacitly assumes that The cat is on the mat is in some sense 
short for a more complete description of the standard case. But this is no way to refute the 'open texture' critic: his point is the difficulty of detexmining exactly what it is everyone is agreed on because of the difficulty of determining what it is anyone is committed to. But insoiar as anyone's comatwent can be determined, then in principle everyone's can be determined。

4.52 The way round this difficulty which it is proposed to take here is to revise the notion that the relational chaxactexization ' $\underline{a}=\underline{b}$ ' stands $f$ or identity of the complete substantive characterizations of a and $\mathrm{b}$ respectively. Instead, it is praposed to treat synonyur as definable by reference to criteria for the formulation of substantive charactexizations, i.e. we talke advantage of the fact that while it may not be possible to specify complete semantic characterizations for a and $b$, nonetheless it may be possible to adopt a particular way of formulating the criteria governing admission or exclusion of itens with respect to substantive semantic characterizations which will enable a couparison relevant for judgments of equivalence to be made.

The proposal i.s to let ' $\mathrm{a}=\mathrm{b}$ ' gtand $\mathrm{for}$ item-by-item matching of substantive characterizations: we opt, in other words, for treating the correctness of ' $\underline{a}=\underline{b}$ ' as depending on 
the same items being admissible to, or excluded frow, the substantive semantic characterizations of a and $\mathrm{b}$.

4.53 Having made this move, we are then at risk in claiming the synonymity of $\mathrm{a}$ and $\underline{\mathrm{b}}$, in the sense that counterevidence may be produced which will reinte the claim (i.e. debar a

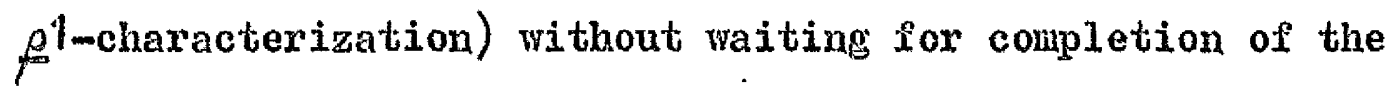
of-characterizations. 1

In oxdex to validate this move, we have now to propose some way of determining whether any given item is to be aduitted to ox excluded from any given semantic characterization. But if any such proposal is not to be vacuous, it wust be integrated in quite specific ways into a theory of linguistic lnowledge.

1. The move is intuitively satisfactory, in that we do not feel that we need to know exactly what two expressions mean in order to be sure, in many cases, that they mean something different. 
5. Synonymy and Iinguiglic knowledge. 
5.0 It would be idje to discuss the role of symonymity statements in grammax and semantics unloss it could be shown that, in principle, it is feasible to formulate procedures which will malre the constzuction of symonymity statements fox I possible. But procedures of this kind cannot be formulated independently of the adoption of sowe specific concept of 'Jinguistic knowledge'; for we cannot, ultimately, say what will count as evidence ${ }^{\prime}$ ox or against a given syanymity statement unless we are cleax about where the bouadaxy between the linguistic and the nonlinguistic lies.

5.01 A concept of linguigtic knowledge may be delinited with sufficient precision for our purposes by specifying (i) how wuch about communication-in- $\mathrm{I}_{\mathrm{H}}$ is to be regaxded as explained by the linguist's descriptions, formal and semantic, of the set of items constituting the linguistic expressions of $\underline{L}$, and (ii) what conditions must be satisfied in respect of a given expression and given item of lnowledge such that the itien of lmowledge counts as linguistic knowledge of that expression. On the foxmal side, relevant proposals have already been put forward in previous chaptexs. It remains to deal with corresponding questions on the semantic side. 
5.1 The most ambitious concept of 'linguigtic knowledge' that could be entertained would, preswubly, be one which made the whole process of successful comunication-in-

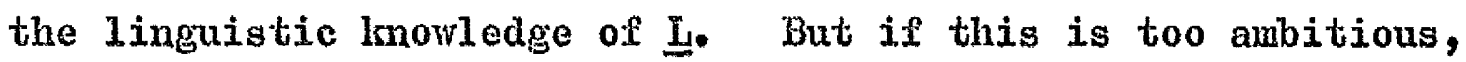
as it certainly seens, the question arises as to where a line shall be drawn between those features of conmunication acts (speech acts, acts of writing) which depend on linguistic lnowledge, and those which involve nonlinguistic judgments of various lriads.

5.11. An important factor in this decision is the evidence from natural languages that the category of a speech act is not uniquely determined by the particular expressions used (as might, conceivably, be the case for some non-natural languages). Since the assigument of the correct speech act category is clearly of prine importance for effective communication between language-users, this suggests an obvious place to fix our boundary, and an obvious way to do it in terms of language description. By excluding any indication of speech act categories ${ }^{1}$ from the characterizations of the sentences of

1. This would be as a general principle; but it is easy to think of certain kinds of expression(e.g. greetings, farewells) where it would be difficult to apply this principle, and absurd to boot. The difficulty in some cases of stating the meanings of expressions independently of descriptions of the specific speech acts with which they are normally associated is well known. (It is forcefully stated in Halinowski 1923.) 
a natural language, we place a significant limitation on the postulated contribution of linguistic lnowledge to speech act interpretation. ${ }^{2}$ 2

2. Other proposals can doubtless be argued for: it is not so far clear that they can be argued for convincingly. Searle suggests that if 'we can reduce all illocutionary acts to some very small number of basic illocutionary types' then we might assign to the deep structure of every sentence 'a simple representation of its illocutionary type'. He rejects, however, the possibility that 'illocutionaxy act rules would attach directly to elements (formatives, morphenes) generated by the syntactic component, except in a few cases such as the imperative' (Searle 1969 \$3.3). Boyd \& Thome want to go further than this, but theix progranme seems to be based on a misunderstanding of Austin's position, e.g. they refer to 'Austin's main point, which is that a complete account of the meaning of a sentence cannot be restricted to semantic analyses as they are usually understood and that they must be extended to include information about the krind of speech act involved in utbering the sentence - that is, its illocutionary force' (Boyd \& Thorne 1969 p.58: but see Austin 1962 p.100), They appear to exvisage an analysis in terms of two basic categories: 'statements and imperatives (the latter being subdivided into commands and demands) are the primary speech acts and all others are in a sense modisications of then. The point is that it seems that the analysis of all performatives can be produced through the addition of other features to the features STIATS and IMP. For example, insigt can be used to make what might be described as a modified statement (I insist that he went), a modified command ( $I$ ingist that you $g_{0}$ ), or a modified deuand ( $I$ insigt that he go). In each case the analysis of the verb insist differs from that of the verb state or the verb command or the verb demand by possessing a feature which-

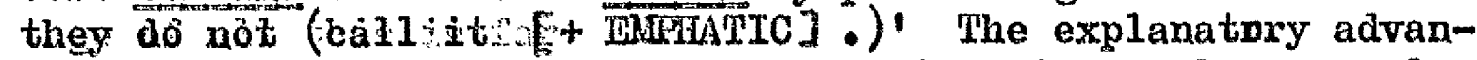
tages of all this is far from clear, since it merely re-analyses what would nomully be regarded (cr. Shorter Oxford Bnglish Dictionary, art, insist) as three different meanings of the verb insigt. Burtherwore, the analysis is highly questionable. (Is the difference between saying I insist that you go and I command you to go a matter of a certain emphas is added to the order? And even if it were, and were only that, 
2. (cont.) can the kind of 'emphasis' involved simply be equated with the kind of 'emphasis' which marke the difference between I insist that he went and I state that he went? This seems to be simply playing with words.) But questionable or not, the new analysis adds nothing to a semantic description of sentences involving insist, since their semantic differences were always recognized anyway (i.e. as different 'meanings' of insist). Nor is this all. Such cases as insist can be subsumed under a general rule (i.e. one applying to various other verbs as well) that utterances of sentences with the verb in the first person singular present indicative can have - but do not invariably have - the appropriate performative force corresponding to their meaning. In other words, we can better explain what kind of speech act is being performed on these occasions by reference to the meaning of the verb involved than by calling it an 'emphatic statement', or an 'emphatic command', etc. (Since Austin clearly realized this, it seems a pity to invoke his authority in support of such a feeble analysis.) Finally, it is doubtful whether Boyd \& Thorne have a firm grasp of the notion of a speech act at all, for they assert that 'under certain circumstances we substitute one kind of speech act for another. For example, under some circumstances we are likely to use the question Will you pass the salt?rather than the command Pass the salt and we would be surprised if someone took it as a question and not a command.' Anyone who can say this must be confused over the difference between semantic categories (e.g. 'Interrogative sentence', 'imperative sentence') and speech act categories (e.g. 'question'). There is no sense in which one kind of speech act is here being substituted for another: on the contrary, Will you pass the salt? and Pass the salt are in normal circumstances both used in speech acts to which one would assign the same category, 'request'. 
5.112 The boundary thus set up coincides with that normally assuned in post-Austinian philosophy of language, e.g. by Strawson:

1 To know the meaning (or meanings) of a sentence of a language is to be at least partially equipped to understand how any serious utterance of it by a particulax speaker in a particulax context is to be taken. But evidently to grasp the whole of what should be talken to be intended to be understood by such an utterance is generally something more than merely to know the meaning of the words uttered. Somewhere in between grasping the formex and lmowing the latter comes the ability to identify any propositions actually expressed in the making of the uttexance. It comes in between the two, on the one hand, because sentencemeaning alone, without help from the context of uttexance, will rarely reveal just at what points the general concepts which figure in a proposition are there conceived as attaching to the world; and, on the other, because lmowledge merely of what proposition is expressed does not include lnowledge of how its expression is to be taken, or of all it is to be taken to imply. If, then, we speals of the whole of what way properly be taken to be intended to be understood by the making of a paxticulax utterance as the force of 
that utterance, we have the trio: force, proposition(s) expressed (if any), sentence-meaning.' 1

It should perhaps be emphasized that pxopositions are not here conceived of a sentence-meanings (as they have sowetines been construed ${ }^{2}$ ). They stand on the irontiers of linguistic knowledge, and their 'Ixontier status' is reilected in their classification into 'synthetic' and 'analytic'. Analytically true propositions constitute the limiting case in which linguistic lnnowledge alone takes us furthest towards comprehending the force of an utterance. To say this is, of course, to leave untouched the task of detexmining which such propositions are, and to this we unst retura below.

5.113 But iirst it should be noted that the proposed semantic boundary for linguistic knowledge coincides reasonably well with intuitive notions of what it is to understand the meaning of a sentence, and with plausible tests for establishing whether or not someone lnows what a sextence means.

For example, let us suppose that there are two readers of Kipling's Plain Tales from the Hills who place different interpretations upon the first sentence 'To the wittiest woman

1. Straws on 1967 pp. 9-10.

2. Staal 1966, Lewmon 1966, Garner 1970. 
in Indxa I dedicate this book', solely in that reader $A$, unlike reader $B$, considers that what Kipling says in that sentience might be true or false. Now we should certainly wish to say that xeader A had iailed to grasp something; but his failure is a failure to grag the force of the speech act ${ }^{l}$ rather than a failure to understand the meaning of the sentence. If there is no respect in which $A^{\prime}$ 's bizarre supposition about the truth-or-falsity of what IKipling says precludes $A^{\prime}$ 's passing all the reasonable comprehension tests which reader $B$ would pass as to the meanings of the words exemplified, and of the exemplified devices for combining them, then the conclusion must be reached that $A$ and $B$ arrive at their differing interpretations on the basis of the same semantic knowledge.

Someone: might, perhaps, chocs e to maintain that one of the key tests is what you would say if asked whether what Kripling here says is true or false, and this is the test A fails to pass. But the motivation for setting up this test is not just weak but thoroughly obscure. For the sentence in question is not one which could never be used to make a trueox-false statement at all (Kipling might conceivably have

1. Cf. Austin 1962 p. 33 et passim. 
uttered it appropriately on some other occasion, e.g. in the course of describing the book to an interviewer on the eve of its publication, to wake such a statement). Reader A's mistake, therefore, seems best described by saying that he here incorrectly assigns the speech act category 'statement', although lnowing perfectly well the meaning of the sentence in question.

Assigning the correct dategory to a speech act is characteristically unlike assiguing the correct meaning to a sentence in that there is no total to be axrived at which is the product of component parts. The judgment relates to a unit which is the whole utterance, and any mistake is a mistake about the category of the whole utterance. One wight be wrong about the meaning of one particular word in a sentence, although right about the rest. Such a mistake has no analogue in judging the category of a speech act. For we do not (to adapt a rewaxk of L.J. Cohen ${ }^{2}$ ) uttex one word of statement and six of something else: it is the whole utterance which constitutes the statement, not any part of it. Whereas it is typically one word or group of words which constitutes e.g. the referring expression in a sentence.

2. Cohen 1962 p. 45. 
Failure to pay attention to such distinctions is a potbatial source of confusion in semantics. It may result (as with Katz \& Fodor's explanation of 'paraphrase' already conuanted on, 4.12-4.13) in a misleading use of verbs such as say, mean, applied indiscriminately both to speech acts and to expressions uttered in speech acts, or (as with lyons's theory of 'context-bound synonymy', 4.32-4.321) in a lack of clarity concexning the role of context in determiningintexpretation. It may also lead to confusion over such a notion as 'semantic anomaly', which plays an important part in the semantic theory of Katz \& Fodor and in the discussion of analyticity generally. For example, somono might be led to consider as semantically anomalous a sentence like - to take Austin's example ${ }^{3}-$ I appoint this horse copsul, where the oddity is due not to the fact that the sentence lacks a plausible 'xeading' 4 , but to the fact that the corresponding speech act lacks force.

Such considerations argue in favour of the proposal to let the $g$-characterization of an expression a represent the lnowledge a competent spealsex of $\lfloor$ may draw upon in interpreting

3. Austin 1962 p. 35 .

4. On the contrary, the sentence is semantically quite appropriate for the conmunicational purpose envisaged, if only there existed a social procedure which gave its utterance the required iorce. 
any speech act, just insofar as that intexpretation is detiermined by the use of a in the speech act in question. Thus a of-charactexization is not, as such, called upon to represent the knowledge which enables the hearer to assign the correct speech act category to an utterance.

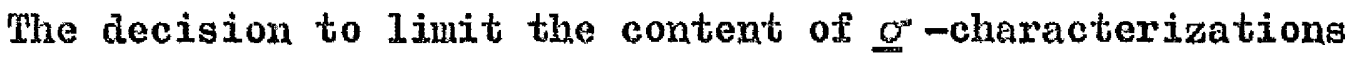
accordingly may, in the case of some non-natural languages, be otiose (e.g. for languages where all speech acts belong to the same category). Nonetheless, as regards natural languages a distinction is warranted by the radical difference in the kind of judgment called for.

On this view, linguistic knowledge does not explain e.g. understanding the difference between Go for a walk as a suggestion and Go for a walk as an order, nor the difference between you are asling for trouble as a warning and You are asking for trouble as a threat, unless such differences axe regularly signalled by linguistic means, e.g. by systematic differences of intonation ${ }^{5}$. In such pairs the force of the speech act may be different, while the meaning of the expressions is the same.

5. There will doublles be horderline cases - these may be such - where it is not clear whether we should say there is a difference of meaning. 
The distinction is of particular inportance as regards synonyuy, since it may be perfectly possible to find sentences which are nomally used with the same force in speech acts but have difierent meanings (perhaps Would you mind shutting the door? and Shut the doox if you please ${ }^{6}$ ), and also sentences which are normally used with diffexent force in speech acts, but have the same meaning (perhaps Primatology is the sdudy of primates and Primatolog is primatology 7 ).

5.114 In acceptance of the foregoing there is an implicit rejection of 'neo-Austinian' definitions of meanings in terms of speech acts, e.g. the definition of sentence meaning proposed by Aziston:

6. Ividence of a difference of meaning is the fact that $I$ may say Yes to the first if $I$ am objecting, whereas if $I$ say Yes to the second $I$ am signifying readiness to comply. (Some-one may say Yeg to the interrogative sentence to indicate compliance; but if he does, this shows he is taking the utterance of that sentence as having the iorce of Shut the doox if you please, or something similar, i.e. his Yes is not, as in the other case, the answer to a question.)

7. The first is normally, we may assume, intended to have the force of a definition, which it is difficult to attribute to the second. 


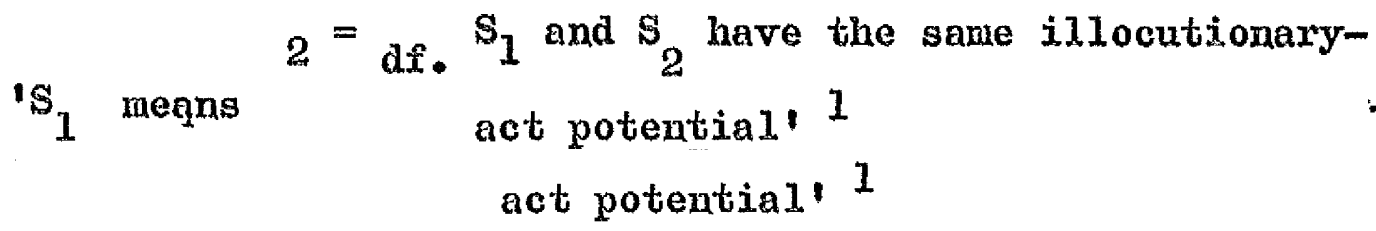

and associated accounts of synonyuy, e.g.

$$
\begin{aligned}
W_{1} \text { means } W_{2}=d f . & W_{1} \text { and } W_{2} \text { can be subgtituted for each } \\
& \text { other in a wide range of sentences } \\
& \text { without altering the illocutionaxy act } \\
& \text { potentials of those sentences' } 2 .
\end{aligned}
$$

The reason why semantic definitions of this lsind do not provide an acceptable basis for an account of synonymy in natural languages is quite simply that differences in illocutionary act potential can sometimes be due to a formal difierence between expressions. To talre up, by way of illustration, the example cited in 5.113 , it is clear that there will be illocutionary acts which can be performed by uttering Primatology is the study of primaties but which cannot be performed by utteriag Pximatology is primatology, e.g. the illocutionary act of getting $X$ to understand) what prinatology is (granted that $X$ does not know what primatology is: fox exauple, supposing he has asked the question 'Can you explain to no what prinatology is?'). For to say to someone, in effect, 'I gather you do not understand what prinatology is, but, since you ask we, I will enlighten you

1. Alston $1964 \mathrm{p} .36$.

2. Alston 1964 p. 37 . 
by telling you that primatology is primatology' would be sonewhat like saying 'I know the doox is shut, but shut it all the same'. In these and similar cases the speaker would be failing to 'tale responsibility' (to use Alston's phrase ${ }^{3}$ ) for his speech act in the appropriate way.

What is missed in the 'illocutionary act potential' definition is precisely the sense of 'meaning' in which the symonymity of $\underline{a}$ and $\underline{b}$ accounts for the correctness of the explanation 'a is b'.

5.2 If we accept that a sewantic description of $\underline{L}$ must be based on a priox decigion to differentiate item of lmowledge into two classes (those which count, and those which do not count, as items of semantic knowledge ajout particular expressions), and $i f$ the substantive semantic characterizati ons contained in a semantic description of $\mathrm{L}$ are interpreted as statements to the effect is (the speaker of 2 ) knows that .....', then a method of formulating the comtent of semantic charactexizations involves a procedure for selecting certain items from the totality of lnowledge attributable to the

3. Alstion 1964 p. 43. 
speaker. Such a view incurs the obligation to demonstrate that non-question-begging tests for classifying items of lnowledge in the mannex required are, in principle, available.

To talre a simple case, if we treat the semantic charactexization

'dibatag: long-necked antelope.....' as representing the proposition:

is lnnows that long-necked antelopes.....are called dibatags ' the formulation of the semantic characterization implies a decision to include certain items of lnowledge relating to a cextain class of animals as part of the spealse $x^{\prime}$ s linguigtic knowledge, and to exclude certain others. Thus fox any item of knowledge about dibatags attributable to the spealser who knows how to use the word djbatag (e.g. that they have an average gestation pexiod of $\underline{n}$ days, ox that they are alwost extinct) the question arises whether it forms paxt of the speakex's linguistic knowledge of the expression dibatag. This question is, in effect, the question of delimiting what Katz \& Fodor call the 'upper bound of a semantic theory'. 
Its relevance to analyticity is that according as different items are included in ox excluded fxom a semantic charactexization, so different seutences will consequentially be exhibited as analytic.

Katz \& Fodor claim:

'Since a complete theory of setting selection must represent as part of the setting of an utterance any and every feature of the world which speakers need to determine the prefermed reading of that uttexance and since...practically any item of information about the roxld is essential to some disambiguations' it follows that 'such a theoxy cannot in principle distinguish between a speaker's lmowledge of his language and his lkaowledge of the world because, according to such a theory, part of the characterization of a linguistic ability is a representation of vixtually all lnowledge about the woxld speakexs share.' 1

As an example of a piece of nonlinguis tic infoxmation Katz \& Fodox cite the fact that lions, but not children nox buses, axe often kept in cages. Innowledge of this fact, they argue

1. Thatz \& Fodox 1963 p. 489. 
would be required for the selection of correct readings for the following three sentences:

Should we talke the lion back to the zoo?

Should we take jumiox back to the zoo ?

Should we take the bus back to the zoo?

Thus, even if informants concurred in assigning no more than one reading to each of the above sentences in isolation, a semantic description of Inglish should nonetheless - on this view - waxk each sentence as ambiguous, sänce the informanta? interpretation is here based upon an item of information whiok happens to be common lnowledge, but not linguistic lnowledge. In the example cited, it is clear that the decision to maxk each sentence as ambiguous means excluding certain information from the semantic characterizations of various words, $e . g$. we exclude from the semantic characterization of the word lion any such metalinguistic description as '... often kept in cages...'. But the question arises: what are the grounds for this exclusion? To axgue that the exclusion is justified because the lknowledge that lions axe often kept in cages is nomlinguistic knowledge would be beside the point; since for any given item of nonlinguistic knowledge we can easily fommlate a corresponding ithen of information about 
the use of a linguistic expression, e.g. that lion denotes a class of aximals.... often kept in cages. The question that needs to be answered is why this does not count as part of the speaker's semantic lnowledge of the woxd lion.

The same issue is rajsed in a more general form by Bolingex's quexy: 'where do semantic maxkers come from?to Bolinger points out that Katz \& Fodor allow that in communication situations there are occasions where 'we achieve a disambiguation by way oi something that is not a semantic maxisex', and asks:

tBut why is it not a semantic maxkex? Where do markers like (Animal), (Physical Object), (Xoung), and (Female) come fxom if not fxom oux knowledge of the world? What is strange about (Shoeweaxing) as a semantic markex - not as general, surely, as (Tewale), but general enough? The discalced branch of Carmelite monks is identified by $i t$, and it crops up every now and then as a mark of status....' 2

To such questions, however, neithex Katz \& Fodor nor Bolingex axe able to supply an answer. Katz o fockor take for granted a digtinction between semantic and nonlinguistic knowledge, but attempt no explication of the distinction. At leagt two reasons requixe that such an attempt be made. First, until it has, the upper bound of a semantic theory remains unitixed, 
i.e. we have no assuramce that literally any fact at all aboutb lions (e.g. that Nero kept them as pets) might not turn out to be part of the meaning of the word lion. Second, as long as the upper bound of a semantic theory remains unfixed, the problem of analyticity remains unsolved, for we shall be unable to say with cextainty which the analytic sentences of 悬 axe. As Staal obsexves in connexion with another example (Whales are manmals), 'the semantic theory of a language does not solve this problem, but presupposes its solution. For the answer would depend on the way semantic trees axe constructed. For the above illustration, [mamal] nay ox may not be considered a semantic element of the tree for whale, dependent on whethex this zoological information is ox is not considexed paxt of the native speakex's competence' 3 .

3. Staal 1966 p. 79. Staal, however, wants to have it all ways at once; fox he cladins that Katz (Katiz 1964) showed how ta semantic theory enables us to decide whjch sentences of a natural language axe analytic. The decision can be obtained. quite mechanically with the help of formal definitions 20 r analyticity, contradiction, etc. Hexe no cixcularity is involved, since the construction of a semantic theory does not depend on notions like analyticity...' (Staal 1966 p.68). The claim that no circularity is involved is repeated (Staal 1966 p.72). But nothing could be wore patently circular; for the construction of a semantic theory depends on deciding what to count as included in the meanings of particular words, and since the type of semantic theory advocated by thatz \& Fodor has as one of its objectives to explicate the semantic conbribution of individual words to the sentences in which they occur, that in turn presupposes that a decision has already been taken as to whethex eog. the fact that whales are mammals counts as semantic knowledge. If it does, Whales are mammals will turu out to be analytic. 
5.21 The solution which seems best to meet the requixemexts of empixical linguistic analysis is to count lnowing the meaning of an expression as including lonoring that $p$ if and only (i) all speakers of $i$ know that $\mathrm{g}$, and (i.i) the assumption that all speakers of $\mathrm{I}$ know that $\mathrm{g}$ is required to explain their normal interpretation of some sentence ox sentences of $\underline{I}$ comprising or containing the expression in question.

Where $\mathrm{p}$ is an item of lrmowledge relating to the use of an expression a, we can repregent $p$ by including an appropriate metalinguistic expression $\pi$ in the semantic characterization of a:

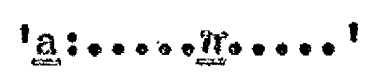

Then the following rule (R) will govern the forumlation of the content of substantive semantic characterizations:

(R) : Trox any expression a, the charqctexization includes a metalinguistic description $\pi$ if and only if (i) the information represented by $\pi$ is known to all speakers of $\mathrm{I}$, and (ii) the information represented by $\pi$ is utilized in the intexpretation of some sentence of $\underline{\mathrm{L}} \cdot$ ' Thus under $\mathbb{B}$, if $\mathrm{a}$ is the expression 1 ion and $\pi$ is the metalinguistic expression '...often liept in cages...', gor inclusion of this expression in the characterization of a $i t$ needs to be shown (a) that all speakers of L know that lion 
is a word for a lrind of cxeature often kept in cages, and (b) that this lnowledge is utilized by all spealrexs of $\mathrm{L}$ in their interpretation of some sentence of L.

Testing for (a) is, we may take it, straightforward enough. Testing $f(x)$ (b) can be undertaken by constructing sentences about lions in which some point of interpretation (e.g. a disambiguation of pronominal reference) is dependent on lnowledge of the fact that liom denotes a kind of cxeature often lrept in cages, and investigating whether spealsers of I utilize this knowledge in interpreting the sentences ${ }^{1}$.

This proposal does not incux the objection roiced by Katz \& Fodor againgt a theory of setting selection, that it involves as 'paxt of the characterization of a linguistic ability...a representation of vixtually all knowledge about the world spealsexs share'. This objection is met by the

1. Katz \& Rodox's exayple is somewhat more complex than this in that the simultameous utilization of several items of knowledge is involved, namely: 'lion:.... of ten kept in cages....', 'bus :.......not often kept in cages.... $\{$, 'child:.... not often kept in cages...'. But we can run tests for the simultaneous utilization of such knowledge with sentences such as John could see buses and children and lions in their cages, and determine whether thejx cages is interpreted as refexring to the lions' cages, or, as is syatactically possible, to the cages of the buses and children as well. It would be essential to construct the test in such a way that no information relevant to the disambiguation (e.g. that the lions were in cages, but not the buses nox the children) was supplied by the context. 
satisfaction of both conditions incorporated in $\underline{\mathrm{R}}$, i.e. it is not sufficient that an item of knowledge should be shared by all speakers of $\mathbb{L}$, but it unst be shown also that this item of lnowledge is utilized by all speakers in their interpretation of cextain sentences of $\underline{L}$. It is this latter condition which provided the justification for including the relevant inforwation in the characterization of a linguistic ability. It might pexhaps be the case in some particular instance that all items of knowledge common to speakers of $\underline{\mathrm{L}}$ could be shown to be utilized by the spealkers of $\mathrm{L}$ in their interpretation of sentences. But if that were so it would be an empixical fact about that linguistic community, not a consequence which must follow from the adoption of $\mathrm{A}$ in the linguistic analysis of $\mathrm{L}$. We may conclude, then, that the application of $\mathrm{n}$ satisfactorily determines the upper bound of a semantic theory of $I_{\text {. }}$

5.22 On the basis of $\mathrm{R}$, we may define synthetic synonyuyin-L by (i) making it both a necessary and a sufficient condition for relational charactexizations of type $\frac{\text { Spl that there }}{f}$ be no metalinguistic description $\tilde{\lambda}^{\prime}$ such that $\underline{\eta}$ is excluded Irom the substantive semantic characterization of one but not the other of the two I-expressions in question, and (ii) making it both a necessary and a sufficient condition for relational 
characterizations of type $\$ g e$ that there be a certain metalinguistic description 2 such that $\underset{f}{T}$ is excluded from the substantive semantic characterization of one L-expression but not the other.

Similarly fox analy'́ic synonymy-in-L chaxactexizations of type $\frac{A g l}{7}$ and $A g ?$ will involve the same guarantees for matching pairs of meaningful elements which occur as components of two syathetically symonymous expressions.

The angwer thus proposed to Quine's problem of distinguishing synonyms from expressions which mexely agree extensionally runs in brief as follows. fwo co-extensional expressions (é.g. cxeature with a heart and creature with kidneys)will be counted (synthetica11y) synonymous if all spealrers of $\mathrm{L}$ know that they are comextensional, and utilize this lmowledge in intexpreting appropriate sentences containing these expressions. They will not, however, oout as analytically synonymous expressions if there axe semantic diffexences between coxresponding pairs of components (e.g. a heart and kidneys). For such pairs of expressions as afford no basis for the semantic comparison of component partis (e.g. pomelo and shaddock) a distinction between synthetic and analytic synonymity cannot be drawn. We need not be moved by objections tothe effect that this solution simply elevates factual lnowledge to the status of semantic lnowledge on condition that it play a role in interpretation. For we may reasonably, at this point, inquixe of our 
objector what better reason he can think of for calling knowledge 'semantic'.

Accordingly, any truth expressed in a sentence of the form 'All and only as are bs' will be analytic on condition that substantive semantic characterizations for a and $\mathrm{b}$ admit and exclude the same items. This can be tested empirically, since the proposals adrocated above allow us to determine, fox any item of lonowledge whatsoever, whether it shall be represented in the semantic chaxactexization of any given expression.

Norwally, it will not be difficult to construct fox a and $\underset{n}{b}$ exactly parallel tests to decide whether a particulax item shall be included in or excluded from the substantive semantic characterization. For example, we might wish to test the hypothesis that the word bachelor is interpreted as applying to a man who has no wife. If that hypothesis is coxrect, we should expect informants to be able to identify the subject of ras reiused admission in Peter arrived at the club with John, who also brought Peter's wife in the car, but, being a bachelor, was refused admission; to detect an anomaly in There were twenty trade union of ficials at the meeting, of whom ten were bachelors and at least twelve had brought theix wives; etc. Let us suppose that the results with these tests are positive, i.e. confixm the hypothesis. Now tests can be run with the same sentences, substituting unmarxied map and 
unnarried men for bachelor and bachelors respectively. If these tests give the same results, we conclude that both for bachelor and for unwarxied man the substantive semantic characterization nust be so formulated as to include the information that the expression is interpreted as applying to a man who has no wife.

When we can elicit no furthex items of knowledge which appear to determine how informants intexpret sentexces containing a and sentences containing $\underline{\mathbf{b}}$, and any hypothesis confirmed (ox rejected) for a has been coxrespondingly conPirmed (or rejected) for $\underline{b}$, we may conclude that $\mathrm{a}$ and $\mathrm{b}$ are (syathetically) synomymous. They will also be analytical ly synonmyous to the extent that similar results can be obtained for matching pairs of meaningful components.

5.23 The proposed method allows us to take account of other than 'cognitive' differences between expressions. It allows for as many different dimensions of semantic variation as we can establish to be empirically relevant to conmunication-in-l. For example, if it is established that all spealrers of $\underline{L}$ lmow that nigger is a more derogatory tem than negro and use this knowledge in assigning an interpretation to some sentence(s) of $\underline{L}$, the respective semantic characterizations for negro and niggex must reflect this difference. 1 
5.3 In conclusion, it may be claimed that an account of synomynuy based on $\mathrm{R}$ will be adequate for purposes of the linguistic analysis of natural languages in just this sense: that it provides an unobjectionable basis ior explaining what it means to incorporate into a description of L a statement to the effect that two L-expressions have, or do not have, the same meaning, and renders any such statement amenable to vexification.

Such an account, howevex, counits us to a concept of linguistic knowledge which goes considerably beyond the notion of the 'internalized rules' of the ideal spealser-hearer of Lي. This ifignent of modern linguistic theory is the fashionable myth who has replaced Saussure's mumbo-jumbo about a language existing only in the collective mind of the community. But, although ideal, he has his shortconings; and one of them is that the question of what counts as linguistic lmowledge for him not only cannot be answered, but cannot even be raised. 
Bibliography

This list includes all itens to which reference is made in the present study, and others dixectly relevant to the topics discussed.

Abraham \& Kiefer 1966

5. Ab́raham \& $\mathrm{F}$. IKiefex A theory of structural semantics, The Hague/Paxis, 1966.

Alston 1964.

W.P. Alston Philosoply of Language, Englewood Cliffs, 1964.

Austin 1962

J. l. Austin How to do things with words, ed. J.0. Urms on, Oxford, 1962.

Bach 1964

W. Bach An Introduction to Transformational Grammars, New York, 1964.

Bax-Hillel 1967

Y. Bar-Hillel, review of the Structure of Language, ed. Fodor \& Tatz, Englewood Cliffs, 1964, in Language XIIII, 1967, pp.526-550.

Bazel1 1954 C.F. Bazell 'The sememe', Litexa, Vol.1, 1951, pp.17-31.

Beard 1965

R.W. Beard 'Synouyny and oblique contexts' Analysis Vol.26, 1965, pp.1-5.

Bemnett 1968

D. C. Bennett linglish prepositions: a stratificational approach', Journe1 of Linguistics Vol.4, 1968,pp. $153-172$.

Bloch 1948

B. Bloch 'A set of postulates for phonemic analysis', Language XXIV, 1948, pp.3-46.

Bloch \& Trager 1942

B. Bloch \& G.L.Trager Outline of Linguigtic Analysis, Baltimore, 1942 . 
Bloomfield 1935

L. Bloomfield Ianguage, Iondon, 1935.

Bolinger 1965

D. Bolinger 'The atomization of meaning', Language KLI, 1965, pp. 555-573.

Boyd \& Thoxne 1969

J. Boyd \& J.P. Thome 'The semantics of modal verbs', Journal of Linguistics Vol.5, 1969, pp. 57-74.

Carnap 1947

n. Caxnap heaning and Necessity, Chicago, 1947 .

Caxnap 1955

R. Carnap 'Meaning and synonyuy in natural languages', Philosophical Studies Vol. 7, 1955, pp.33-47.

Catford 3965

J.C. Catford A Iinguistic Theory of translation, 0xiord, 1965.

Chomsky 1955

A. No Chomsly 'Semantic considerations in granmar', Monogxaph Sexies on Language and Linguigtics No.8, 1955 , pp. $141-158$.

Chomsky 195 ?

N. Chomsly Syntactic Structures, 's-Gravenhage, 1957.

Chomsky 1962

N. Chomslry 'The logical basis of linguigtic theory', preprints of papers for the Ninth Intexuational Congress of Linguists, 1962 .

Chuxch 1954

A. Chuxch 'Intensional isomorphism and identity of belie', Ehilosophical Studies Vol.5, 1954,pp.65-73.

Cohen 1962

I.J.Cohen The Diversity of Meaning, London, 1962.

Collins on 1939

W. D. Collinson 'Comparative Synonymics' 'Transs actions of the Philological Society, 1939,pg.5l-77.

Dixon 1963

R.H.W.Dixon Lingujgtic science and logic, The Hague, 1963. 
Ducháčelk 1964

0. Ducháček 'Différents types de synonymes', Oxbis XIII No.1, 1964, pp. 35-49.

Eheling 1960

C.L. Theling Linguistic Units, 'g-Gravenhage, 1960 .

Pillmoxe 1968

C.J.Fillmore 'The case fox case' in Unirergals of

Linguistic Dheory, ed. Bach \& Harms, New York 1968 .

Tischen-Jórgensen 1956

W. \$ischero-Jdrgensen 'The comutation test and its application to phonemic analysis', For Roman Jakobson, The Hague, 1956,pp. 140-151.

Frege 1892

$G_{\text {A Rege }}$ "Uner Sinn und Bedeutung', Zeitschrift: für Philosphie und philosphische Kritils, Vol.100, $1892, \mathrm{pp} \cdot 25-50$.

Garner 1.970

R.T. Garner 'Lemnon on sentences, statements and pxopositions', Analysis Vo1.30, 1970, pp.83-91.

Gleason 1966

If.A. Gleason An Introduction to Descriptive

Linguistics, revised ed., New York, 1966.

Gooduen 1949

N. Goodman 'On likreness of meaning', Analysis

Vol.10, 194.9, pp.1-7.

Goodman 1953

N. Goodman 'On some differences about meaning', Analygis Vol.13, 1953, pp.90-96.

Haxris 1951

7.S. Farxis Methods jin Structural Linguistics

Chicago, 1951 .

Harris 1954

Z.S. Haxxis 'Distributional Structure', Woxd Vo1.10, 1954, pp. 146-162.

Haxris 1967

R. Iarris 'Sewantics and translation', Proceedings of the Tenth International Congress of Linguists, Bucharest, 1967, Vol.II pp. 463-469. 
Haxix is 1970

R. Harris 'Deviance and Citation', Journal of Linguisties Vol. 6, 1970.

Hockett 1958

C.N. Hoclsett A couxse in modern linguistics, New Xork, 1958.

Jones 1962

D. Jones The Phonejue: its Nature and Use, 2nd ed., Cambridge, 1962.

IKatz 1964

J.J. Katz 'Analyticity and contradiction in natural language' in The Structure of Ianguage, ed. Fodox \& Katz, Lnglewood Clifis, 1964, pp.519-543.

Katz 1967a

J.J. Katz 'Recent issues in semantic theory', Foundations of Language Vol. IIT, 1967, pp.124-194.

Katz $1967 \mathrm{~b}$

J.J. Katz 'Some rematss on Quine on analyticity', The Journal of Philosophy LXIV, 1967, pp.36-54.

Katz \& Fodor 1963

J.J.Tatz \& J.A.Fodox the structure of a semantic theory', Iranguage XXXX, 1963. (Page references are to the reprinting in The Structure of Language, ed. Fod or \& Katz, linglewood cliffs, 1964, pp.479-518.)

Katz \& Postal 1964

J.J. Katz \& P.M. Dostal An Integrated Theory of Linguistic Descriptions, Cambridge, Mass., 1964.

Katz \& Martin 1967

J.J. Katz \& T. Martin Ji'. 'The synonymy of actives and passives', the Philosphical Rleview IXXVI, 1967, pp.476-491.

Kiefer 1966

F. Uiefer 'Some semantic relations in a natural language', Foundations of Language Vol. 2, 1966, pp.228-240.

Laksoff 1969

R. Lakroff 'Some reasons why there can't be any some-any rule', Language XIV, 1969, pp.608-615. 
Lemnon 1.966

E.J. Lemmon 'Sentences, statements and propositions', in British Analytical Philosophy, ed. Williams \& Montefiore, London, 1966, pp.37-107.

Lewis 1944

C.I.Lewis 'The modes of meaning', Thilosophy and Phenomenological Research IV, 1944, pp.236-249.

Lijusky 1967

L. Linslky 'Synonymity', Dncyclopedia of Philosophy, Vol.8, 1967, pp.54-5\%.

Iyons 1963

J. Lyons Structural Semantics, 0xiord, 1963.

Jyyous 1968

J. Lyons Introduction to theoxetical Linguistics, Cambridge, 1968.

Nalinowski 1923

B. Malinowski 'The problem of meaning in pximitive languages', in C.K.ogden \& I.A.Richards The Meaning of Meaning, London 1923, Supplement I.

Mates 1950

B. Mates 'Synonymity', University of Califoxnia Eublications in Philosophy XXV, 1950,pp.201-226.

Meckler 1954

L. Weckler' 'On Goodman's refutation of symonymy', Analysis Vol.14, 1954, pp.68-78.

Medlin \& Smart 1957

B.H. Medlin \& J.J.C. Smaxt 'Moore's paradox: synonymous expxessions and defining', Analysis vol.17, 1957, pp.125-134.

Moore 1970

T. Moore 'Synonymy and case graumar', paper read to the Linguistics Association of Great Britain, April, 1970.

Naess 1953

A. Naess Interpretation and Preciseness, 0s1o, 1953. 
Naess 1957

A. Naess 'Synonymity as revealed by intuition', The Philosophical Reviev TKVI, 1957, pp.87-93.

Nida 1949

I. A. Nida Moxphology, and ed., Ana Axbox, 1949.

Olds 1956

M. E. 0lds 'Gynomynity: extensional isomorphism', Mind LXV, 1956, pp.473-488.

Osgood, Suci \& Tannenbawn

C.X. Osgood, G.J. Suci \& P.X. Tannenbawil The heasurement of Meaning, Urbana, $195 \%$.

$\operatorname{Pap} 1955$

A. Rap 'Belief, synonynity and analysis', Philosophical Studies Vo1. 6, 1955, pp.11-15.

Pap 1958

A. Jap Semantics and Necessary Truth, New Haven, 1958.

Pexkins \& Singex 1951

H. Perkins \& I. Singer 'Analydicity', the Journal of Philosophy XIVIII, 1951, pp. $485-497$.

Pike 1947

I. L. Pilse 'Gramatical prerequisites to phonemic analysis', Houd Vol.3 No.3, 1947, pp.155-172.

Pilse 1967

IK. I. Pike Language in relation to a unified theory of human behavior, and xevised ed., The Hague, 1967.

Pxice 1950

R. A. Price 'A note on likeness of meaning', Analysis Vo.1.11, 1950, pp.18-1.9.

Futnam 1954

H. Putnam 'Synonymity and the analysis of belief sentences', Analysis Vol.14, 1954, pp.485-497.

Quine 1943

W.V.0. Quine 'Notes on Existence and Necessity', The Journal of Philosopliy, Vol. 40, 1943, pp.113-127. 
Quine 1953

W.V.0. Quine 'The problem of meaning in linguistics' in $V_{0} V_{0} 0$ o guine Brom a logical point of view, 2nd.ed., Cambridge, hass $;, 1961$.

Quine 1960

W.V.0。 Quine Word and object, Cambridge, Mass., 2960 。

Quine 1961

W.V.0. Quine 'Mwo dogmas of empiricism' in W.V.O.

quine From a logical point of view, 2nd ed., Cambridge,

Quine 1967 Mass., 1961 .

W.V.0. Quine 'On a suggestion of Katz', The Journal of Philosophy, IXXV, 1967, pp.52-54.

Reichenbach 1947

H. Reichenbach yiements of Symbolic Logic, New York, 1947.

Robbins 1952

B. Nobbias 10n synonymy of word-events', Analysig Vol.12, 1952, pp.98-100.

Rolling 1952

C.D. Rollins 'Sameness of meaning - a reply to lin . Wiempahl and others', Analysis Vol.13, 1952,pp.46-4.8.

Rosexberg 1967

J.F. Rosenberg 'Synonyay and the epistemology of linguistics', Inquiry Vol. 10, 1967, pp.405-420.

Rosetti 1963

A. Rosetti. 'Son-type et phonème' Linguisties Vol.l, 1963, pp. 58-59.

lludnex 1950

R. Budnex (A note on likeness of meaning', Analysig Vol.10, 1950, pp.115-118.

Schefflex 1955.

I. Schefflex 'On synonyuy and indirect discourse', Philosophy of Science Vol.22. 1955, pp.39-44.

Schwayder 1954

D. Schwayder 'Some remarlss on synonymity and the language of semanticists', Philosophical studies Vol.5, 1954, gp.145. 
Searle 1958

J.R. Searle 'Broper names', Hind LXVIT, 1958, pp. 166-173.

Searle 1.969

J.n. Seaxle Speech Acts, Cambridge, 1969.

Sellars 1955

W. Sellars 'Putnam on synonymity and belief', Analysis Vo1.15, 1955, pp.117-120.

Sorensea 1963

H.S. Sorensen the Meaning of Proper Names,

Copehhagen, 1963.

Southworth 1967

T. C. Southworth 'A model of semantic structure',

Language XIIII, 1967, pp.342-361.

Spaxcls Jones 1964

K. Sparck Jones Synonyuy and Semantic Classification, Cambridge, 1964.

S.taal 1966

J. I. Staal 'Analyticity', Foundationg of Language Vol.2, , 1966, pp.67-93.

Stati 1966

5. Stati 'Homonymie, synonymie et équivalence en syntaxe', Pevue roumaine de linguistique XI. 1966, pp.133-146.

Strawson 1967

P.F. Strawson Philosophical Logic, ed. Strarson, Oxford, 1967, 'Introduction' pp.1-16.

Straws on 1970

P.I. Straws on Meaning and Truth, 0xford, 1970.

Sweigart 1958

J.W. Sweigart Jx., 'On sameness of meaning' Elilosophical Studies Vol. 9, 1958,pp.38-41.

Thoms on 1952

J.F. Thoms on 'Some remarks on synonymy', Analysis Vol. 12, 1952, pp.73--76. 
Trubetzlkoy 1939

N. S. Trubetzkoy Grundzüge dex phonologie, Rrague, 1939. (Page references are to the 4th ed., Göttingen, 1967.)

Tugendhat 1970

T. Tugendhat 'The meaning of 'Bedeutung' in Frege', Analysis Vol.30,1970, pp.177-189.

Twadde11 1935

W.F. Traddell on defining the phoneme, Language Monograph XVI, BaItimore, 1935.

U1lmann 1959

S. Ul Imann the Princioles of Semantics, 2nd ed., Oxford, 1959.

Waismann 1945

TT. Waiswann 'Verifiability', Proceedings of the Aristotelian Society Supp.Vol.19, 1945, pp.179-150.

Weinrejch 1963

U. Weinreich $10 \mathrm{n}$ the semantic structure of language', Universals of Language ed. J.II. Greenberg, Cambridge, Hass., 1963.

Weinxejch 1966

U. Weinreich 'Ixplorations in semantic theory' in Current Trends in Linguistics, ed. Sebeok, Vol.III, The Hague, 1966, pp.395-4.77.

White 1958

A. R. White 'Synonymous Expressions', The Philosophical Quarterly Vol.8, 1958, pp.1.93-20\%.

Wienpahl 1951

P. Wienpahl 'Moxe about the denial of sameness of meaning', Analysig Vol. $12,1951$.

Winter 1964

W. Winter 'Form and meaning in morphological analysis' Linguistics Vol.3, 1964, pp.5-18.

Wittgenstein 1958

J. Wittgenstein Ihilosophische Untersuchungen, 2nd ed., tr. G.t.M. Anscombe, Oxford, 1958. 
Zife 1966

P. Ziff "The nonsynomyny of active and passive sentences', The Mhilosophical Reviev LXXV, 1966,
pp.226-232. 